\title{
On the implementation and usage of SDPT3 - a MATLAB software package for semidefinite-quadratic-linear programming, version 4.0
}

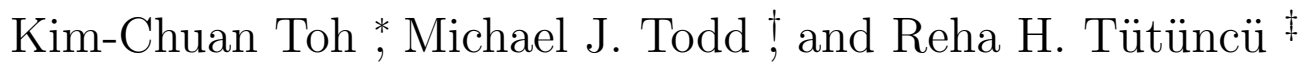 \\ Draft, 16 June 2010
}

\begin{abstract}
This software is designed to solve primal and dual semidefinite-quadratic-linear conic programming problems (known as SQLP problems) whose constraint cone is a product of semidefinite cones, second-order cones, nonnegative orthants and Euclidean spaces, and whose objective function is the sum of linear functions and logbarrier terms associated with the constraint cones. This includes the special case of determinant maximization problems with linear matrix inequalities. It employs an infeasible primal-dual predictor-corrector path-following method, with either the HKM or the NT search direction. The basic code is written in MATLAB, but key subroutines in $\mathrm{C}$ are incorporated via Mex files. Routines are provided to read in problems in either SDPA or SeDuMi format. Sparsity and block diagonal structure are exploited. We also exploit low-rank structures in the constraint matrices associated with the semidefinite blocks if such structures are explicitly given. To help the users in using our software, we also include some examples to illustrate the coding of problem data for our solver. Various techniques to improve the efficiency and robustness of the main solver are incorporated. For example, step-lengths associated with semidefinite cones are calculated via the Lanczos method. The current version also implements algorithms for solving a 3-parameter homogeneous self-dual model of the primal and dual SQLP problems. Routines are also provided to determine whether
\end{abstract}

*Department of Mathematics, National University of Singapore, Blk S17, 10 Lower Kent Ridge Road, Singapore 119076 (mattohkc@nus.edu.sg); and Singapore-MIT Alliance, E4-04-10, 4 Engineering Drive 3, Singapore 117576 .

†School of Operations Research and Information Engineering, Cornell University, Ithaca, New York 14853, USA (miketodd@cs.cornell.edu). Research partially supported by ONR through grant N0001408-1-0036.

${ }^{\ddagger}$ Quantitative Investment Strategies, Goldman Sachs Asset Management, New York, NY 10282, USA (reha.tutuncu@gs.com). 
the primal and dual feasible regions of a given SQLP have empty interiors. Numerical experiments show that this general-purpose code can solve more than $80 \%$ of a total of about 430 test problems to an accuracy of at least $10^{-6}$ in relative duality gap and infeasibilities.

\section{Introduction}

The current version of SDPT3, version 4.0, is designed to solve conic programming problems whose constraint cone is a product of semidefinite cones, second-order cones, nonnegative orthants and Euclidean spaces, and whose objective function is the sum of linear functions and log-barrier terms associated with the constraint cones. It solves the following standard form of such problems, henceforth called standard SQLP problems:

$$
\begin{aligned}
& \text { (P) } \min \sum_{j=1}^{n_{s}}\left[\left\langle c_{j}^{s}, x_{j}^{s}\right\rangle-\nu_{j}^{s} \log \operatorname{det}\left(x_{j}^{s}\right)\right]+\sum_{i=1}^{n_{q}}\left[\left\langle c_{i}^{q}, x_{i}^{q}\right\rangle-\nu_{i}^{q} \log \gamma\left(x_{i}^{q}\right)\right] \\
& +\left\langle c^{l}, x^{l}\right\rangle-\sum_{k=1}^{n_{l}} \nu_{k}^{l} \log x_{k}^{l} \quad+\left\langle c^{u}, x^{u}\right\rangle \\
& \text { s.t. } \sum_{j=1}^{n_{s}} \mathcal{A}_{j}^{s}\left(x_{j}^{s}\right) \quad+\sum_{i=1}^{n_{q}} A_{i}^{q} x_{i}^{q} \quad+A^{l} x^{l} \quad+A^{u} x^{u}=b, \\
& x_{j}^{s} \in K_{s}^{s_{j}} \forall j, \quad x_{i}^{q} \in K_{q}^{q_{i}} \forall i, \quad x^{l} \in K_{l}^{n_{l}}, \quad x^{u} \in \mathbb{R}^{n_{u}} .
\end{aligned}
$$

Here, $c_{j}^{s}, x_{j}^{s}$ lie in the space $\mathcal{S}^{s_{j}}$ of real symmetric matrices of order $s_{j}$ and $K_{s}^{s_{j}}$ is the cone of positive semidefinite symmetric matrices of the same order. Similarly, $c_{i}^{q}, x_{i}^{q}$ are vectors in $\mathbb{R}^{q_{i}}$ and $K_{q}^{q_{i}}$ is the quadratic or second-order cone defined by $K_{q}^{q_{i}}:=\left\{x=\left[x_{0} ; \bar{x}\right] \in \mathbb{R}^{q_{i}}: x_{0} \geq \sqrt{\bar{x}^{T} \bar{x}}\right\}$. Finally, $c^{l}, x^{l}$ are real vectors of dimension $n_{l}, K_{l}^{n_{l}}$ is the nonnegative orthant $\mathbb{R}_{+}^{n_{l}}$, and $c^{u}, x^{u}$ are real vectors of dimension $n_{u}$. In the notation above, $\mathcal{A}_{j}^{s}$ is the linear map from $K_{s}^{s_{j}}$ to $\mathbb{R}^{m}$ defined by

$$
\mathcal{A}_{j}^{s}\left(x_{j}^{s}\right)=\left[\left\langle a_{j, 1}^{s}, x_{j}^{s}\right\rangle ; \ldots ;\left\langle a_{j, m}^{s}, x_{j}^{s}\right\rangle\right],
$$

where $a_{j, 1}^{s}, \ldots, a_{j, m}^{s} \in \mathcal{S}^{s_{j}}$ are constraint matrices associated with the $j$ th semidefinite block variable $x_{j}^{s}$. The matrix $A_{i}^{q}$ is an $m \times q_{i}$ dimensional constraint matrix corresponding to the $i$ th quadratic block variable $x_{i}^{q}$, and $A^{l}$ and $A^{u}$ are the $m \times n_{l}$ and $m \times n_{u}$ dimensional constraint matrices corresponding to the linear block variable $x^{l}$ and the unrestricted block variable $x^{u}$, respectively. The notation $\langle p, q\rangle$ denotes the standard inner product in the appropriate space. For a given vector $u=\left[u_{0} ; \bar{u}\right]$ in a second-order cone, we define $\gamma(u):=\sqrt{u_{0}^{2}-\bar{u}^{T} \bar{u}}$. In the problem $(P), \nu_{j}^{s}, \nu_{i}^{q}$, and $\nu_{k}^{l}$ are given nonnegative parameters.

In this paper, the vector 2-norm and matrix Frobenius norm are denoted by $\|\cdot\|$. We use the MATLAB notation $[U ; V]$ to denote the matrix obtained by appending $V$ below the last row of $U$. For a given matrix $U$, we use the notation $U(k,:)$ and $U(:, k)$ to denote the $k$ th row and column of $U$, respectively. 
Let svec : $\mathcal{S}^{n} \rightarrow \mathbb{R}^{n(n+1) / 2}$ be the vectorization operator on symmetric matrices defined by $\operatorname{svec}(X)=\left[X_{11}, \sqrt{2} X_{12}, X_{22}, \ldots, \sqrt{2} X_{1 n}, \ldots, \sqrt{2} X_{n-1, n}, X_{n n}\right]^{T}$. For computational purposes, it is convenient to identify $\mathcal{A}_{j}^{s}$ with the following $m \times \bar{s}_{j}$ matrix (where $\left.\bar{s}_{j}=s_{j}\left(s_{j}+1\right) / 2\right)$ :

$$
A_{j}^{s}=\left[\operatorname{svec}\left(a_{j, 1}^{s}\right) ; \ldots ; \operatorname{svec}\left(a_{j, m}^{s}\right)\right] .
$$

With the matrix representation of $\mathcal{A}_{j}^{s}$, we have that $\mathcal{A}_{j}^{s}\left(x_{j}^{s}\right)=A_{j}^{s} \operatorname{svec}\left(x_{j}^{s}\right)$.

The software also solves the dual problem associated with the problem $(P)$ above:

(D) $\max b^{T} y+\sum_{j=1}^{n_{s}}\left[\nu_{j}^{s} \log \operatorname{det}\left(z_{j}^{s}\right)+s_{j} \nu_{j}^{s}\left(1-\log \nu_{j}^{s}\right)\right]$

$$
\begin{aligned}
& +\sum_{i=1}^{n_{q}}\left[\nu_{i}^{q} \log \gamma\left(z_{i}^{q}\right)+\nu_{i}^{q}\left(1-\log \nu_{i}^{q}\right)\right]+\sum_{k=1}^{n_{l}}\left[\nu_{k}^{l} \log z_{k}^{l}+\nu_{k}^{l}\left(1-\log \nu_{k}^{l}\right)\right] \\
& \text { s.t. } \quad\left(\mathcal{A}_{j}^{s}\right)^{T} y+z_{j}^{s}=c_{j}^{s}, \quad z_{j}^{s} \in K_{s}^{s_{j}}, \quad j=1 \ldots, n_{s} \\
& \quad\left(A_{i}^{q}\right)^{T} y+z_{i}^{q}=c_{i}^{q}, \quad z_{i}^{q} \in K_{q}^{q_{i}}, \quad i=1 \ldots, n_{q} \\
& \left(A^{l}\right)^{T} y+z^{l}=c^{l}, \quad z^{l} \in K_{l}^{n_{l}}, \\
& \left(A^{u}\right)^{T} y \quad=c^{l}, \quad y \in \mathbb{R}^{m} .
\end{aligned}
$$

In the notation above, $\left(\mathcal{A}_{j}^{s}\right)^{T}$ is the adjoint of $\mathcal{A}_{j}^{s}$ defined by $\left(\mathcal{A}_{j}^{s}\right)^{T} y=\sum_{k=1}^{m} y_{k} a_{j, k}^{s}$.

For later convenience, we introduce the following notation:

$$
x^{s}=\left(x_{1}^{s} ; \ldots ; x_{n_{s}}^{s}\right), \quad x^{q}=\left[x_{1}^{q} ; \ldots ; x_{n_{q}}^{q}\right], \quad A^{q}=\left[A_{1}^{q}, \ldots, A_{n_{q}}^{q}\right],
$$

where the notation $\left(x_{1}^{s} ; \ldots ; x_{n_{s}}^{s}\right)$ means that the objects $x_{j}^{s}$ are placed in a column format. We define $c^{s}, z^{s}, c^{q}$, and $z^{q}$ analogously. Let $\mathcal{A}^{s}\left(x^{s}\right)=\sum_{j=1}^{n_{s}} \mathcal{A}_{j}^{s}\left(x_{j}^{s}\right),\left(\mathcal{A}^{s}\right)^{T} y=$ $\left(\left(\mathcal{A}_{1}^{s}\right)^{T} y ; \ldots ;\left(\mathcal{A}_{n_{s}}^{s}\right)^{T} y\right)$, and $c=\left(c^{s} ; c^{q} ; c^{l} ; c^{u}\right), x=\left(x^{s} ; x^{q} ; x^{l} ; x^{u}\right), z=\left(z^{s} ; z^{q} ; z^{l} ; 0\right)$. Finally, we define

$$
\begin{gathered}
\mathcal{A}(x)=\mathcal{A}^{s}\left(x^{s}\right)+A^{q} x^{q}+A^{l} x^{l}+A^{u} x^{u}, \quad \mathcal{A}^{T}(y)=\left(\left(\mathcal{A}^{s}\right)^{T} y ;\left(A^{q}\right)^{T} y ;\left(A^{l}\right)^{T} y ;\left(A^{u}\right)^{T} y\right), \\
K=K_{s}^{s_{1}} \times \cdots \times K_{s}^{s_{n_{s}}} \times K_{q}^{q_{1}} \times \cdots \times K_{q}^{q_{n_{q}}} \times K_{l}^{n_{l}} \times \mathbb{R}^{n_{u}}, \\
K^{*}=K_{s}^{s_{1}} \times \cdots \times K_{s}^{s_{n_{s}}} \times K_{q}^{q_{1}} \times \cdots \times K_{q}^{q_{n_{q}}} \times K_{l}^{n_{l}} \times\{0\},
\end{gathered}
$$

so that the equality constraints of $(P)$ and $(D)$ can be written more compactly as follows:

$$
\mathcal{A}(x)=b, \quad x \in K, \quad \mathcal{A}^{T}(y)+z=c, \quad z \in K^{*} .
$$

The matrix representation of $\mathcal{A}$ is given by

$$
A=\left[A_{1}^{s}, \ldots, A_{n_{s}}^{s}, A^{q}, A^{l}, A^{u}\right] .
$$


The software package was originally developed to provide researchers in semidefinite programming with a collection of reasonably efficient and robust algorithms that could solve general SDPs (semidefinite programming problems) with matrices of dimensions of the order of a hundred. The current release expands the family of problems solvable by the software and made several enhancements described below.

1. This version is faster than the previous releases, e.g. [34], [39], especially on large sparse problems, and consequently can solve larger problems.

2. The current release can also solve problems that have explicit log-barrier terms in the objective functions. Hence determinant maximization problems can be solved.

3. The solver is more robust in handling unrestricted variables.

4. Low-rank structures in the constraint matrices associated with the semidefinite blocks can be exploited to improve computational efficiency and memory requirements.

5. The current release also implements primal-dual predictor-corrector path-following algorithms for solving a 3-parameter homogeneous self-dual model of $(P)$ and $(D)$.

6. Routines are provided to compute the geometric measures proposed by Freund [9] for $(P)$ and $(D)$. These geometric measures give information on the "thickness" of the feasible regions of $(P)$ and $(D)$.

All the numerical experiments in this paper were performed on an Intel Xeon 3.0 GHz personal computer with 4GB of physical memory using MATLAB version 7.6 on a Linux operating system.

Organization of the paper. In Section 2, we describe the representation of SQLP data by cell arrays. The SQLP solver sqlp.m in our software is described in Section 3. In Section 4, we present a few SQLP examples to illustrate the usage of our software. Implementation details such as the computation of search directions are given in Section 5. In Section 6, we describe a 3-parameter homogeneous self-dual model for the problem $(P)$ without logarithmic terms and unrestricted variables. In Section 7, the geometric measures of Freund and their computation are presented. Finally, the last section reports computational results obtained by SDPT3 on about 430 SQLP problems.

Installation. The current version is written in MATLAB version 7.4 or later releases. It is available from the internet site:

http://www.math.nus.edu.sg/ mattohkc/sdpt3.html

Our software uses a number of Mex routines generated from $\mathrm{C}$ programs written to carry out certain operations that MATLAB is not efficient at. In particular, operations such as extracting selected elements of a matrix, and performing arithmetic operations on these selected elements are all done in C. As an example, the vectorization 
operation svec is coded in the C program mexsvec.c. To install SDPT3 and generate these Mex routines, the user can simply follow the steps below:

(a) unzip SDPT3-4.0.zip;

(b) run MATLAB in the directory SDPT3-4.0;

(c) run the m-file Installmex.m.

After that, to see whether you have installed SDPT3 correctly, run the m-files:

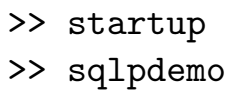

\section{Cell array representation of problem data}

Our implementation exploits the block structure of the given SQLP problem. In the internal representation of the problem data, we classify each semidefinite block into one of the following two types:

1. a dense or sparse matrix of order greater than or equal to 100;

2. a sparse block-diagonal matrix consisting of numerous sub-blocks each of order less than 100 .

The reason for using the sparse matrix representation to handle the case when we have numerous small diagonal blocks is that it is less efficient for MATLAB to work with a large number of cell array elements compared to working with a single cell array element consisting of a large sparse block-diagonal matrix. Technically, no problem will arise if one chooses to store the small blocks individually instead of grouping them together as a sparse block-diagonal matrix.

For the quadratic part, we typically group all quadratic blocks (small or large) into a single block, though it is not mandatory to do so. If there are a large number of small blocks, it is advisable to group them all together as a single large block consisting of numerous small sub-blocks for the same reason we just mentioned.

Let $L$ be the total number of blocks in the SQLP problem. If all the various types of blocks are present in $(P)$, then $L=n_{s}+n_{q}+2$. For each SQLP problem, the block structure of the problem data is described by an $L \times 2$ cell array named blk. The content of each of the elements of the cell arrays is given as follows. If the $j$ th block is a semidefinite block consisting of a single block of size $s_{j}$, then

$$
\begin{aligned}
& \operatorname{blk}\{j, 1\}=' s^{\prime}, \quad \operatorname{blk}\{j, 2\}=\left[s_{j}\right], \\
& \operatorname{At}\{j\}=\left[\overline{\mathbf{s}}_{j} \times m \text { sparse }\right], \\
& C\{j\}, X\{j\}, Z\{j\}=\left[\mathbf{s}_{j} \times s_{j} \text { double or sparse }\right],
\end{aligned}
$$

where $\overline{\mathbf{s}}_{j}=\mathbf{s}_{j}\left(\mathbf{s}_{j}+1\right) / 2$.

If the $j$ th block is a semidefinite block consisting of numerous small sub-blocks, say $p$ of them, of orders $\mathbf{s}_{j 1}, \mathbf{s}_{j 2}, \ldots, \mathbf{s}_{j p}$ such that $\sum_{k=1}^{p} \mathbf{s}_{j \mathrm{k}}=\mathbf{s}_{\mathbf{j}}$, then 


$$
\begin{aligned}
& \operatorname{blk}\{j, 1\}=' s^{\prime}, \quad \operatorname{blk}\{j, 2\}=\left[\begin{array}{lll}
s_{j 1}, & s_{j 2}, \cdots, & s_{j p}
\end{array}\right], \\
& \operatorname{At}\{j\}=\left[\bar{s}_{j} \times m \text { sparse }\right], \\
& C\{j\}, X\{j\}, Z\{j\}=\left[s_{j} \times s_{j} \text { sparse }\right],
\end{aligned}
$$

where $\bar{s}_{j}=\sum_{k=1}^{p} s_{j k}\left(s_{j k}+1\right) / 2$.

Notice that we store all the constraint matrices associated with the $j$ th semidefinite block in vectorized form as a single $\bar{s}_{j} \times m$ matrix where the $k$ th column of this matrix corresponds to the $k$ th constraint matrix. That is, $\operatorname{At}\{\mathrm{j}\}(:, \mathrm{k})=$ $\operatorname{svec}\left(\operatorname{blk}(j,:), a_{j, k}^{s}\right)$. (Here svec has a new argument because, if the $j$ th semidefinite block consists of several small sub-blocks, it needs to concatenate the svec's of each sub-block.)

The above storage scheme for the data matrix $A_{j}^{s}$ associated with the semidefinite blocks of the SQLP problem represents a departure from earlier versions (version 2.3 or earlier) of our implementation, such as the one described in [34]. Previously, the constraint matrices were stored in an $n_{s} \times m$ cell array AA, where $\operatorname{AA}\{\mathrm{j}, \mathrm{k}\}=a_{j, k}^{s}$, and it was stored as an individual matrix in either dense or sparse format. The data format we used in earlier versions of SDPT3 was more natural, but our current data representation was adopted for the sake of computational efficiency. The reason for such a change is again due to the fact that it is less efficient for MATLAB to work with a cell array with many cells. But note that it is easy to use the function svec.m provided in SDPT3 to convert AA into the new storage scheme as follows: $A t(j)=$ $\operatorname{svec}(\operatorname{blk}(j,:), A A(j,:))$.

While we now store the constraint matrix in vectorized form, the parts of the iterates $\mathrm{X}$ and $\mathrm{Z}$ corresponding to semidefinite blocks are still stored as matrices, since that is how the user wants to access them.

The data storage scheme corresponding to quadratic, linear, and unrestricted blocks is rather straightforward. If the $i$ th block is a quadratic block consisting of numerous sub-blocks, say $p$ of them, of dimensions $\mathrm{q}_{\mathrm{i} 1}, \mathrm{q}_{\mathrm{i} 2}, \ldots, \mathrm{q}_{\mathrm{ip}}$ such that $\sum_{k=1}^{p} \mathrm{q}_{\mathrm{ik}}=\mathrm{q}_{\mathrm{i}}$, then

$$
\begin{aligned}
& \operatorname{blk}\{i, 1\}=' q^{\prime}, \quad \operatorname{blk}\{i, 2\}=\left[q_{i 1}, q_{i 2}, \cdots, q_{i p}\right], \\
& \operatorname{At}\{i\}=\left[q_{i} \times m \text { sparse }\right], \\
& C\{i\}, X\{i\}, Z\{i\}=\left[q_{i} \times 1 \text { double or sparse }\right] .
\end{aligned}
$$

If the $k$ th block is the linear block, then

$$
\begin{aligned}
& \operatorname{blk}\{\mathrm{k}, 1\}={ }^{\prime} \mathrm{l}, \quad, \quad \mathrm{blk}\{\mathrm{k}, 2\}=\mathrm{n}_{1}, \\
& \text { At }\{\mathrm{k}\}=\left[\mathrm{n}_{1} \times \mathrm{m} \text { sparse }\right], \\
& \mathrm{C}\{\mathrm{k}\}, \mathrm{X}\{\mathrm{k}\}, \mathrm{Z}\{\mathrm{k}\}=\left[\mathrm{n}_{1} \times 1 \text { double or sparse }\right] .
\end{aligned}
$$

Similarly, if the $k$ th block is the unrestricted block, then

$$
\begin{aligned}
& \mathrm{blk}\{\mathrm{k}, 1\}=\text { 'u', } \mathrm{blk}\{\mathrm{k}, 2\}=\mathrm{n}_{\mathrm{u}}, \\
& \operatorname{At}\{\mathrm{k}\}=\left[\mathrm{n}_{\mathrm{u}} \mathrm{xm} \text { sparse }\right], \\
& \mathrm{C}\{\mathrm{k}\}, \mathrm{X}\{\mathrm{k}\}, \mathrm{Z}\{\mathrm{k}\}=\left[\mathrm{n}_{\mathrm{u}} \times 1 \text { double or sparse }\right] .
\end{aligned}
$$


(It is possible to have several linear or unrestricted blocks, but it is more efficient to reformulate such a problem by combining all linear blocks and similarly all unrestricted blocks.)

\subsection{Specifying the block structure of problems}

Our software requires the user to specify the block structure of the SQLP problem. Although no technical difficulty will arise if the user chooses to lump a few blocks together and consider it as a single large block, the computational time can be dramatically different. For example, the problem qpG11 in the SDPLIB library [2] actually has the block structure: $\operatorname{blk}\{1,1\}={ }^{\prime} \mathrm{s}^{\prime}, \operatorname{blk}\{1,2\}=800, \operatorname{blk}\{2,1\}=$ ' $l$ ', $\operatorname{blk}\{2,2\}=800$, but the structure specified in the library is $\operatorname{blk}\{1,1\}=$ ' $\mathrm{s}$ ', $\mathrm{blk}\{1,2\}=1600$. That is, in the former, the linear variables are explicitly identified, rather than being part of a large sparse semidefinite block. The difference in the running time for specifying the block structure differently is dramatic: the former representation is at least six times faster when the HKM direction is used, besides using much less memory space.

It is thus crucial to present problems to the algorithms correctly. We could add our own preprocessor to detect this structure, but believe users are aware of linear variables present in their problems. Unfortunately the versions of qpG11 (and also qpG51) in SDPLIB do not show this structure explicitly. In our software, we provide an m-file, detect_lblk.m, to detect problems with linear variables. The user can call this m-file after loading the problem data into MATLAB as follows:

$\gg[\mathrm{blk}, \mathrm{At}, \mathrm{C}, \mathrm{b}]=\operatorname{read}_{\mathrm{s}} \mathrm{sdpa}\left({ }^{\prime} \cdot / \mathrm{sdplib} / \mathrm{qpG} 11\right.$. dat-s' $\left.{ }^{\prime}\right)$;

> $[\mathrm{blk} 2, \mathrm{At2}, \mathrm{C} 2]=\operatorname{detect}_{-} \mathrm{lblk}(\mathrm{blk}, \mathrm{At}, \mathrm{C}, \mathrm{b})$;

Internally, the solvers in SDPT3 would automatically call detect_lblk.m to detect the presence of linear variables in a semidefinite block before solving $(P)$ and $(D)$.

\subsection{Storing constraint matrices with low-rank structures}

A new feature of the current version of SDPT3 is that it can exploit low-rank structures present in the constraint matrices associated with the semidefinite blocks. To do so, the user needs to specify the low-rank structures in the constraint matrices explicitly when coding the problem data. The purpose here is to explain how this is done.

Suppose the $j$ th row of blk corresponds to a semidefinite block. To simplify implementation, we exploit possible low-rank structures only when this semidefinite block is a single block. That is, $\operatorname{blk}\{\mathbf{j}, 2\}=\left[\mathbf{s}_{\mathbf{j}}\right]$. Suppose that the first $p$ matrices, $a_{j, 1}^{s}, \ldots a_{j, p}^{s}$, have no low-rank structures, and the remaining matrices $a_{j, p+1}^{s}, \ldots, a_{j, m}^{s}$ have such structures with

$$
a_{j, k}^{s}=V_{k} D_{k} V_{k}^{T}, \quad k=p+1, \ldots, m,
$$

where $V_{k} \in \mathbb{R}^{s_{j} \times r_{j, k}}$ is a low-rank matrix with $r_{j, k} \ll s_{j}$, and $D_{k} \in \mathbb{R}^{r_{j, k} \times r_{j, k}}$ is a symmetric matrix. The low-rank structures of these matrices should be recorded as follows: 


$$
\begin{aligned}
& \operatorname{blk}\{j, 1\}=' s^{\prime}, \quad \operatorname{blk}\{j, 2\}=\left[s_{j}\right], \quad \operatorname{blk}\{j, 3\}=\left[r_{j, p+1}, \ldots, r_{j, m}\right], \\
& \operatorname{At}\{j, 1\}=\left[\bar{s}_{j} x p \text { sparse }\right], \quad \operatorname{At}\{j, 2\}=\left[\mathrm{V}_{j, p+1}, \ldots, V_{j, m}\right], \quad \operatorname{At}\{j, 3\}=d d,
\end{aligned}
$$

where dd is a 4-column matrix that records the non-zero elements of $D_{k}, k=p+$ $1, \ldots, m$, and a row (say, $i$ th row) of dd has the following form:

$$
\mathrm{d}(\mathrm{i},:)=[\text { constraint number }-p \text {, row index, column index, non-zero value }] .
$$

If all the matrices $D_{k}$ are diagonal, then the user can simply set dd to be the following column vector:

$$
\mathrm{dd}=\left[\operatorname{diag}\left(D_{p+1}\right) ; \ldots ; \operatorname{diag}\left(D_{m}\right)\right] .
$$

In the subdirectory Examples, we give an m-file randlowranksdp.m to generate random SDP problems with low-rank constraint matrices, whose calling syntax is:

$[\mathrm{blk}, \mathrm{At}, \mathrm{C}, \mathrm{b}, \mathrm{bblk}, \mathrm{AAt}]=\operatorname{randlowranksdp}(\mathrm{n}, \mathrm{p}, \mathrm{m} 2, \mathrm{r})$

It will generate an SDP where the first $\mathrm{p}$ constraint matrices have no low-rank structures, and the remaining $\mathrm{m} 2$ matrices have low-rank structures and each matrix has rank $r$. The output [blk,At, C,b] explicitly describes the low-rank structure as above, while [bblk, AAt , C, b] encodes the same SDP, but without including the lowrank structure information.

\section{The main functions: sqlp.m, HSDsqlp.m, sdpt3.m}

The main algorithm implemented in SDPT3 for solving $(P)$ and $(D)$ is an infeasible primal-dual path-following algorithm, described in detail in Appendix A. At each iteration, we first compute a predictor search direction aimed at decreasing the duality gap as much as possible. After that, the algorithm generates a Mehrotra-type corrector step [23] with the intention of keeping the iterates close to the central path. However, we do not impose any neighborhood restrictions on our iterates. ${ }^{1}$ Initial iterates need not be feasible - the algorithm tries to achieve feasibility and optimality of its iterates simultaneously. It should be noted that in our implementation, the user has the option to use a primal-dual path-following algorithm that does not use corrector steps.

The main routine that corresponds to Algorithm IPC described in Appendix A for solving $(P)$ and $(D)$ is sqlp.m, whose calling syntax is as follows:

$$
[\text { obj }, \mathrm{X}, \mathrm{y}, \mathrm{Z} \text {, info, runhist }]=\operatorname{sqlp}(\mathrm{blk}, \mathrm{At}, \mathrm{C}, \mathrm{b}, \text { OPTIONS }, \mathrm{X} 0, \mathrm{y} 0, \mathrm{Z0}) \text {. }
$$

For an SQLP problem without logarithmic terms or unrestricted variables $x^{u}$, we also implemented an algorithm that is analogous to Algorithm IPC for a 3-parameter homogeneous self-dual model of $(P)$. The routine for solving the HSD model of $(P)$ is HSDsqlp.m, with the following syntax:

\footnotetext{
${ }^{1}$ This strategy works well on most of the problems we tested. However, it should be noted that the occasional failure of the software on problems with poorly chosen initial iterates is likely due to the lack of a neighborhood enforcement in the algorithm.
} 


$$
\text { [obj, X, y, Z, info, runhist }]=\text { HSDsqlp (blk, At, C , b , OPTIONS, XO, y0, Z0). }
$$

Note that if there are unrestricted variables $x^{u}$ present in $(P)$, HSDsqlp.m can still be called to solve the problem since it would automatically express $x^{u}$ as $x^{u}=x_{+}^{u}-x_{-}^{u}$ with $x_{+}^{u}, x_{-}^{u} \geq 0$ to reformulate $(P)$ into an SQLP without unrestricted variables. Our numerical experience has indicated that for an SQLP with unrestricted variables in $(P)$, HSDsqlp.m would typically deliver more accurate solutions than sqlp.m since the former generally would encounter less severe numerical difficulties compared to the latter. But for an SQLP problem without unrestricted variables, HSDsqlp.m generally would take more iterations than sqlp.m to solve the problem to the same accuracy. Based on the consideration of computational efficiency and attainable accuracy/robustness, we have a hybrid solver sdpt3.m that would automatically choose between sqlp.m and HSDsqlp.m based on the characteristics of the SQLP problem to be solved. Again, the calling syntax of sdpt3.m is similar to that for sqlp.m.

\section{Input arguments.}

blk: a cell array describing the block structure of the SQLP problem.

At, C, b: SQLP data.

OPTIONS: a structure array of parameters (optional).

xo, y0, zo: an initial iterate (optional).

If the input argument OPTIONS is omitted, default values specified in the function sqlparameters.m are used. More detail on OPTIONS is given in Section 3.1.

\section{Output arguments.}

The names chosen for the output arguments explain their contents. The argument info is a structure array containing performance information such as info.termcode, info.obj, info.gap, info.pinfeas, info.dinfeas, info.cputime whose meanings are explained in sqlp.m. The argument runhist is a structure array which records the history of various performance measures during the run; for example, runhist.gap records the complementarity gap at each interior-point iteration.

Note that, while $(\mathrm{X}, \mathrm{y}, \mathrm{Z})$ normally gives approximately optimal solutions, if info.termcode is 1 the problem is suspected to be primal infeasible and $(y, z)$ is an approximate certificate of infeasibility, with $\mathrm{b}^{T} \mathrm{y}=1, \mathrm{z}$ in the appropriate cone, and $\mathrm{A}^{T} \mathrm{y}+\mathrm{Z}$ small, while if info.termcode is 2 the problem is suspected to be dual infeasible and $\mathrm{X}$ is an approximate certificate of infeasibility, with $\langle\mathrm{C}, \mathrm{X}\rangle=-1, \mathrm{X}$ in the appropriate cone, and AX small. Note that $\mathrm{A}$ is defined in (2).

\section{Caveats.}

(a) The user should be aware that SQLP is more complicated than linear programming. For example, it is possible that both primal and dual problems are feasible, but their optimal values are not equal. Also, either problem may be infeasible without there being a certificate of that fact (so-called weak infeasibility). In such cases, our software package is likely to terminate after some iterations with an indication of short step-length or lack of progress. Also, even if there is a certificate of infeasibility, 
our infeasible-interior-point methods may not find it. In our very limited testing on strongly infeasible problems, our algorithms have been quite successful in detecting infeasibility.

(b) Since our algorithm is a primal-dual method storing the primal iterate X, it cannot exploit common sparsity in $\mathrm{C}$ and the constraint matrices as well as dual methods or nonlinear-programming based methods. Thus our software may not be able to handle dense or sparse semidefinite blocks (with a single block) with order more than 3000 on an average PC available in 2010.

(c) Our interior-point algorithms are designed based on the existence of a central path in the interior of the primal-dual feasible region of $(P)$ and $(D)$. For problems where the primal-dual feasible region is non-empty but has an empty interior, our SQLP solver can generally still deliver a reasonably good approximate optimal solution, but the solver tends to encounter numerical difficulties before a high accuracy solution can be obtained.

\subsection{The structure array OPTIONS for parameters}

sqlp.m uses a number of parameters which are specified in a MATLAB structure array called OPTIONS in the m-file sqlparameters.m. If desired, the user can change the values of these parameters. The meaning of the specified fields in OPTIONS are given in the m-file itself. As an example, if the user does not wish to use corrector steps in Algorithm IPC, then he/she can do so by setting OPTIONS . predcorr $=0$. If the user wants to use a fixed value, say 0.98, for the step-length parameter $\bar{\gamma}$ in Algorithm IPC instead of the adaptive strategy used in the default, he/she can achieve that by setting OPTIONS.gam $=0.98$. Similarly, if the user wants to solve the SQLP problem to an accuracy tolerance of $1 \mathrm{e}-4$ instead of the default value of $1 \mathrm{e}-8$ while using the default values for all other parameters, he/she only needs to set OPTIONS. gaptol = $1 \mathrm{e}-4$.

The defaults in sqlparameters.m assume that the parameters $\nu_{j}^{s}, \nu_{i}^{q}, \nu_{k}^{l}$ in $(P)$ are all 0. For an SQLP problem where some of the parameters $\nu_{j}^{s}, \nu_{i}^{q}, \nu_{k}^{l}$ are positive, the user needs to specify an $L \times 1$ cell array OPTIONS . parbarrier to store the values of these parameters (including zeros) as follows. If the $j$ th block is a semidefinite block consisting of one or more sub-blocks, say $p$ of them, of orders $\mathbf{s}_{j 1}, \mathbf{s}_{j 2}, \ldots, \mathbf{s}_{j p}$, then

$$
\text { OPTIONS.parbarrier }\{j\}=\left[\begin{array}{llll}
\nu_{j 1}^{\mathbf{s}}, & \nu_{j 2}^{\mathbf{s}}, & \cdots, & \nu_{j p}^{\mathbf{s}}
\end{array}\right] .
$$

If the $i$ th block is a quadratic block consisting of one or more sub-blocks, say $p$ of them, of dimensions $\mathrm{q}_{i 1}, \mathrm{q}_{\mathrm{i} 2}, \ldots, \mathrm{q}_{\mathrm{ip}}$, then

$$
\text { OPTIONS.parbarrier }\{i\}=\left[\begin{array}{llll}
\nu_{i 1}^{\mathrm{q}}, & \nu_{\mathrm{i} 2}^{\mathrm{q}}, & \cdots, & \nu_{\mathrm{ip}}^{\mathrm{q}}
\end{array}\right] \text {. }
$$

If the $k$ th block is the linear block, then

$$
\text { OPTIONS.parbarrier }\{\mathrm{k}\}=\left[\begin{array}{llll}
\nu_{1}^{1}, & \nu_{2}^{1}, \cdots, & \nu_{\mathrm{n}_{1}}^{1}
\end{array}\right],
$$

while if the $k$ th block is the unrestricted block, then 
We can solve a DIMACS test problem in a similar manner.

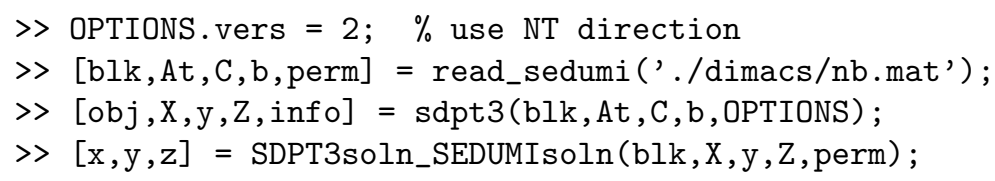

In the above, we call the hybrid solver sdpt3.m to solve the SQLP, and in the last line, we convert the solution in SDPT3 format to SeDuMi format.

\subsection{Stopping criteria}

We define

$$
\begin{aligned}
n & =\sum_{\left\{j: \nu_{j}^{s}=0\right\}} s_{j}+\sum_{\left\{i: \nu_{i}^{q}=0\right\}} q_{i}+\left|\left\{k: \nu_{k}^{l}=0\right\}\right| \\
\mu(x, z) & =\frac{1}{n} \sum_{\alpha \in\{s, q, l\}} \sum_{j=1}^{n_{\alpha}} \begin{cases}\left\langle x_{j}^{\alpha}, z_{j}^{\alpha}\right\rangle & \text { if } \nu_{j}^{\alpha}=0 \\
0 & \text { otherwise. }\end{cases} \\
\text { gap } & =\langle x, z\rangle-\sum_{\left\{j: \nu_{j}^{s}>0\right\}} \nu_{j}^{s}\left(s_{j}+\log \operatorname{det}\left(x_{j}^{s} z_{j}^{s} / \nu_{j}^{s}\right)\right) \\
& -\sum_{\left\{i: \nu_{i}^{q}>0\right\}} \nu_{i}^{q}\left(1+\log \left(\gamma\left(x_{i}^{q}\right) \gamma\left(z_{i}^{q}\right) / \nu_{i}^{q}\right)\right)-\sum_{\left\{k: \nu_{k}^{l}>0\right\}} \nu_{k}^{l}\left(1+\log \left(x_{k}^{l} z_{k}^{l} / \nu_{k}^{l}\right)\right) .
\end{aligned}
$$

Note that if $n=0$, we define $\mu(x, z)=0$.

The algorithm is stopped when any of the following cases occur.

1. solutions with the desired accuracy have been obtained, i.e.,

$$
\phi:=\max \{\text { relgap, pinfeas, dinfeas }\} \leq \text { OPTIONS.gaptol }
$$

where

$$
\text { relgap }=\frac{\text { gap }}{1+|\langle c, x\rangle|+\left|b^{T} y\right|}, \text { pinfeas }=\frac{\|\mathcal{A}(x)-b\|}{1+\|b\|} \text {, dinfeas }=\frac{\left\|\mathcal{A}^{T}(y)+z-c\right\|}{1+\|c\|} .
$$

2. primal infeasibility is suggested because

$$
b^{T} y /\left\|\mathcal{A}^{T} y+z\right\|>10^{8} ;
$$

3. dual infeasibility is suggested because

$$
-c^{T} x /\|\mathcal{A} x\|>10^{8} ;
$$


4. slow progress is detected, measured by a rather complicated set of tests including

$$
\text { relgap }<\max \{\text { pinfeas, dinfeas }\}
$$

5. numerical problems are encountered, such as the iterates not being positive definite or the Schur complement matrix not being positive definite; or

6. the step sizes fall below $10^{-6}$.

\subsection{Initial iterates}

Our algorithms can start with an infeasible starting point. However, the performance of these algorithms is quite sensitive to the choice of the initial iterate. As observed in [13], it is desirable to choose an initial iterate that at least has the same order of magnitude as an optimal solution of the SQLP. If a feasible starting point is not known, we recommend that the following initial iterate be used:

$$
\begin{gathered}
y^{0}=0, \\
\left(x_{j}^{s}\right)^{0}=\xi_{j}^{s} I_{s_{j}}, \quad\left(z_{j}^{s}\right)^{0}=\eta_{j}^{s} I_{s_{j}}, \quad j=1, \ldots, n_{s}, \\
\left(x_{i}^{q}\right)^{0}=\xi_{i}^{q} e_{i}^{q}, \quad\left(z_{i}^{q}\right)^{0}=\eta_{i}^{q} e_{i}^{q}, \quad i=1, \ldots, n_{q}, \\
\left(x^{l}\right)^{0}=\xi^{l} e^{l}, \quad\left(z^{l}\right)^{0}=\eta^{l} e^{l}, \quad\left(x^{u}\right)^{0}=0,
\end{gathered}
$$

where $I_{s_{j}}$ is the identity matrix of order $s_{j}, e_{i}^{q}$ is the first $q_{i}$-dimensional unit vector, $e^{l}$ is the $l$-vector of all ones, and

$$
\begin{aligned}
\xi_{j}^{s} & =\max \left\{10, \sqrt{s_{j}}, s_{j} \max _{1 \leq k \leq m} \frac{1+\left|b_{k}\right|}{1+\left\|a_{j, k}^{s}\right\|_{F}}\right\}, \\
\eta_{j}^{s} & =\max \left\{10, \sqrt{s_{j}}, \max \left\{\left\|c_{j}^{s}\right\|_{F},\left\|a_{j, 1}^{s}\right\|_{F}, \ldots,\left\|a_{j, m}^{s}\right\|_{F}\right\}\right\}, \\
\xi_{i}^{q} & =\max \left\{10, \sqrt{q_{i}}, \sqrt{q_{i}} \max _{1 \leq k \leq m} \frac{1+\left|b_{k}\right|}{1+\left\|A_{i}^{q}(k,:)\right\|}\right\}, \\
\eta_{i}^{q} & =\max \left\{10, \sqrt{q_{i}}, \max \left\{\left\|c_{i}^{q}\right\|,\left\|A_{i}^{q}(1,:)\right\|, \ldots,\left\|A_{i}^{q}(m,:)\right\|\right\}\right\}, \\
\xi^{l} & =\max \left\{10, \sqrt{n_{l}}, \sqrt{n_{l}} \max _{1 \leq k \leq m} \frac{1+\left|b_{k}\right|}{1+\left\|A^{l}(k,:)\right\|}\right\}, \\
\eta^{l} & =\max \left\{10, \sqrt{n_{l}}, \max \left\{\left\|c^{l}\right\|,\left\|A^{l}(1,:)\right\|, \ldots,\left\|A^{l}(m,:)\right\|\right\}\right\} .
\end{aligned}
$$

By multiplying the identity matrix $I_{s_{j}}$ by the factors $\xi_{j}^{s}$ and $\eta_{j}^{s}$ for the semidefinite blocks, and similarly for the quadratic and linear blocks, the initial iterate has a better chance of having the appropriate order of magnitude.

The initial iterate above is set by calling infeaspt.m, with syntax 


$$
[\mathrm{XO}, \mathrm{y} 0, \mathrm{Z0}]=\text { infeaspt (blk, At, C, b, options, scalefac), }
$$

where options $=1$ (default) corresponds to the initial iterate just described, and options $=2$ corresponds to the choice where the blocks of $\mathrm{XO}, \mathrm{Z} 0$ are scalefac times identity matrices or unit vectors, and y0 is a zero vector.

\subsection{Preprocessing}

\section{Nearly dependent constraints.}

The primal-dual path-following algorithm we implemented assumes that the matrix $A$ in (2) has full column rank. But in our software, the presence of (nearly) dependent constraints is detected automatically, and warning messages are displayed if such constraints exist. When this happens, the user has the option of removing these (nearly) dependent constraints by calling a preprocessing routine to remove them by setting OPTIONS.rmdepconstr $=1$. The routine (checkdepconstr.m) we have coded to detect nearly dependent constraints is based on computing the sparse $L D L^{T}$ factorization of $A A^{T}$. Such a method is fast but is not as reliable as the method that is based on sparse $L U$ factorization of $A$.

\section{Detecting diagonal blocks.}

We provide the m-file, detect_lblk.m, to look for diagonal blocks in semidefinite blocks in the data: see Subsection 2.1 for the use of this subroutine.

\section{Detecting unrestricted blocks.}

We have provided a routine, detect_ublk.m, to detect unrestricted variables that have been modelled as the difference of two nonnegative variables.

\section{Complex data.}

In earlier versions, 2.3 or earlier, SDPT3 could directly handle complex data in SDP, i.e., the case where the constraint matrices are hermitian matrices. However, as problems with complex data rarely occur in practice, and in an effort to simplify the code, we removed this flexibility from subsequent versions.

Users can still solve an SDP with complex data using SDPT3-4.0. This is done by calling the m-file convertcmpsdp.m to convert the SDP into one with real data. But unlike the earlier versions, here we convert the problem into one with real data by doubling the size of the constraint matrices. Let $B$ be an $n \times n$ hermitian matrix. The conversion is based on the following equivalence:

$$
B \text { is positive semidefinite } \Leftrightarrow\left[\begin{array}{cc}
B^{R} & -B^{I} \\
B^{I} & B^{R}
\end{array}\right] \text { is positive semidefinite, }
$$

where $B^{R}$ and $B^{I}$ denote the real and imaginary parts of $B$, respectively. Note that since $B$ is hermitian, $B^{R}$ is symmetric and $B^{I}$ is skew-symmetric. 
Now suppose $C, A_{1}, \ldots A_{m}$ are given $n \times n$ hermitian matrices. Then $C-\sum_{k=1}^{m} y_{k} A_{k} \succeq$ 0 if and only

$$
\left[\begin{array}{cc}
C^{R} & -C^{I} \\
C^{I} & C^{R}
\end{array}\right]-\sum_{k=1}^{m} y_{k}^{R}\left[\begin{array}{cc}
A_{k}^{R} & -A_{k}^{I} \\
A_{k}^{I} & A_{k}^{R}
\end{array}\right]-\sum_{k=1}^{m} y_{k}^{I}\left[\begin{array}{cc}
-A_{k}^{I} & -A_{k}^{R} \\
A_{k}^{R} & -A_{k}^{I}
\end{array}\right] \succeq 0 .
$$

Notice that the matrices $\left[-A_{k}^{I},-A_{k}^{R} ; A_{k}^{R},-A_{k}^{I}\right]$ are skew-symmetric. For a complex $\mathrm{SDP}$, the vector $b$ must necessarily be real, and the linear term in the objective function in $(D)$ is replaced by $\left\langle b, y^{R}\right\rangle$. Since the skew symmetric matrices in (7) do not affect the positiveness condition and $y^{I}$ does not appear in the objective function in $(D)$, we can take $y_{I}^{k}=0, k=1, \ldots, m$.

Note that the conversion of a complex SDP into a real SDP based on (7) would double the storage and if the data is dense, the cost of each interior-point iteration for solving the resulting real SDP is about twice as expensive as that for solving the complex SDP directly.

To convert an SDP with complex data into one with only real data, the m-file convertcmpsdp.m has the calling syntax:

$[\mathrm{bblk}, \mathrm{AAt}, \mathrm{CC}, \mathrm{bb}]=\operatorname{convertcmpsdp}(\mathrm{blk}, \mathrm{At}, \mathrm{C}, \mathrm{b})$;

where AAt corresponds to the $m$ real symmetric constraint matrices in the first summation in (7), CC corresponds to the real constant matrix in (7), and $\mathrm{bb}=b^{R}$.

Internally, SDPT3 would automatically detect the presence of complex data in an SDP and convert it into one with only real data before solving it. But note that the current version of SDPT3 does not allow complex data corresponding to the quadratic (second-order cone) block.

\section{Rotated cones.}

Let $K_{r}^{n}(n \geq 3)$ be the rotated cone defined by

$$
K_{r}^{n}=\left\{x^{r}=[u ; v ; w] \in \mathbb{R}^{n}:\|w\|^{2} \leq 2 u v, u, v \geq 0\right\} .
$$

Note the constant "2" above. Define the symmetric orthogonal matrix $T_{n} \in \mathbb{R}^{n \times n}$ as follows:

$$
T_{n}=\left[\begin{array}{ccc}
1 / \sqrt{2} & 1 / \sqrt{2} & \\
1 / \sqrt{2} & -1 / \sqrt{2} & \\
& & I_{n-2}
\end{array}\right]
$$

It is easy to show that $x^{r} \in K_{r}^{n}$ if and only if $x^{q}:=T_{n} x^{r} \in K_{q}^{n}$, i.e., $T_{n} K_{r}^{n}=K_{q}^{n}$. Thus we can always convert a rotated cone variable into one belonging to a second-order cone.

In SDPT3-4.0, the user can code a rotated cone block consisting of several subblocks, say $p$ of them of dimension $r_{i 1}, \ldots, r_{i p}$, as follows:

$$
\operatorname{blk}\{i, 1\}={ }^{\prime} r ' ; \operatorname{blk}\{i, 2\}=\left[r_{i 1}, \ldots, r_{i p}\right] ;
$$


Let $D$ be the block diagonal matrix defined by $D=\operatorname{diag}\left(T_{r_{i 1}}, \ldots, T_{r_{i p}}\right)$. Internally, SDPT3 would convert such a rotated cone block and its associated data into a secondorder cone block as follows:

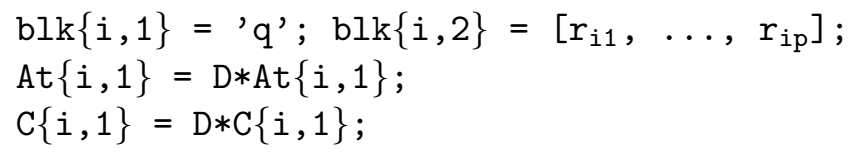

\section{Examples}

For an user to solve his SQLP problem using SDPT3, the first task he/she needs to perform is to write his problem in the standard form given in $(P)$ and then to code the problem data corresponding to the standard form. In the last few years, modelling languages such as CVX [8] and YALMIP [21] have been developed for advanced modelling and solution of convex optimization problems. These modelling languages would automatically convert an SQLP problem which is not necessarily expressed in the standard form given in $(P)$ or $(D)$ into the required standard form for solvers such as SDPT3 or SeDuMi. They would also generate the problem data corresponding to the standard form and call a solver to solve the problem. In CVX, SDPT3 is used as the default solver, but SeDuMi is also conveniently available. Similarly, YALMIP can also use SDPT3 or SeDuMi as its computational engine to solve SQLP problems.

For users who are interested only in solving small SQLP problems, we would advise them to use CVX or YALMIP to convert their problems into the required standard form and to generate the corresponding data as the process is usually quite tedious though mechanical. But for users who wish to solve large SQLP problems, it is sometimes highly beneficial for the users themselves to convert their problems into the required standard form rather than relying on CVX or YALMIP as these modelling languages may introduce large number of additional variables or significantly expand the problem dimensions when converting the problems into the standard form since they are based on automatic conversion procedures which do not take into account problem structures that users may be able to exploit.

The simplest way to learn how to convert an SQLP problem into the standard form given in $(P)$ or $(D)$ and to generate the corresponding problem data in SDPT3 format is through examples. (Note that the user can also store the problem data in either the SDPA or SeDuMi format, and then use the m-files read_sdpa.m or read_sedumi.m to read the data into SDPT3.) The subdirectory Examples in SDPT3 contains many such example files. Here we will just mention a few.

\subsection{Conversion of problems into the standard form}

As mentioned before, SQLP problems are usually not formulated in the standard form $(P)$ or $(D)$, and it is often quite tedious to convert such problems into the standard form. As such, users who are solving small to medium scale SQLPs are encouraged to use modelling languages such as CVX [8] or YALMIP [21] to automate the conversion process. To give the user an idea on how the conversion is done, here we shall just 
give an example to show how an SDP with linear equality and inequality constraints can be converted into the standard form given in $(P)$. Suppose we have an SDP of the following form:

$$
\begin{aligned}
\left(P_{1}\right) \min \quad\left\langle c^{s}, x^{s}\right\rangle & \\
\text { s.t. } \quad \mathcal{A}^{s}\left(x^{s}\right) & =b, \\
& \mathcal{B}^{s}\left(x^{s}\right) \leq d, \\
& x^{s} \in K_{s}^{n} .
\end{aligned}
$$

where $d \in \mathbb{R}^{p}$ and $\mathcal{B}^{s}$ is a linear map. By introducing a slack variable $x^{l}$, we can easily convert $\left(P_{1}\right)$ into standard form, namely,

$$
\begin{aligned}
\left(P_{1}^{*}\right) \min \quad\left\langle c^{s}, x^{s}\right\rangle+\left\langle c^{l}, x^{l}\right\rangle & \\
\text { s.t. } \quad & \mathcal{A}^{s}\left(x^{s}\right) \\
& \mathcal{B}^{s}\left(x^{s}\right)+B^{l} x^{l}=b, \\
& x^{s} \in K_{s}^{n} . \quad x^{l} \in K_{l}^{p},
\end{aligned}
$$

where $c^{l}=0$, and $B^{l}=I_{p \times p}$. With our use of cell arrays to store SQLP data, it is easy to take the problem data of $\left(P_{1}\right)$ and use them for the standard form $\left(P_{1}^{*}\right)$ as follows:

$$
\begin{array}{ll}
\operatorname{blk}\{1,1\}=' \mathrm{~s}^{\prime} ; & \operatorname{blk}\{1,2\}=\mathrm{n} ; \\
\operatorname{At}\{1,1\}=\left[A^{s} ; B^{s}\right]^{T} ; & \mathrm{C}\{1,1\}=c^{s} ; \\
\operatorname{blk}\{2,1\}='{ }^{\prime} ; & \operatorname{blk}\{2,2\}=\mathrm{p} ; \\
\operatorname{At}\{2,1\}=[\operatorname{sparse}(\mathrm{m}, \mathrm{p}) ; \operatorname{speye}(\mathrm{p}, \mathrm{p})]^{T} ; & \mathrm{C}\{2,1\}=c^{l} ; \\
\mathrm{b}=[\mathrm{b} ; \mathrm{d}] ; &
\end{array}
$$

In the subdirectory, an SDP of the form $\left(P_{1}\right)$ is coded in the example file max_kcut.m.

\subsection{The MAXCUT problem}

Let $\mathcal{S}_{+}^{n}$ be the space of $n \times n$ symmetric positive semidefinite matrices. Let $B$ be the weighted adjacency matrix of a graph. The SDP relaxation of the MAXCUT problem associated with $B$ has the following form:

$$
\begin{array}{ll}
\min & \langle C, X\rangle \\
\text { s.t. } & \operatorname{diag}(X)=e, \quad X \in \mathcal{S}_{+}^{n},
\end{array}
$$

where $e$ is the vector of ones, and $C=-(\operatorname{Diag}(B e)-B) / 4$. It is clear that we need the cell array, $b l k\{1,1\}={ }^{\prime} s^{\prime}, \operatorname{blk}\{1,2\}=n$, to record the block structure of the problem. The constraint matrices can be constructed conveniently via an $1 \times n$ cell array as follows: 


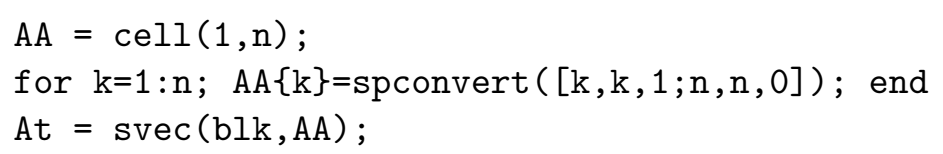

For more details, see the m-file maxcut.m in the subdirectory Examples. (We could also create a version of the problem explicitly showing the low-rank structure; however, as the constraint matrices are so sparse, this would not be more efficient.)

\subsection{D-optimal experiment design - an example with an explicit barrier term}

Given a set of points $\left\{v_{1}, \ldots, v_{p}\right\}$ in $\mathbb{R}^{n}$ with $n \leq p$, the D-optimal experiment design problem [40] needs to solve the following dual SQLP:

$$
\begin{aligned}
& \max \log \operatorname{det}(Z) \\
& \text { s.t. } \quad \sum_{k=1}^{p} y_{k}\left(-v_{k} v_{k}^{T}\right)+Z=0, \quad Z \in \mathcal{S}_{++}^{n} \\
& -y \quad+z^{l}=0, \quad z^{l} \in \mathbb{R}_{+}^{p} \\
& e^{T} y \quad=1, \quad y \in \mathbb{R}^{p} .
\end{aligned}
$$

The associated problem data can be coded in SDPT3 format as follows:

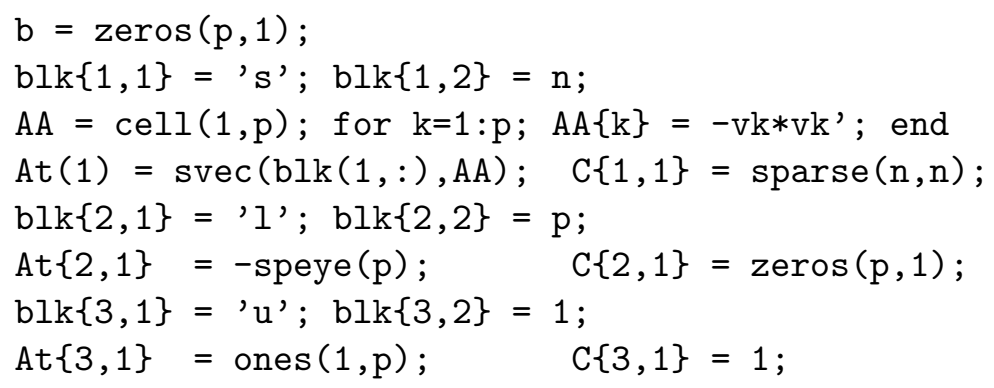

Because the problem contains an explicit log-barrier term in the objective function, we also need to set up OPTIONS. parbarrier as follows:

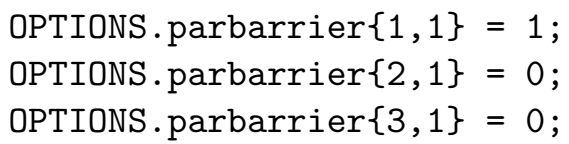

For more details, see the m-file Doptdesign.m in the subdirectory Examples.

The constraint matrices corresponding to the semidefinite block in this example are all rank-one matrices. The user can explicitly code such structures for SDPT3 as follows:

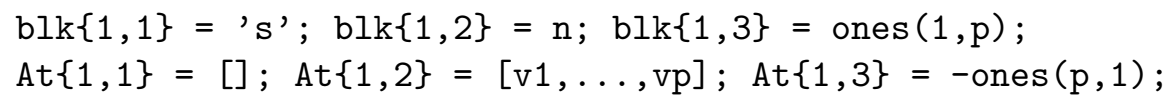


As an example on the potential speed up one may gain by exploiting the lowrank structure in the constraint matrices, we compare the times taken to solve two D-optimal experiment design problems with randomly generated data $\left\{v_{1}, \ldots, v_{p}\right\}$ in $\mathbb{R}^{n}$. For $n=200, p=400(n=100, p=400)$, the time taken to solve the problem without exploiting the low-rank structure is 157.1 (44.2) seconds, compared to just 2.9 (2.1) seconds when the low-rank structure is exploited.

\subsection{An LMI example}

Consider the following LMI problem [3]:

$$
\begin{aligned}
& \max -\eta \\
& \text { s.t. } \quad G Y+Y G^{T} \preceq 0 \\
& -Y \preceq \preceq-I \\
& Y-\eta I \quad \preceq 0 \\
& Y_{11} \quad=1, \quad Y \in \mathcal{S}^{n},
\end{aligned}
$$

where $G \in \mathbb{R}^{n \times n}$. This problem can be viewed as a dual SDP with $Y$ identified as a vector $y$ in $\mathbb{R}^{n(n+1) / 2}$. In this case, we have $\left(A_{1}^{s}\right)^{T} y=\operatorname{svec}\left(G \operatorname{smat}(y)+\operatorname{smat}(y) G^{T}\right)$, where smat is the inverse of svec. The SDP data can be generated for SDPT3 as follows:

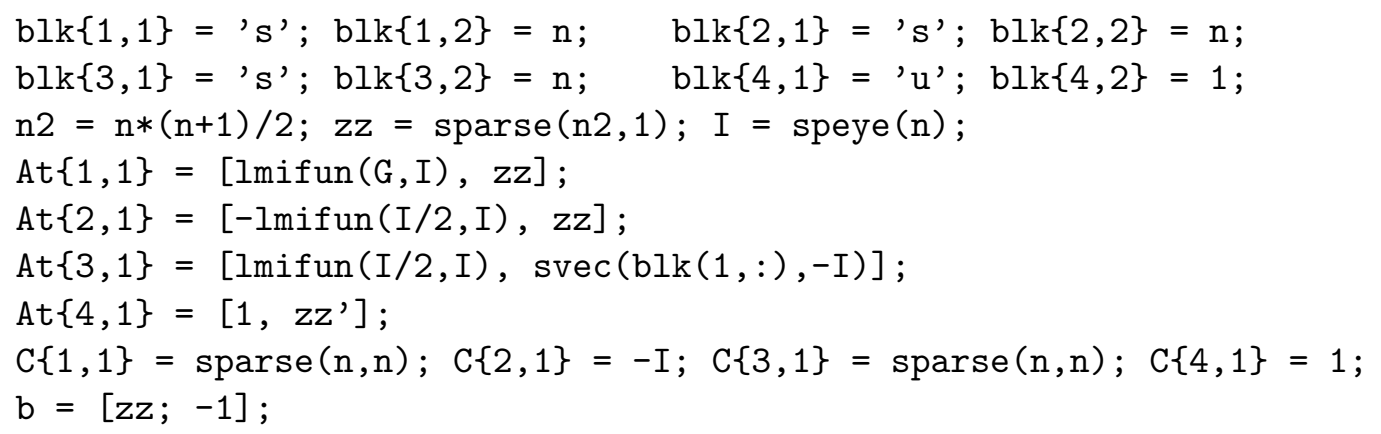

In the above, Imifun $(\mathrm{G}, \mathrm{H})$ is a function (available in Examples) that generates the matrix representation of the linear map $y \in \mathbb{R}^{n(n+1) / 2} \mapsto \operatorname{svec}\left(G \operatorname{smat}(y) H^{T}+\right.$ $\left.H \operatorname{smat}(y) G^{T}\right)$.

For more details, see the m-file lmiexamp1.m in the subdirectory Examples.

\subsection{The nearest correlation matrix problem}

Given an $n \times n$ symmetric matrix $H$, the nearest correlation matrix problem is the following [18]:

$$
\min _{X}\left\{\|H-X\|_{F}: \operatorname{diag}(X)=e, X \in \mathcal{S}_{+}^{n}\right\} .
$$

The above problem can be converted to the following SQLP:

$\min \left\{e_{1}^{T} y: \operatorname{diag}(X)=e, \operatorname{svec}(X)+\left[0, I_{n_{2}}\right] y=\operatorname{svec}(H), X \in \mathcal{S}_{+}^{n}, y \in Q^{n_{2}+1}\right\}$, 
where $n_{2}=n(n+1) / 2, I_{n_{2}}$ denotes the identity matrix of order $n_{2}$, and $Q^{n_{2}+1}$ denotes the second-order cone of dimension $n_{2}+1$. Also, $e_{1}$ denotes the first unit vector in $\left(n_{2}+1\right)$-space; in general, $e_{i}$ will denote the $i$ th unit vector of appropriate dimension. The corresponding SQLP data can be coded for SDPT3 as follows:

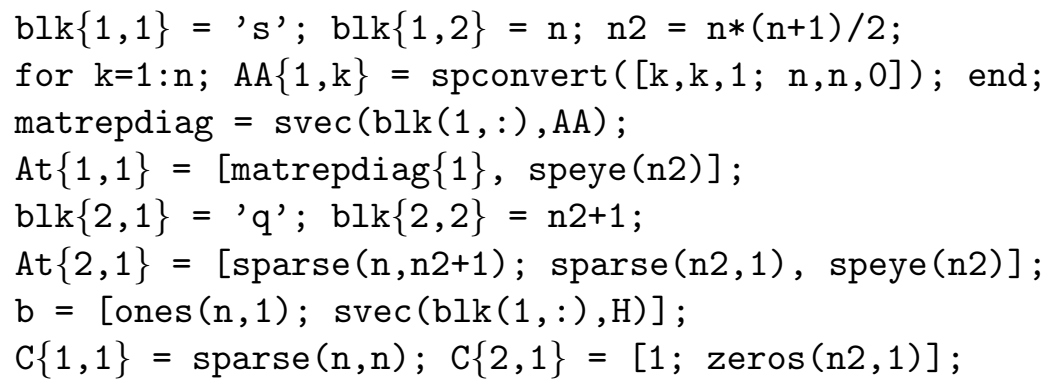

For more details, see the m-file corrmat. $m$ in the subdirectory Examples.

\subsection{A problem with a convex quadratic objective func- tion}

The previous example has a convex quadratic objective function in the primal problem. Here we consider the case where the dual problem has a concave quadratic objective function, namely,

$$
\max _{y, Z}\left\{b^{T} y-y^{T} H y: \mathcal{A}_{s}^{T} y+z_{s}=c_{s}, z_{s} \in \mathcal{S}_{+}^{n}, y \in \mathbb{R}^{m}\right\},
$$

where $H$ is a given symmetric positive semidefinite matrix. Suppose $H=R^{T} R$ is the Cholesky factorization. Then the above problem can be rewritten as follows:

$$
\max _{y, Z}\left\{b^{T} y-t:\|R y\|^{2} \leq t, \mathcal{A}_{s}^{T} y+z_{s}=c_{s}, z_{s} \in \mathcal{S}_{+}^{n}, y \in \mathbb{R}^{m}\right\} .
$$

It can in turn be expressed as a standard SQLP in dual form as follows:

$$
\begin{array}{ll}
\max & {[-1 ; b]^{T}[t ; y]} \\
\text { s.t. } & {\left[\begin{array}{cc}
-1 / 2 & 0 \\
-1 / 2 & 0 \\
0 & R
\end{array}\right][t ; y]+z_{q}=\left[\begin{array}{c}
1 / 2 \\
-1 / 2 \\
0
\end{array}\right]} \\
& {\left[0, \mathcal{A}_{s}^{T}\right][t ; y]+z_{s}=c_{s},} \\
& z_{q} \in K_{q}^{m+1}, z_{s} \in \mathcal{S}_{+}^{n}, y \in \mathbb{R}^{m} .
\end{array}
$$

\subsection{An example from distance geometry}

Consider a graph $G=(V, \mathcal{E}, D)$ where $V=\{1,2, \ldots, n\}, \mathcal{E}$, and $D=\left(d_{i j}\right)$ denote the nodes, edges, and associated weight matrix on the edges, respectively. The problem is to find points $x_{1}, \ldots, x_{n}$ in $\mathbb{R}^{p}$ (for some $p$ ) such that the pairwise Euclidean 
distance between $x_{i}$ and $x_{j}$ is as close as possible to $d_{i j}$ if $(i, j) \in \mathcal{E}$. The associated minimization problem is the following:

$$
\min \left\{\sum_{(i, j) \in \mathcal{E}}\left|\left\|x_{i}-x_{j}\right\|^{2}-d_{i j}^{2}\right|: X:=\left[x_{1}, \ldots, x_{n}\right] \in \mathbb{R}^{p \times n}\right\} .
$$

By noting that $\left\|x_{i}-x_{j}\right\|^{2}=e_{i j}^{T} X^{T} X e_{i j}$, where $e_{i j}=e_{i}-e_{j}$, the above problem can equivalently be written as $\min \left\{\sum_{(i, j) \in \mathcal{E}}\left|\left\langle e_{i j} e_{i j}^{T}, Y\right\rangle-d_{i j}^{2}\right|: Y=X^{T} X, X \in \mathbb{R}^{p \times n}\right\}$. One of the SDP relaxations of the above problem is $\min \left\{\sum_{(i, j) \in \mathcal{E}}\left|\left\langle e_{i j} e_{i j}^{T}, Y\right\rangle-d_{i j}^{2}\right|\right.$ : $\left.Y \in \mathcal{S}_{+}^{n}\right\}$, where the corresponding problem in standard form is given by

$\min \left\{\sum_{(i, j) \in \mathcal{E}} \alpha_{i j}^{+}+\alpha_{i j}^{-}:\left\langle e_{i j} e_{i j}^{T}, Y\right\rangle-\alpha_{i j}^{+}+\alpha_{i j}^{-}=d_{i j}^{2}, \alpha_{i j}^{+}, \alpha_{i j}^{-} \geq 0 \forall(i, j) \in \mathcal{E}, Y \in \mathcal{S}_{+}^{n}\right\}$.

Let $m=|\mathcal{E}|$. The SQLP data can be coded as follows:

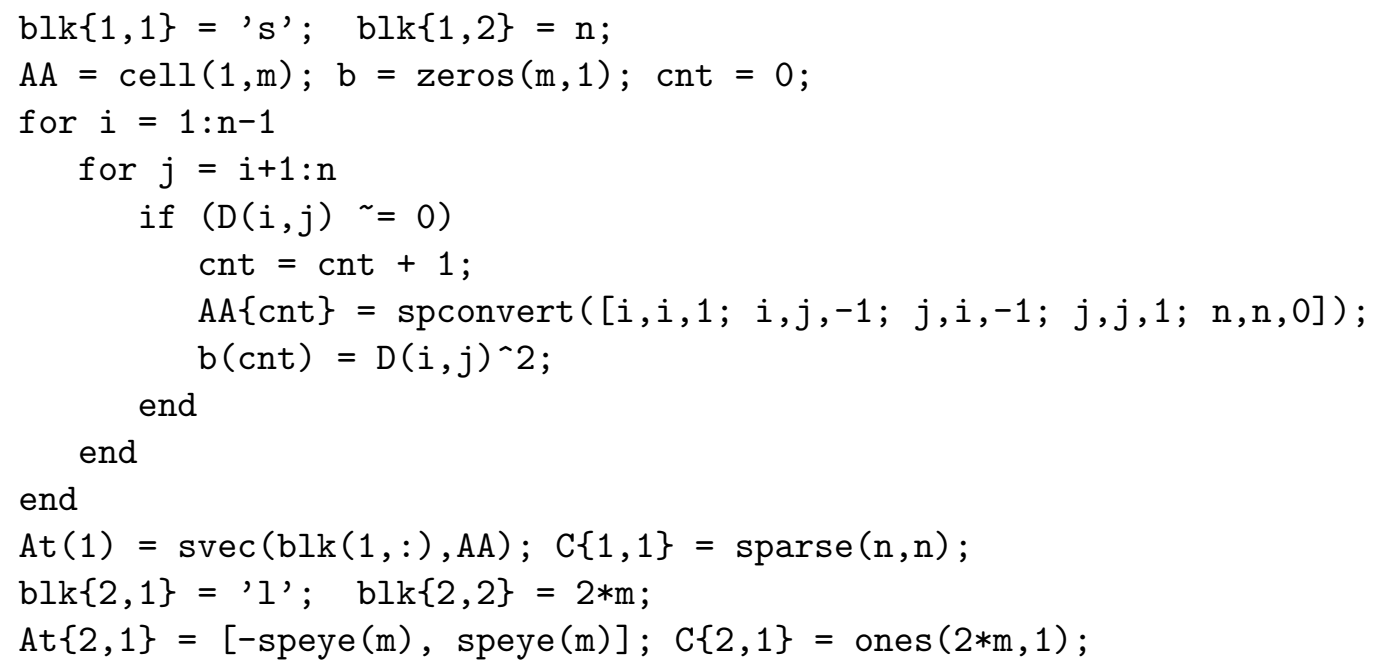

\subsection{Norm minimization problem with complex data}

Let $B_{0}, \ldots B_{m}$ be $p \times q$ matrices that are possibly complex. Consider the norm minimization problem:

$$
\min \left\{t:\left\|\sum_{k=1}^{m} x_{k} B_{k}+B_{0}\right\|_{2} \leq t, x \in \mathbb{C}^{m}, t \in \mathbb{R}\right\},
$$

where $\|\cdot\|_{2}$ denotes the matrix 2-norm. The problem above can equivalently be written as follows:

$$
\begin{array}{ll}
\max & -t \\
\text { s.t. } & \sum_{k=1}^{m} x_{k}^{R}\left[\begin{array}{cc}
0 & B_{k} \\
B_{k}^{*} & 0
\end{array}\right]+\sum_{k=1}^{m} x_{k}^{I}\left[\begin{array}{cc}
0 & i B_{k} \\
\left(i B_{k}\right)^{*} & 0
\end{array}\right]-t I \preceq-\left[\begin{array}{cc}
0 & B_{0} \\
B_{0}^{*} & 0
\end{array}\right] .
\end{array}
$$

This is a complex SDP written in the format as in $(D)$. Such a problem can be solve by SDPT3 as follows: 


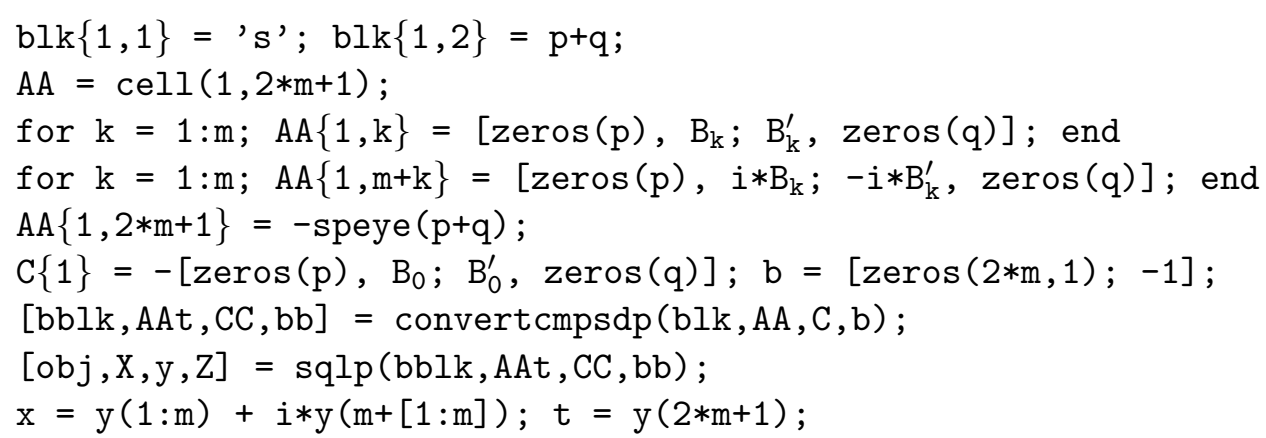

For more details, see the m-file norm_min.m in the subdirectory Examples.

\subsection{Logarithmic Chebyshev approximation problem}

This is an example that contains variables in a rotated cone. The orginal problem [22] is given by $\min _{x \in \mathbb{R}^{m}} \max \left\{\left|\log \left(f_{i}^{T} x\right)-\log \left(d_{i}\right)\right|: i=1, \ldots, p\right\}$, which can equivalently be formulated as: $\min \left\{s: t \leq f_{i}^{T} x / d_{i} \leq s, i=1, \ldots, p, 1 \leq s t\right\}$. Let $F=\left[f_{1}^{T} ; \ldots ; f_{p}^{T}\right]$ and $d=\left[d_{1} ; \ldots ; d_{p}\right]$. The latter problem can be formulated in the following standard dual form:

$$
\begin{aligned}
\max & -s \\
\text { s.t. } & {[F,-d, 0,0][x ; s ; t ; u] \leq 0 } \\
& {[-F, 0, d, 0][x ; s ; t ; u] \leq 0 } \\
& -\left[0,-I_{3}\right][x ; s ; t ; u] \in K_{r}^{3} \\
& {[0,0,0,1][x ; s ; t ; u]=\sqrt{2} }
\end{aligned}
$$

The corresponding data for SDPT3 can be coded as follows:

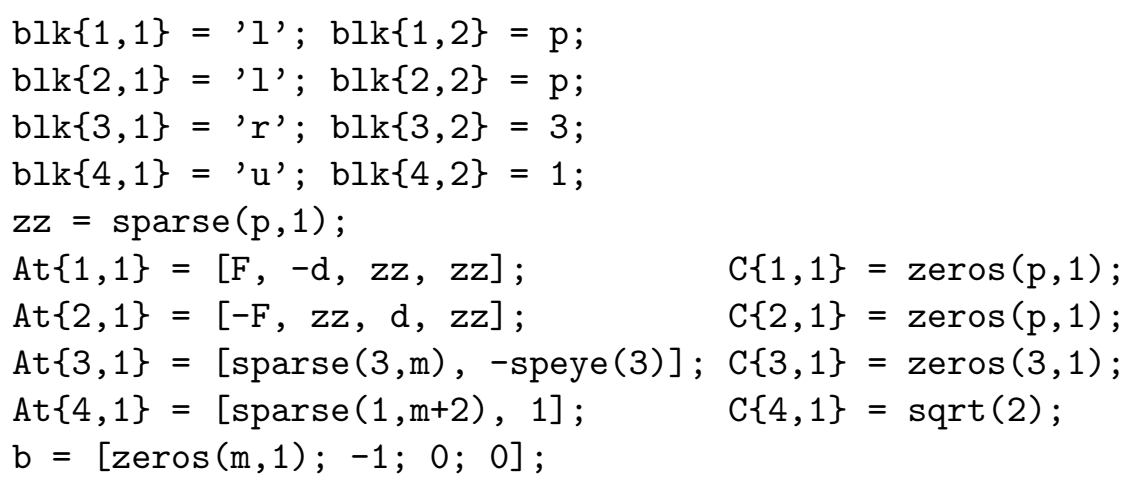

For more details, see the m-file logchebyRcone.m in the subdirectory Examples. 


\subsection{Maximizing the geometric mean of affine functions}

Another example with rotated cone variables comes from maximizing the geometric mean of nonnegative affine functions [22]:

$$
\begin{array}{cl}
\max _{x \in \mathbb{R}^{m}} & \Pi_{i=1}^{4}\left(a_{i}^{T} x+b_{i}\right)^{1 / 4} \\
\text { s.t. } & a_{i}^{T} x+b_{i} \geq 0, i=1, \ldots, 4 .
\end{array}
$$

The above can be reformulated as the following SQLP problem with rotated cone constraints: $\max \left\{\begin{array}{ll}t_{3}: y_{i}=a_{i}^{T} x+b_{i}, y_{i} \geq 0, i=1, \ldots, 4, \quad & \left(\sqrt{2} t_{1}\right)^{2} \leq 2 y_{1} y_{2},\left(\sqrt{2} t_{2}\right)^{2} \leq 2 y_{3} y_{4}, \\ & \left(\sqrt{2} t_{3}\right)^{2} \leq 2 t_{1} t_{2}\end{array}\right\}$.

The corresponding standard (dual) form is as follows:

$$
\begin{aligned}
& \max t_{3} \\
& \text { s.t. }\left[\begin{array}{cccc}
a_{1}^{T} & 0 & 0 & 0 \\
a_{2}^{T} & 0 & 0 & 0 \\
0 & \sqrt{2} & 0 & 0 \\
a_{3}^{T} & 0 & 0 & 0 \\
a_{4}^{T} & 0 & 0 & 0 \\
0 & 0 & \sqrt{2} & 0 \\
0 & 1 & 0 & 0 \\
0 & 0 & 1 & 0 \\
0 & 0 & 0 & \sqrt{2} \\
a_{1}^{T} & 0 & 0 & 0 \\
a_{3}^{T} & 0 & 0 & 0
\end{array}\right]\left[\begin{array}{c}
x \\
t_{1} \\
t_{2} \\
t_{3}
\end{array}\right]+\left[\begin{array}{c}
b_{1} \\
b_{2} \\
0 \\
b_{3} \\
b_{4} \\
0 \\
0 \\
0 \\
0 \\
b_{1} \\
b_{3}
\end{array}\right] \in K_{r}^{3} \times K_{r}^{3} \times K_{r}^{3} \times \mathbb{R}_{+}^{2} \text {. }
\end{aligned}
$$

\section{$5 \quad$ Implementation details}

The main step at each iteration of our algorithms is the computation of the search direction $(\Delta x, \Delta y, \Delta z)$ from the symmetrized Newton equation with respect to some invertible block diagonal scaling matrix that is usually chosen as a function of the current iterate $x, z$.

\subsection{The HKM search direction}

Let

$$
J_{i}^{q}=\left[\begin{array}{cc}
1 & 0 \\
0 & -I_{q_{i}-1}
\end{array}\right]
$$


For the choice of the HKM scaling matrix [16, 20, 24, 25, 38], the search direction $(\Delta x, \Delta y, \Delta z)$ is obtained from the following system of equations:

$$
\begin{aligned}
\mathcal{A}^{s}\left(\Delta x^{s}\right)+A^{q}\left(\Delta x^{q}\right)+A^{l} \Delta x^{l}+A^{u} \Delta x^{u} & =R_{p}:=b-\mathcal{A}^{s}\left(x^{s}\right)-A^{q}\left(x^{q}\right)-A^{l} x^{l}-A^{u} x^{u} \\
\left(\mathcal{A}^{s}\right)^{T} \Delta y+\Delta z^{s} & =R_{d}^{s}:=c^{s}-z^{s}-\left(\mathcal{A}^{s}\right)^{T} y \\
\left(A^{q}\right)^{T} \Delta y+\Delta z^{q} & =R_{d}^{q}:=c^{q}-z^{q}-\left(A^{q}\right)^{T} y \\
\left(A^{l}\right)^{T} \Delta y+\Delta z^{l} & =R_{d}^{l}:=c^{l}-z^{l}-\left(A^{l}\right)^{T} y \\
\left(A^{u}\right)^{T} \Delta y & =R_{d}^{u}:=c^{u}-\left(A^{u}\right)^{T} y \\
\Delta x^{s}+H^{s}\left(\Delta z^{s}\right) & =R_{c}^{s}:=\left(\max \left\{\sigma \mu(x, z), \nu_{j}^{s}\right\}\left(z_{j}^{s}\right)^{-1}-x_{j}^{s}\right)_{j=1}^{n_{s}} \\
\Delta x^{q}+H^{q}\left(\Delta z^{q}\right) & =R_{c}^{q}:=\left(\max \left\{\sigma \mu(x, z), \nu_{i}^{q}\right\}\left(z_{i}^{q}\right)^{-1}-x_{i}^{q}\right)_{i=1}^{n_{q}} \\
\Delta x^{l}+H^{l}\left(\Delta z^{l}\right) & =R_{c}^{l}:=\left(\max \left\{\sigma \mu(x, z), \nu_{k}^{l}\right\}\left(z_{k}^{l}\right)^{-1}-x_{k}^{l}\right)_{k=1}^{n_{l}},
\end{aligned}
$$

where $\sigma \in(0,1)$ is the centering parameter; $\left(z_{j}^{s}\right)^{-1}$ and $\left(z_{k}^{l}\right)^{-1}$ have the usual meaning, and $\left(z_{i}^{q}\right)^{-1}:=J_{i}^{q} z_{i}^{q} / \gamma\left(z_{i}^{q}\right)^{2}$. In the above,

$$
\begin{gathered}
H^{s}\left(\Delta z^{s}\right)=\left(H_{j}^{s}\left(\Delta z_{j}^{s}\right):=\frac{1}{2}\left(\left(z_{j}^{s}\right)^{-1} \Delta z_{j}^{s} x_{j}^{s}+x_{j}^{s} \Delta z_{j}^{s}\left(z_{j}^{s}\right)^{-1}\right)\right)_{j=1}^{n_{s}}, \\
H^{q}\left(\Delta z^{q}\right)=\left(H_{i}^{q}\left(\Delta z_{i}^{q}\right):=-\frac{\left\langle x_{i}^{q}, z_{i}^{q}\right\rangle}{\gamma\left(z_{i}^{q}\right)^{2}} J_{i}^{q} \Delta z_{i}^{q}+x_{i}^{q}\left(\left(z_{i}^{q}\right)^{-1}\right)^{T} \Delta z_{i}^{q}+\left(z_{i}^{q}\right)^{-1}\left(x_{i}^{q}\right)^{T} \Delta z_{i}^{q}\right)_{i=1}^{n_{q}}, \\
H^{l}\left(\Delta z^{l}\right)=\operatorname{Diag}\left(x^{l}\right) \operatorname{Diag}\left(z^{l}\right)^{-1} \Delta z^{l} .
\end{gathered}
$$

The search direction can be computed via a Schur complement equation as follows (the reader is referred to [1] and [30] for details). First compute $\Delta y$ from the Schur complement equation

$$
\underbrace{\left[\begin{array}{cc}
M & A^{u} \\
\left(A^{u}\right)^{T} & 0
\end{array}\right]}_{\mathcal{M}}\left[\begin{array}{c}
\Delta y \\
\Delta x^{u}
\end{array}\right]=\left[\begin{array}{c}
h \\
R_{d}^{u}
\end{array}\right]
$$

where

$$
\begin{aligned}
M & =\sum_{j=1}^{n_{s}} \underbrace{\mathcal{A}_{j}^{s} H_{j}^{s}\left(\mathcal{A}_{j}^{s}\right)^{T}}_{M_{j}^{s}}+\sum_{i=1}^{n_{q}} \underbrace{A_{i}^{q} H_{i}^{q}\left(A_{i}^{q}\right)^{T}}_{M_{i}^{q}}+\underbrace{A^{l} H^{l}\left(A^{l}\right)^{T}}_{M^{l}} \\
h & =R_{p}+\mathcal{A}^{s}\left(H^{s}\left(R_{d}^{s}\right)-R_{c}^{s}\right)+A^{q}\left(H^{q}\left(R_{d}^{q}\right)-R_{c}^{q}\right)+A^{l}\left(H^{l}\left(R_{d}^{l}\right)-R_{c}^{l}\right) .
\end{aligned}
$$

(The notation in (14) should be interpreted as follows: the $k$ th columns of $M_{j}^{s}$ and $M_{i}^{q}$ are $\mathcal{A}_{j}^{s} H_{j}^{s}\left(a_{j, k}^{s}\right)$ and $A_{i}^{q} H_{i}^{q}\left(a_{i, k}^{q}\right)$, with $a_{i, k}^{q}$ the $k$ th column of $\left(A_{i, k}^{q}\right)^{T}$. Note that 
the matrices $M_{j}^{s}, M_{i}^{q}, M^{l}$ are all symmetric positive definite.) Then compute $\Delta x$ and $\Delta z$ from the equations

$$
\begin{array}{lll}
\Delta z^{s}=R_{d}^{s}-\left(\mathcal{A}^{s}\right)^{T} \Delta y, & \Delta z^{q}=R_{d}^{q}-\left(A^{q}\right)^{T} \Delta y, & \Delta z^{l}=R_{d}^{l}-\left(A^{l}\right)^{T} \Delta y \\
\Delta x^{s}=R_{c}^{s}-H^{s}\left(\Delta z^{s}\right), & \Delta x^{q}=R_{c}^{q}-H^{q}\left(\Delta z^{q}\right), & \Delta x^{l}=R_{c}^{l}-H^{l}\left(\Delta z^{l}\right) .
\end{array}
$$

\subsection{The NT search direction}

The user also has the choice of using the NT direction [28, 25, 38]. Let $w_{j}^{s}$ be the unique positive definite matrix such that $w_{j}^{s} z_{j}^{s} w_{j}^{s}=x_{j}^{s}$. Let

$$
\omega_{i}^{q}=\sqrt{\frac{\gamma\left(z_{i}^{q}\right)}{\gamma\left(x_{i}^{q}\right)}}, \quad \xi_{i}^{q}=\frac{1}{\omega_{i}^{q}} z_{i}^{q}+\omega_{i}^{q} J_{i}^{q} x_{i}^{q}, \quad t_{i}^{q}=\frac{\sqrt{2}}{\omega_{i}^{q} \gamma\left(\xi_{i}^{q}\right)} J_{i}^{q} \xi_{i}^{q} .
$$

In this case, the search direction $(\Delta x, \Delta y, \Delta z)$ satisfies the same system as in (11) except that $H^{s}$ and $H^{q}$ are replaced by

$$
\begin{gathered}
H^{s}\left(\Delta z^{s}\right)=\left(H_{j}^{s}\left(\Delta z_{j}^{s}\right):=w_{j}^{s} \Delta z_{j}^{s} w_{j}^{s}\right)_{j=1}^{n_{s}}, \\
H^{q}\left(\Delta z^{q}\right)=\left(H_{i}^{q}\left(\Delta z_{i}^{q}\right):=-\frac{1}{\left(\omega_{i}^{q}\right)^{2}} J_{i}^{q} \Delta z_{i}^{q}+t_{i}^{q}\left(t_{i}^{q}\right)^{T} \Delta z_{i}^{q}\right)_{i=1}^{n_{q}} .
\end{gathered}
$$

\subsection{Choice of search direction}

The current version of the code allows only two search directions, HKM and NT. In older versions, version 2.3 or earlier, we also implemented the AHO direction of Alizadeh, Haeberly, and Overton [1] and the GT direction [32], where both directions tend to give more accurate results, but these are uncompetitive when the problems are of large scale.

For the HKM and NT search directions, our computational experience on problems tested in Section 8 is that the HKM direction is almost universally faster than NT on problems with semidefinite blocks, especially for sparse problems with large semidefinite blocks. The reason that the latter is slower is because computing the NT scaling matrix $w_{j}^{s}$ requires a full eigenvalue decomposition. This computation can dominate the work at each interior-point iteration when the problem is sparse.

The NT direction, however, was faster on sparse SOCP problems. The reason for this behavior is not hard to understand. By comparing the formulae for $H_{i}^{q}$ for the HKM and NT directions in (12) and (16), it is clear that more computation is required to assemble the Schur complement matrix and more low-rank updating is necessary for the former direction.

\subsection{Computation of the Schur complement matrix}

Generally, the most expensive part in each iteration of Algorithm IPC lies in the computation and factorization of the Schur complement matrix $M$ defined in (13). 
And this depends critically on the size and density of $M$. Note that the density of this matrix depends on two factors: (i) The density of $\mathcal{A}^{s}, A^{q}$, and $A^{l}$, and (ii) any additional fill-in introduced because of the terms $H^{s}, H^{q}$, and $H^{l}$ in (14).

\subsubsection{Semidefinite blocks}

For problems with semidefinite blocks, the contribution by the $j$ th semidefinite block to $M$ is given by $M_{j}^{s}:=\mathcal{A}_{j}^{s} H_{j}^{s}\left(\mathcal{A}_{j}^{s}\right)^{T}$. The matrix $M_{j}^{s}$ is generally dense even if $A_{j}^{s}$ is sparse. The computation of each entry of $M_{j}^{s}$ involves matrix products, which has the form

$$
\left(M_{j}^{s}\right)_{\alpha \beta}= \begin{cases}\left\langle a_{j, \alpha}^{s}, x_{j}^{s} a_{j, \beta}^{s}\left(z_{j}^{s}\right)^{-1}\right\rangle & \text { for the HKM direction. } \\ \left\langle a_{j, \alpha}^{s}, w_{j}^{s} a_{j, \beta}^{s} w_{j}^{s}\right\rangle & \text { for the NT direction. }\end{cases}
$$

This computation can be very expensive if it is done naively without properly exploiting the sparsity that is generally present in the constraint matrices in $\mathcal{A}_{j}^{s}$. In our earlier papers [30,34], we discussed briefly how sparsity of $\mathcal{A}_{j}^{s}$ is exploited in our implementation by following the ideas presented by Fujisawa, Kojima, and Nakata in [13]. Further details on the efficient implementation of these ideas are given in [39].

When the constraint matrices have low-rank structures as described in Section 2.2 , we can also compute the element $\left(M_{j}^{S}\right)_{\alpha, \beta}$ as follows:

$$
\left(M_{j}^{s}\right)_{\alpha \beta}=\operatorname{Tr}\left(\widetilde{V}_{\beta}^{T} V_{\alpha} D_{\alpha} V_{\alpha}^{T} \widehat{V}_{\beta} D_{\beta}\right),
$$

where $\widetilde{V}_{\beta}=x_{j}^{s} V_{\beta}$, and $\widehat{V}_{\beta}=\left(z_{j}^{s}\right)^{-1} V_{\beta}$ if the HKM direction is used; and $\widetilde{V}_{\beta}=$ $\widehat{V}_{\beta}=w_{j}^{s} V_{\beta}$ if the NT direction is used. Assume for simplicity that all the constraint matrices associated with the $j$ th semidefinite block are dense and low-rank, i.e., $p=0$ in Section 2.2. Suppose that the matrices $\widetilde{V}_{k}, \widehat{V}_{k}, k=1, \ldots, m$, are pre-computed (at the cost of $\Theta\left(s_{j}^{2} \sum_{k=1}^{m} r_{j, k}\right)$ flops $)$. Then it would take an additional $\Theta\left(s_{j}\left(\sum_{k=1}^{m} r_{j, k}\right)^{2}\right)$ flops to compute $M_{j}^{s}$ since each element $\left(M_{j}^{s}\right)_{\alpha \beta}$ can be computed at $\Theta\left(s_{j} r_{j, \alpha} r_{j, \beta}\right)$ flops. In contrast, without exploiting the low-rank structures, it would take $\Theta\left(s_{j}^{3} m\right)+$ $\Theta\left(s_{j}^{2} m^{2}\right)$ flops to compute $M_{j}^{s}$. If the average rank of the constraint matrices is $r$, then the latter complexity is $\Theta\left(s_{j} / r^{2}\right)$ times larger than the former of $\Theta\left(s_{j}^{2} m r\right)+$ $\Theta\left(s_{j} m^{2} r^{2}\right)$. Thus it is definitely advantageous to exploit low-rank structures.

As an example, we generated a random SDP with low rank structure using the m-file randlowranksdp.m described in Section 2.2 with $n=200$ and $m=1000$; the solver sqlp.m ran about 6 times faster when the low-rank structure was exploited.

\subsubsection{Quadratic and linear blocks}

For the linear block, $H^{l}$ is a diagonal matrix and it does not introduce any additional fill-in. This matrix does, however, affect the conditioning of the Schur complement matrix.

From equation (14), it is easily shown that the contribution of the quadratic blocks 
to the matrix $M$ is given by

$$
M_{i}^{q}= \begin{cases}-\frac{\left\langle x_{i}^{q}, z_{i}^{q}\right\rangle}{\gamma^{2}\left(z_{i}^{q}\right)} A_{i}^{q} J_{i}^{q}\left(A_{i}^{q}\right)^{T}+u_{i}^{q}\left(v_{i}^{q}\right)^{T}+v_{i}^{q}\left(u_{i}^{q}\right)^{T}, & \text { for HKM direction } \\ -\frac{1}{\left(\omega_{i}^{q}\right)^{2}} A_{i}^{q} J_{i}^{q}\left(A_{i}^{q}\right)^{T}+w_{i}^{q}\left(w_{i}^{q}\right)^{T} & \text { for NT direction }\end{cases}
$$

where $u_{i}^{q}=A_{i}^{q} x_{i}^{q}, v_{i}^{q}=A_{i}^{q}\left(z_{i}^{q}\right)^{-1}, w_{i}^{q}=A_{i}^{q} t_{i}^{q}$.

In the rest of this subsection, we focus our discussion on the HKM direction, but the same holds true for the NT direction.

The appearance of the outer-product terms in the equation above is potentially alarming. If the vectors $u_{i}^{q}, v_{i}^{q}$ are dense, then even if $A_{i}^{q}$ is sparse, the corresponding matrix $M_{i}^{q}$, and hence the Schur complement matrix $M$, will be dense. A direct factorization of the resulting dense matrix will be very expensive for even moderately large $m$.

The density of the matrix $M_{i}^{q}$ depends largely on the particular problem structure. When the problem has many small quadratic blocks, it is often the case that each block appears in only a small fraction of the constraints. In this case, all $A_{i}^{q}$ matrices are sparse and the vectors $u_{i}^{q}$ and $v_{i}^{q}$ turn out to be sparse vectors for each $i$. Consequently, the matrices $M_{i}^{q}$ remain relatively sparse for these problems. As a result, $M$ is also sparse and it can be factorized directly with reasonable cost. This behavior is typical for all nql and qssp problems from the DIMACS library.

The situation is drastically different for problems where one of the quadratic blocks, say the $i$ th block, is large. For such problems the vectors $u_{i}^{q}, v_{i}^{q}$ are typically dense, and therefore, $M_{i}^{q}$ is likely be a dense matrix even if the data $A_{i}^{q}$ is sparse. However, observe that $M_{i}^{q}$ is a rank-two perturbation of a sparse matrix when $A_{i}^{q}\left(A_{i}^{q}\right)^{T}$ is sparse. In such a situation, it is advantageous to use the dense-column handling technique described in Section 5.7 to reduce the computational cost in solving (13). This approach helps tremendously on the scheduling problems from the DIMACS library.

To apply the dense-column handling technique, we need to modify the sparse portion of the matrix $M_{i}^{q}$ slightly. Since the diagonal matrix $-J_{i}$ has a negative component, the matrix $-A_{i}^{q} J_{i}^{q}\left(A_{i}^{q}\right)^{T}$ need not be a positive definite matrix, and therefore the Cholesky factorization of the sparse portion of $M_{i}^{q}$ need not exist. To overcome this difficulty, we use the following identity:

$$
M_{i}^{q}=\frac{\left\langle x_{i}^{q}, z_{i}^{q}\right\rangle}{\gamma^{2}\left(z_{i}^{q}\right)} A_{i}^{q}\left(A_{i}^{q}\right)^{T}+u_{i}^{q}\left(v_{i}^{q}\right)^{T}+v_{i}^{q}\left(u_{i}^{q}\right)^{T}-k_{i} k_{i}^{T},
$$

where $k_{i}=\sqrt{2}\left\langle x_{i}^{q}, z_{i}^{q}\right\rangle / \gamma^{2}\left(z_{i}^{q}\right) A_{i}^{q}(:, 1)$. Note that if $A_{i}^{q}$ is a large sparse matrix with a few dense columns, we can also explicitly separate the outer-product terms contributed by these dense columns from the sparse part of $A_{i}^{q}\left(A_{i}^{q}\right)^{T}$ in (18).

\subsection{Solving the Schur complement equation}

The linear system (13) typically becomes more and more ill-conditioned as $\mu(x, z)$ decreases to 0 . Thus iterative refinement is generally recommended to improve the 
accuracy of the computed solution. An even better approach to solve (13) is via a preconditioned symmetric quasi-minimal residual method (PSQMR) [11] with the preconditioner computed based on the following analytical expression of $\mathcal{M}^{-1}$ :

$$
\mathcal{M}^{-1}=\left[\begin{array}{cc}
M^{-1}-M^{-1} A^{u} S^{-1}\left(A^{u}\right)^{T} M^{-1} & M^{-1} A^{u} S^{-1} \\
S^{-1}\left(A^{u}\right)^{T} M^{-1} & -S^{-1}
\end{array}\right],
$$

where $S=\left(A^{u}\right)^{T} M^{-1} A^{u}$. Note that for a given vector $[u ; v], \mathcal{M}^{-1}[u ; v]$ can be evaluated efficiently as follows:

$$
\begin{array}{cl}
\hat{u} & =M^{-1} u \\
t & =S^{-1}\left(\left(A^{u}\right)^{T} \hat{u}-v\right) \\
\mathcal{M}^{-1}[u ; v] & =\left[\hat{u}-M^{-1} A^{u} t ; t\right] .
\end{array}
$$

Thus if the Cholesky factorization of $M$ and that of $S$ are computed, then each evaluation involves solving four triangular linear systems for $M$ and two triangular linear systems for $S$.

We should mention that state-of-the-art Cholesky factorization software is highly developed and optimized. Thus our preference is to solve a linear system via Cholesky factorizations whenever possible. For most SDP problems, the matrix $M$ is typically dense even when the constraint matrices are sparse. In this case, we use the routine chol (based on the LAPACK routine dpotrf) in MATLAB to compute the Cholesky factorization of a dense matrix.

For most sparse SOCP problems, the matrix $M$ is usually sparse after densecolumn handling. Let $M_{s p}$ be the sparse part of $M$ after dense-column handling. In this case, the Cholesky factorization routine chol for a dense matrix is not efficient enough since it does not exploit sparsity. To factorize the sparse matrix $M_{s p}$ more efficiently, we use the sparse cholesky solver cholmod of Davis [5], which is available in MATLAB as chol. In earlier versions, we used a $\mathrm{C}$ translation of the Fortran programs developed by Ng, Peyton, and Liu for sparse Cholesky factorization [26].

The effect of using a sparse Cholesky solver for sparse SOCP problems was dramatic. We observed speed-ups of up to two orders of magnitude. In our implementation, SDPT3 automatically makes a choice between MATLAB's built-in chol routine and the sparse Cholesky solver based on the density of $M$. The cutoff density is specified in the parameter OPTIONS.spdensity.

The approach of solving (13) by the SQMR method with preconditioner (19) works reasonably well if the following conditions are satisfied: (i) the number of columns of $A^{u}$ is small and $A^{u}$ is well-conditioned; (ii) the matrix $M$ is not extremely illconditioned. (iii) the matrix $S$ is not extremely ill-conditioned. However, when these conditions are not satisfied, preconditioning (13) based on (19) may not be advisable because either (a) computing $S$ becomes very expensive due to large number of columns in $A^{u}$, or (b) the computed preconditioner based on (19) is no longer an accurate approximation of $\mathcal{M}^{-1}$. Note that $S$ is typically much more ill-conditioned than $A^{u}$, especially when $A^{u}$ is ill-conditioned. When conditions (i)-(iii) are not 
satisfied, it is advisable to directly use an $L U$ or $L D L^{T}$ factorization of the symmetric indefinite matrix $\mathcal{M}$ to compute an approximation of $\mathcal{M}^{-1}$. As the matrix $\mathcal{M}$ is usually highly ill-conditioned, we observed that the computed solution based on $L U$ factorization is typically more accurate than the one computed based on $L D L^{T}$ factorization. Thus, even though $L U$ is twice as expensive as $L D L^{T}$ factorization when the matrix is dense, we simply use the MATLAB routine lu to compute an $L U$ factorization of $\mathcal{M}$, and use the computed $L U$ factors to precondition the SQMR iterative method used to solve (13).

Again, in our implementation, SDPT3 automatically makes a choice on whether to compute a dense or sparse $L U$ factorization based on the density of $\mathcal{M}$. In the case of sparse $L U$ factorization, SDPT3 uses the UMFPACK package of Davis [6], which is available in MATLAB under the $1 \mathrm{u}$ command. We should mention that we have tested the sparse $L D L^{T}$ factorization of the symmetric matrix $\mathcal{M}$ (when it is a sparse) based on the MA57 routine [7] (available in MATLAB under the ldl command) of the Harwell subroutine library. But we have found that it is not as efficient as the sparse $L U$ factorization based on the UMFPACK package when the matrix is highly ill-conditioned. More importantly, the computed solution based on sparse $L D L^{T}$ factorization is often not as accurate as the one computed based on sparse $L U$ factorization.

\subsection{Internal handling of unrestricted blocks}

As mentioned in the last sub-section, solving the symmetric indefinite system (13) can potentially be very expensive when $A^{u}$ is ill-conditioned or has a large number of columns because computing the sparse $L U$ factorization of a sparse matrix can be much more costly than that for a symmetric positive definite matrix of the same order. It is possible to avoid the need to solve a symmetric indefinite system if we reformulate the equality constraint in $(D)$ as

$$
\begin{gathered}
\left(A^{u}\right)^{T} y+z_{+}^{u}=c^{u}, \quad z_{+}^{u} \geq 0 \\
-\left(A^{u}\right)^{T} y+z_{-}^{u}=-c^{u}, \quad z_{-}^{u} \geq 0,
\end{gathered}
$$

with the corresponding primal variable $x^{u}$ expressed as

$$
x^{u}=x_{+}^{u}-x_{-}^{u}, \quad x_{+}^{u}, x_{-}^{u} \geq 0 .
$$

In this case, the system (13) is replaced by

$$
\left(M+A^{u} \operatorname{Diag}\left(x_{+}^{u}\right) \operatorname{Diag}\left(z_{+}^{u}\right)^{-1}\left(A^{u}\right)^{T}+A^{u} \operatorname{Diag}\left(x_{-}^{u}\right) \operatorname{Diag}\left(z_{-}^{u}\right)^{-1}\left(A^{u}\right)^{T}\right) \Delta y=\operatorname{rhs}
$$

where rhs denotes the right-hand-side vector. Notice that in contrast to (13), the coefficient matrix is now symmetric positive definite.

But such a reformulation is not without difficulties. In fact, the variables $x_{+}^{u}, x_{-}^{u}$ tend to become very large and $z_{+}^{u}, z_{-}^{u}$ tend to become extremely small as the interiorpoint iteration progresses, and this generally makes the component matrices, $A^{u} \operatorname{Diag}\left(x_{+}^{u}\right) \operatorname{Diag}\left(z_{+}^{u}\right)^{-1}\left(A^{u}\right)^{T}$ and $A^{u} \operatorname{Diag}\left(x_{-}^{u}\right) \operatorname{Diag}\left(z_{-}^{u}\right)^{-1}\left(A^{u}\right)^{T}$, extremely ill-conditioned. 
Fortunately, the following heuristic to modify the vectors $x_{+}^{u}, x_{-}^{u}$ can typically ameliorate such an ill-conditioning problem:

$$
x_{+}^{u}:=x_{+}^{u}-0.8 \min \left(x_{+}^{u}, x_{-}^{u}\right), \quad x_{-}^{u}:=x_{-}^{u}-0.8 \min \left(x_{+}^{u}, x_{-}^{u}\right) .
$$

This modification does not change the original variable $x^{u}$ but does slow down the growth of $x_{+}^{u}, x_{-}^{u}$. After these modified vectors have been obtained, we also add positive perturbations to the vectors $z_{+}^{u}, z_{-}^{u}$. Such a modification in $z_{+}^{u}, z_{-}^{u}$ ensures that they approach 0 at the same rate as $\mu$, and thus prevents the dual problem $(D)$ from approaching the equality constraint too closely prematurely.

For computational efficiency, in the current implementation of SDPT3, we always reformulate an unrestricted vector by the difference of two non-negative vectors.

\subsection{Dense-column handling}

Here we describe our technique to handle dense columns when $M$ is a low-rank perturbation of a sparse matrix. In such a case, the Schur complement matrix $M$ can be written in the form

$$
M=M_{s p}+U D U^{T}
$$

where $M_{s p}$ is a sparse symmetric positive semidefinite matrix, $U$ has only a few columns, and $D$ is a non-singular matrix. If $M_{s p}$ is positive definite, then we can solve (13) by solving a slightly larger but sparse linear system as follows. Let $\lambda=D U^{T} \Delta y$. It is easy to show that (13) is equivalent to the following linear system:

$$
\left[\begin{array}{ccc}
M_{s p} & A^{u} & U \\
\left(A^{u}\right)^{T} & 0 & 0 \\
U^{T} & 0 & -D^{-1}
\end{array}\right]\left[\begin{array}{c}
\Delta y \\
\Delta x^{u} \\
\lambda
\end{array}\right]=\left[\begin{array}{c}
h \\
R_{d}^{u} \\
0
\end{array}\right] .
$$

We can use the same method described in Section 5.5 to solve (21).

\subsection{User supplied routine to compute Schur complement matrix}

The current version of SDPT3 allows the user to supply specialized routines to compute the Schur complement matrices $M_{j}^{s}$ corresponding to the semidefinite blocks.

The specialized routine to compute $M_{j}^{s}$ should have first line that look like:

function schurmat $=\operatorname{schurfun}_{-} j$ th $\left(U, V\right.$, schurfun $_{-} j$ th_par $)$;

where the input arguments $\mathrm{U}$ and $\mathrm{V}$ should correspond to $x_{j}^{s}$ and $\left(z_{j}^{s}\right)^{-1}$ if the HKM direction is used; and they should correspond to the NT scaling matrix $w_{j}^{s}$ if the NT direction is used. The third optional argument schurfun_jth_par can be a structure array that stores the parameters needed inside the function schurfun_jth.m.

The user can tell SDPT3 to use the specialized routine by setting the $L \times 1$ cell array OPTIONS.schurfun as follows: set OPTIONS.schurfun $\{j\}=\operatorname{schurfun}_{-}$th if $M_{j}^{s}$ 
is to be computed by the specialized routine coded in the function schurfun jth $_{\text {.m; }}$ otherwise set OPTIONS.schurfun $\{j\}=[]$. If the function schurfun $-j$ th.m requires some parameters, then the $L \times 1$ cell array OPTIONS.schurfun_par must also be set correspondingly as follows: set OPTIONS.schurfun_par $\{j\}=\operatorname{schurfun}_{-} j$ th_par; otherwise set OPTIONS.schurfun_par $\{j\}=[]$.

Below is an example on we how use the specialized routine mcpschur.m in the subdirectory Examples to compute the Schur complement matrix when solving the SDP problem mcp250-1. dat-s.

$\gg[\mathrm{blk}, \mathrm{At}, \mathrm{C}, \mathrm{b}]=\mathrm{read} \_\mathrm{sdpa}\left({ }^{\prime} . / \mathrm{sdplib} / \mathrm{mcp} 250-1\right.$. dat-s' $)$;

$>$ OPTIONS.schurfun $\{1\}=$ 'mcpschur';

> $[o b j, X, y, Z]=\operatorname{sqlp}(b l k, A t, C, b$, OPTIONS) ;

In the above example, there is no need to set the cell array OPTIONS.schurfun_par since the function mcpschur.m does not need any additional parameters.

\subsection{Step-length computation}

Once a direction $\Delta x$ is computed, a full step will not be allowed if $x+\Delta x$ violates the conic constraints. Thus, the next iterate must take the form $x+\alpha \Delta x$ for an appropriate choice of the step-length $\alpha$. In this subsection, we discuss an efficient strategy to compute the step-length $\alpha$.

For semidefinite blocks, it is straightforward to verify that, for the $j$ th block, the maximum allowed step-length that can be taken without violating the positive semidefiniteness of the matrix $x_{j}^{s}+\alpha_{j}^{s} \Delta x_{j}^{s}$ is given as follows:

$$
\alpha_{j}^{s}= \begin{cases}\frac{-1}{\lambda_{\min }\left(\left(x_{j}^{s}\right)^{-1} \Delta x_{j}^{s}\right)}, & \text { if the minimum eigenvalue } \lambda_{\min } \text { is negative } \\ \infty & \text { otherwise. }\end{cases}
$$

If the computation of eigenvalues necessary in $\alpha_{j}^{s}$ above becomes expensive, then we resort to finding an approximation of $\alpha_{j}^{s}$ by estimating extreme eigenvalues using Lanczos iterations [31]. This approach is quite accurate in general and represents a good trade-off between the computational effort versus quality of the resulting stepsizes.

For quadratic blocks, the largest step-length $\alpha_{i}^{q}$ that keeps the next iterate feasible with respect to the $i$ th quadratic cone can be computed as follows. Let

$$
a_{i}=\gamma\left(\Delta x_{i}^{q}\right)^{2}, \quad b_{i}=\left\langle\Delta x_{i}^{q}, J_{i}^{q} x_{i}^{q}\right\rangle, \quad c_{i}=\gamma\left(x_{i}^{q}\right)^{2}, \quad d_{i}=b_{i}^{2}-a_{i} c_{i},
$$

where $J_{i}^{q}$ is the matrix defined in (10). We want the largest positive $\bar{\alpha}$ for which $a_{i} \alpha^{2}+2 b_{i} \alpha+c_{i}>0$ for all smaller positive $\alpha$ 's, which is given by

$$
\alpha_{i}^{q}= \begin{cases}\frac{-b_{i}-\sqrt{d_{i}}}{a_{i}} & \text { if } a_{i}<0 \text { or } b_{i}<0, a_{i} \leq b_{i}^{2} / c_{i} \\ \frac{-c_{i}}{2 b_{i}} & \text { if } a_{i}=0, b_{i}<0 \\ \infty & \text { otherwise. }\end{cases}
$$


For the linear block, the maximum allowed step-length $\alpha_{k}^{l}$ for the $k$ th component is given by

$$
\alpha_{k}^{l}= \begin{cases}\frac{-x_{k}^{l}}{\Delta x_{k}^{l}}, & \text { if } \Delta x_{k}^{l}<0 \\ \infty & \text { otherwise. }\end{cases}
$$

Finally, an appropriate step-length $\alpha$ that can be taken in order for $x+\alpha \Delta x$ to satisfy all the conic constraints takes the form

$$
\alpha=\min \left(1, \bar{\gamma} \min _{1 \leq j \leq n_{s}} \alpha_{j}^{s}, \bar{\gamma} \min _{1 \leq i \leq n_{q}} \alpha_{i}^{q}, \bar{\gamma} \min _{1 \leq k \leq n_{l}} \alpha_{k}^{l}\right),
$$

where $\bar{\gamma}$ (known as the step-length parameter) is typically chosen to be a number slightly less than 1 , say 0.98 , to ensure that the next iterate $x+\alpha \Delta x$ stays strictly in the interior of all the cones.

For the dual direction $\Delta z$, we let the analog of $\alpha_{j}^{s}, \alpha_{i}^{q}$ and $\alpha_{k}^{l}$ be $\beta_{j}^{s}, \beta_{i}^{q}$ and $\beta_{k}^{l}$, respectively. Similar to the primal direction, the step-length that can be taken by the dual direction $\Delta z$ is given by

$$
\beta=\min \left(1, \bar{\gamma} \min _{1 \leq j \leq n_{s}} \beta_{j}^{s}, \bar{\gamma} \min _{1 \leq i \leq n_{q}} \beta_{i}^{q}, \bar{\gamma} \min _{1 \leq k \leq n_{l}} \beta_{k}^{l}\right)
$$

\section{$6 \quad$ Homogeneous self-dual model}

In the current version of SDPT3, we have implemented algorithms analogous to Algorithm IPC in Appendix A to solve the following 3-parameter homogeneous self-dual (HSD) model [41] of $(P)$ and $(D)$ when the problems have no logarithmic terms or unrestricted variables:

$$
\begin{aligned}
\left(P_{H}\right) \quad \min & \bar{\alpha} \theta \\
\text { s.t. } & {\left[\begin{array}{cccc}
0 & -\mathcal{A} & b & -\bar{b} \\
\mathcal{A}^{T} & 0 & -c & \bar{c} \\
-b^{T} & c^{T} & 0 & -\bar{g} \\
\bar{b}^{T} & -\bar{c}^{T} & \bar{g} & 0
\end{array}\right]\left[\begin{array}{l}
y \\
x \\
\tau \\
\theta
\end{array}\right]+\left[\begin{array}{l}
0 \\
z \\
\kappa \\
0
\end{array}\right]=\left[\begin{array}{l}
0 \\
0 \\
0 \\
\bar{\alpha}
\end{array}\right] } \\
& x \in K, z \in K^{*}, \tau, \kappa \geq 0, y \in \mathbb{R}^{m}, \theta \in \mathbb{R},
\end{aligned}
$$

where for a given $\left(x_{0}, y_{0}, z_{0}, \tau_{0}, \kappa_{0}, \theta_{0}\right) \operatorname{such}$ that $x_{0} \in \operatorname{int}(K), z_{0} \in \operatorname{int}\left(K^{*}\right), \tau_{0}, \kappa_{0}, \theta_{0}>$ 0 ,

$$
\begin{gathered}
\bar{b}=\frac{1}{\theta_{0}}\left(b \tau_{0}-A x_{0}\right), \quad \bar{c}=\frac{1}{\theta_{0}}\left(c \tau_{0}-A^{T} y_{0}-z_{0}\right), \\
\bar{g}=\frac{1}{\theta_{0}}\left(\left\langle c, x_{0}\right\rangle-b^{T} y_{0}+\kappa_{0}\right), \quad \bar{\alpha}=\frac{1}{\theta_{0}}\left(\left\langle x_{0}, z_{0}\right\rangle+\tau_{0} \kappa_{0}\right) .
\end{gathered}
$$

For the (self-dual) model $\left(P_{H}\right)$, we have implemented an algorithm that is analogous to Algorithm IPC in the main program HSDsqlp.m. At each iteration of the algorithm, 
the computation of the search direction is very similar to the case for the problems $(P)$ and $(D)$. For the benefit of readers who are interested in the implementation, here we shall outline the computation of the HKM-like search direction for $\left(P_{H}\right)$. (It is only HKM-like because, to keep corresponding iterates in the problem and its dual, it is necessary to mix the HKM and dual HKM directions suitably.) Let

$$
\widehat{\mathcal{A}}=\left[\mathcal{A} ;-c^{T} ; \bar{c}^{T}\right], \quad \widehat{y}=[y ; \tau ; \theta] .
$$

The HKM-like search direction for $\left(P_{H}\right)$ is the solution of the following linear system of equations:

$$
\begin{aligned}
\widehat{\mathcal{A}} \Delta x+\widehat{B} \Delta \widehat{y}-[0 ; \Delta \kappa ; 0] & =\widehat{R}_{p}:=[0 ; \kappa ;-\bar{\alpha}]-\widehat{\mathcal{A}} x-\widehat{B} \widehat{y} \\
\widehat{\mathcal{A}}^{T} \Delta \widehat{y}+\Delta z & =R_{d}:=-\widehat{\mathcal{A}}^{T} \widehat{y}-z \\
\Delta x+H(\Delta z) & =R_{c} \\
\Delta \kappa+(\kappa / \tau) \Delta \tau & =R_{t}:=\widehat{\mu} / \tau-\kappa,
\end{aligned}
$$

where $\widehat{\mu}=(\langle x, z\rangle+\tau \kappa) /(n+1)$. In $(27)$, the operator $H$ is understood to be acting on the individual blocks of $\Delta z$ as in $(11)$ and $R_{c}=\left[R_{c}^{s} ; R_{c}^{q} ; R_{c}^{l}\right]$, and

$$
\widehat{B}=\left[\begin{array}{ccc}
0 & -b & \bar{b} \\
b^{T} & 0 & \bar{g} \\
-\bar{b}^{T} & -\bar{g} & 0
\end{array}\right]
$$

From here, the equation analogous to the Schur complement equation in (13) without $A^{u}$ is given as follows:

$$
\left(\widehat{\mathcal{A}} H \widehat{\mathcal{A}}^{T}+\widehat{B}+\operatorname{diag}([0 ; \kappa / \tau ; 0])\right) \Delta \widehat{y}=\widehat{h}:=\widehat{R}_{p}+\widehat{\mathcal{A}}\left(H\left(R_{d}\right)-R_{c}\right)+\left[0 ; R_{t} ; 0\right] .
$$

The main difference between (28) and (13) without $A^{u}$ lies in the term $\widehat{B}$, which is an skew-symmetric matrix with rank at most four, generally containing dense columns. To efficiently solve (28), we use the dense-column handling technique described in Section 5.7 to solve the linear system.

We have found that the solution obtained by the solver HSDsqlp.m based on the HSD model $\left(P_{H}\right)$ is generally more accurate than the one obtained by sqlp.m based on $(P)$ and $(D)$ when the SQLP problem is feasible but either the primal or dual feasible region has an empty interior. In Figure 1, we plotted the accuracies attained by HSDsqlp.m against those attained by sqlp.m for about 200 SQLPs whose primal or dual feasible regions are very likely to have empty interiors (based on the fact that the value $\max \left\{g_{P}, g_{D}\right\}$ described in Section 7 are larger than $\left.10^{12}\right)$. We may observe from the scattered plot in Figure 1 that HSDsqlp.m tends to give more accurate solutions compared to sqlp.m. 


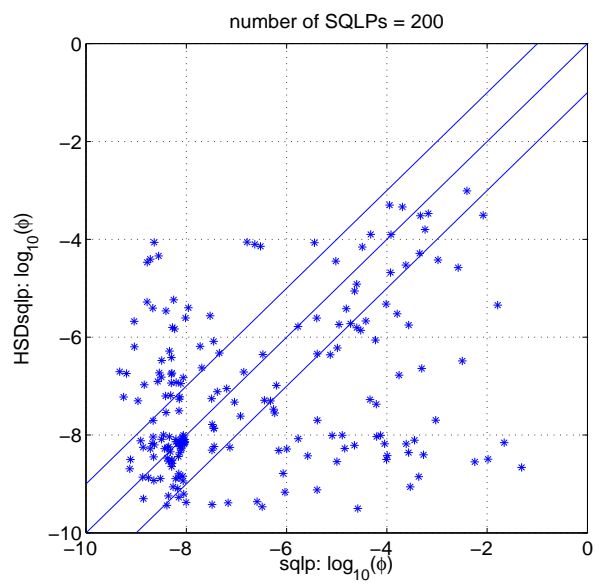

Figure 1: Accuracy attained by HSDsqlp.m versus that attained by sqlp.m for SQLPs whose primal or dual feasible regions have empty interiors.

\section{Detecting feasible regions with empty inte- riors}

In this section, we only consider the case where there are no unrestricted variables $x^{u}$ in $(P)$. Let $d=(A, b, c)$ be the SQLP data associated with $(P)$ and $(D)$ and $F_{P}(d)$ and $F_{D}(d)$ be their respective feasible regions. It is often of interest to know whether the interiors, $F_{P}^{\circ}(d)$ and $F_{D}^{\circ}(d)$, are empty, and how "thick" these regions are. A natural quantitative measure of the "thickness" of $F_{P}(d)$ and $F_{D}(d)$ is the concept of primal and dual distances to infeasibility defined by Renegar [36]:

$$
\rho_{P}(d)=\inf \left\{\|\Delta d\| \|: F_{P}(d+\Delta d)=\emptyset\right\}, \quad \rho_{D}(d)=\inf \left\{\|\Delta d\|: F_{D}(d+\Delta d)=\emptyset\right\} .
$$

With an appropriately chosen norm in the above definitions, the computation of $\rho_{D}(d)$ amounts to solving an SQLP problem with roughly the same dimension and structure as the original primal instance. Unfortunately, the computation of $\rho_{P}(d)$ is extremely costly, requiring the solutions of $2 m$ SQLP problems each with roughly the same dimension and structure as the original dual instance; see [10].

However, in practice, one is typically only interested in the magnitudes of $\rho_{P}(d)$ and $\rho_{D}(d)$ rather than the exact values. It turns out that the following geometric measures proposed by Freund [9] usually give enough information about the magnitudes of $\rho_{P}(d)$ and $\rho_{D}(d)$ :

$$
\begin{aligned}
& g_{P}(d)=\inf \left\{\operatorname { m a x } \left\{\left\|\left|\|x \mid\|, \frac{\|x \mid\|}{r}, \frac{1}{r}\right\}: \mathcal{A}(x)=b, \quad x-r e \in K\right\}\right.\right. \\
& g_{D}(d)=\inf \left\{\max \left\{\|z \mid\|, \frac{\|z\| \mid}{r}, \frac{1}{r}\right\}: \mathcal{A}^{T}(y)+z=c, z-r e \in K\right\},
\end{aligned}
$$

where $e$ is the unit element in $K$ and $|\|\cdot \mid\|$ is an appropriately chosen norm. Note that $g_{P}(d)$ is smaller to the extent that there is a primal feasible solution that is not 
too large and that is not too close to the boundary of $K$. Similar interpretation holds also for $g_{D}(d)$. It can be shown that $g_{P}(d)=\infty \Leftrightarrow \rho_{P}(d)=0$ and $g_{D}(d)=\infty \Leftrightarrow$ $\rho_{D}(d)=0$.

Freund [9] showed that $g_{P}(d)\left(g_{D}(d)\right)$ can be computed at the cost of solving a single SQLP problem with roughly the same dimension and structure as the original primal (dual) instance. In the current release of SDPT3, we include the following m-files to compute $g_{P}(d)$ and $g_{D}(d)$ :

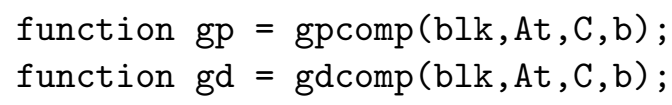

The above routines are based on the standard SQLP formulations of (29) and (30) derived in [10].

Let $\left(x^{*}, y^{*}, z^{*}\right)$ be an optimal solution to $(P)$ and $(D)$, respectively. The geometric measures $g_{P}, g_{D}$ and $\max \left\{\left\|x^{*}\right\|,\left\|z^{*}\right\|\right\}$ for the test problems considered in Section 8 are listed in Table 1 . In the table, we declare that $g_{P}\left(g_{D}\right)$ is equal to $\infty$ if the computed number is larger than $10^{12}$. We have observed that there is strong correlation between $g:=\max \left\{g_{P}, g_{D}\right\} \max \left\{\left\|x^{*}\right\|,\left\|z^{*}\right\|\right\}$ and the accuracy $\phi$ one can obtain when solving $(P)$ and $(D)$. In Figure 2, we plotted the measure $g$ and the accuracy $\phi$ attainable by sqlp.m and HSDsqlp.m for about 420 SQLP problems. The sample correlation between $\log _{10}(\phi)$ with $\phi$ obtained by sqlp.m and $\log _{10}(g)$ for about 170 SQLPs with finite $g$ has a correlation coefficient of 0.75 . Coincidentally, we have the same correlation coefficient for the case where $\phi$ is obtained by HSDsqlp.m.
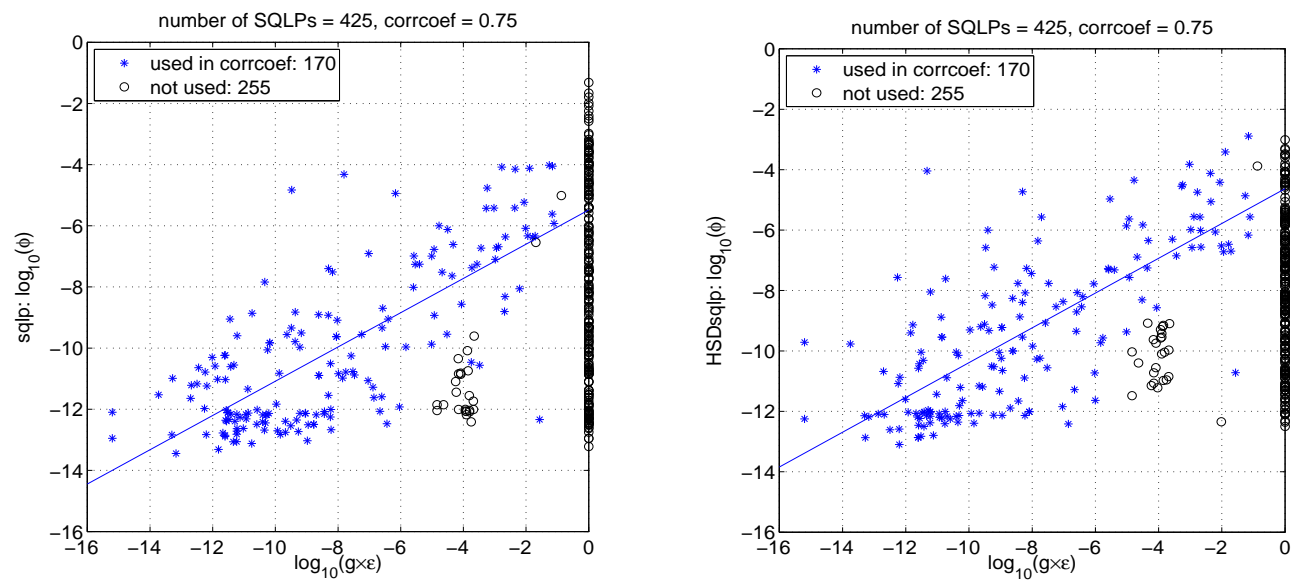

Figure 2: The data points corresponding to "o" are not used in the calculation of the correlation coefficient because either $1 / \max \left\{g_{P}, g_{D}\right\}=0$ or because it is not computed to sufficient accuracy to determine whether the number is in fact 0 . On the $x$-axis, $\varepsilon=$ $2.2 \times 10^{-16}$, and $g=\max \left\{g_{P}, g_{D}\right\} \max \left\{\left\|x^{*}\right\|,\left\|z^{*}\right\|\right\}$. 


\section{Computational results}

Here we describe the results of our computational testing of SDPT3-4.0 using the default parameters, on problems from the following sources:

1. SDPLIB collection of Borchers, available at http://www.nmt.edu/ borchers/sdplib.html

2. DIMACS Challenge test problems, available at http://dimacs.rutgers.edu/Challenges/Seventh/Instances/

3. Sparse SDPs from structural optimization, available at http://www2.am. uni-erlangen.de/ kocvara/pennon/problems.html

4. Sparse SDP collection of Hans Mittelmann, available at ftp://plato.asu.edu/pub/sdp/

5. SDPs from electronic structure calculations, available at http://www.cims.nyu.edu/ mituhiro/software.html

6. SDPs from polynomial optimizations [17].

7. SOCP problems generated by the MATLAB FIR filter toolbox, available at http://www.csee. umbc.edu/ dschol2/opt.html

8. SDPs from polynomial optimizations [37].

Our results were obtained on an Intel Xeon $3.0 \mathrm{GHz}$ PC with $4 \mathrm{G}$ of memory running Linux and MATLAB 7.6. Figure 3 shows the performance of the hybrid solver sdpt3.m on a total of about 430 SQLP problems. It shows that sdpt3.m was able to solve more than $80 \%$ of the problems to an accuracy of at least $10^{-6}$ in the measure $\phi$ defined in (6).

Detailed information such as primal and dual objective values, error measures such as pinfeas, dinfeas, relgap as defined in (6), the geometric measures $g_{P}, g_{D}$, $\max \left\{\left\|x^{*}\right\|,\left\|z^{*}\right\|\right\}$, and the CPU time taken for each problem can be found in Table 1.

\section{$9 \quad$ Future work}

In a future release of SDPT3, we plan to include an implementation of a path-following algorithm which targets high accuracy solutions at the expense of significantly longer computing time per iteration. The key idea would be to compute the search direction at each iteration based on a reduced augmented equation as formulated in [35] and [4] that has at most twice the dimension of the Schur complement equation.

\section{Appendix: A primal-dual infeasible-interior-point algorithm}

Here we give a pseudo-code for the algorithm we implemented. Note that this description makes references to earlier sections where details related to the algorithm are explained. 


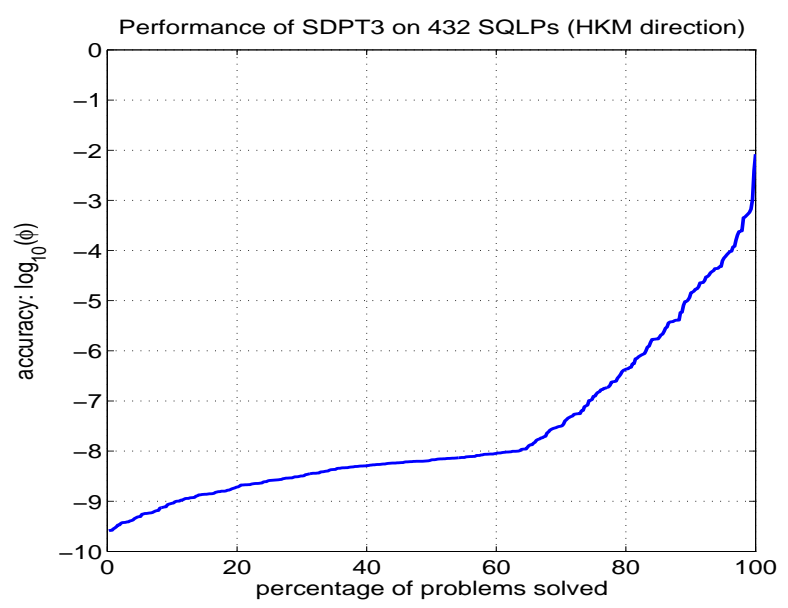

Figure 3: $\phi$ is defined as in (6).

Algorithm IPC. Suppose we are given an initial iterate $\left(x^{0}, y^{0}, z^{0}\right)$ with $x^{0}, z^{0}$ strictly satisfying all the conic constraints. Decide on the type of search direction to use. Set $\bar{\gamma}^{0}=0.9$.

For $k=0,1, \ldots$

(Let the current and the next iterate be $(x, y, z)$ and $\left(x^{+}, y^{+}, z^{+}\right)$respectively. Also, let the current and the next step-length parameter be denoted by $\bar{\gamma}$ and $\bar{\gamma}^{+}$respectively.)

- Compute $\mu(x, z)$ defined in (5), and the accuracy measure $\phi$ defined in (6). Stop the iteration if $\phi$ is sufficiently small.

- (Predictor step) Solve the linear system (13) with $\sigma=0$ in the right-side vector (15). Denote the solution of (11) by $(\delta x, \delta y, \delta z)$. Let $\alpha_{p}$ and $\beta_{p}$ be the steplengths defined as in (23) and (24) with $\Delta x, \Delta z$ replaced by $\delta x, \delta z$, respectively.

- Take $\sigma$ to be

$$
\sigma=\min \left(1,\left[\frac{\mu\left(x+\alpha_{p} \delta x, z+\beta_{p} \delta z\right)}{\mu(x, z)}\right]^{e}\right)
$$

where the exponent $e$ is chosen as follows:

$$
e= \begin{cases}\max \left[1,3 \min \left(\alpha_{p}, \beta_{p}\right)^{2}\right] & \text { if } \mu(x, z)>10^{-6} \\ 1 & \text { if } \mu(x, z) \leq 10^{-6}\end{cases}
$$

- (Corrector step) Solve the linear system (13) with $R_{c}$ in the the right-hand side vector (15) replaced by

$\widehat{R}_{c}^{\tau}=R_{c}^{\tau}-$ Mehrotra-corrector term generated from $\delta x^{\tau}$ and $\delta z^{\tau}, \quad \tau \in\{s, q, l\}$.

Denote the solution of (11) by $(\Delta x, \Delta y, \Delta z)$. 
- Update $(x, y, z)$ to $\left(x^{+}, y^{+}, z^{+}\right)$by

$$
x^{+}=x+\alpha \Delta x, \quad y^{+}=y+\beta \Delta y, \quad z^{+}=z+\beta \Delta z,
$$

where $\alpha$ and $\beta$ are computed as in (23) and (24) with $\bar{\gamma}$ chosen to be $\bar{\gamma}=$ $0.9+0.09 \min \left(\alpha_{p}, \beta_{p}\right)$.

- Update the step-length parameter by $\bar{\gamma}^{+}=0.9+0.09 \min (\alpha, \beta)$.

\section{References}

[1] F. Alizadeh, J.-P. A. Haeberly, and M. L. Overton, Primal-dual interior-point methods for semidefinite programming: convergence results, stability and numerical results, SIAM J. Optimization, 8 (1998), pp. 746-768.

[2] B. Borchers, SDPLIB 1.2, a library of semidefinite programming test problems, Optimization Methods and Software, 11 \& 12 (1999), pp. 683-690. Available at http://www.nmt.edu/ ${ }^{\sim}$ borchers/sdplib.html.

[3] S. Boyd, L. El Ghaoui, E. Feron, and V. Balakrishnan. Linear Matrix Inequalities in System and Control Theory. SIAM Studies in Applied Mathematics. SIAM, Philadelphia, USA, 1994

[4] Z. Cai and K.C. Toh, Solving second order cone programming via the augmented systems, SIAM J. Optimization, 17 (2006), pp. 711-737.

[5] T. A. Davis, CHOLMOD Version 1.0 User Guide, Dept. of Computer and Information Science and Engineering, Univ. of Florida, Gainesville, FL, 2005. Details available at http://www.cise.ufl.edu/research/sparse/cholmod

[6] T. A. Davis, UMFPACK Version 4.6 User Guide, Dept. of Computer and Information Science and Engineering, Univ. of Florida, Gainesville, FL, 2002. Details available at http://www.cise.ufl.edu/research/sparse/umfpack

[7] I. S. Duff, MA57 A new code for the solution of sparse symmetric definite and indefinite systems, Technical Report RAL-TR-2002-024, Rutherford Appleton Laboratory, 2002.

[8] M. Grant and S. Boyd, Graph implementations for nonsmooth convex programs, in Recent Advances in Learning and Control (a tribute to M. Vidyasagar), V. Blondel, S. Boyd, and H. Kimura, editors, Lecture Notes in Control and Information Sciences, Springer, 2008, pp. 95-110. Software is available at http://cvxr.com/cvx/

[9] R. M. Freund, Complexity of convex optimization using geometry-based measures and a reference point, Mathematical Programming, 99 (2004), pp. 197-221.

[10] R.M. Freund, F. Ordóñez, and K.-C. Toh, Behavioral Measures and their Correlation with IPM Iteration Counts on Semi-Definite Programming Problems, Mathematical Programming, 109 (2007), pp. 445-475. 
[11] R. W. Freund and N. M. Nachtigal, A new Krylov-subspace method for symmetric indefinite linear systems, Proceedings of the 14th IMACS World Congress on Computational and Applied Mathematics, Atlanta, USA, W.F. Ames ed., July 1994, pp. 1253-1256.

[12] K. Fujisawa, M. Kojima, K. Nakata, and M. Yamashita, SDPA (SemiDefinite Programming Algorithm) User's manual - version 6.2.0, Research Report B308, Department Mathematical and Computing Sciences, Tokyo Institute of Technology, December 1995, revised September 2004.

[13] K. Fujisawa, M. Kojima, and K. Nakata, Exploiting sparsity in primal-dual interior-point method for semidefinite programming, Mathematical Programming, 79 (1997), pp. 235-253.

[14] G. H. Golub and C. F. Van Loan, Matrix Computations, 2nd ed., Johns Hopkins University Press, Baltimore, MD, 1989.

[15] Harwell Subroutine Library: http://www.cse.clrc.ac.uk/Activity/HSL

[16] C. Helmberg, F. Rendl, R. Vanderbei, and H. Wolkowicz, An interior-point method for semidefinite programming, SIAM Journal on Optimization, 6 (1996), pp. 342-361.

[17] D. Henrion, private communication.

[18] N. J. Higham, Computing the nearest correlation matrix - a problem from finance, IMA J. Numerical Analysis, 22 (2002), pp. 329-343.

[19] N. J. Higham, Accuracy and Stability of Numerical Algorithms, SIAM, Philadelphia, 1996.

[20] M. Kojima, S. Shindoh, and S. Hara, Interior-point methods for the monotone linear complementarity problem in symmetric matrices, SIAM J. Optimization, 7 (1997), pp. 86-125.

[21] J. Löfberg, A Toolbox for Modeling and Optimization in MATLAB, Proceedings of the CACSD Conference, 2004, Taipei, Taiwan.

Available at http://control.ee.ethz.ch/ joloef/yalmip.php

[22] M. S. Lobo, L. Vandenberghe, S. Boyd and H. Lebret, Applications of Secondorder Cone Programming, Linear Algebra Appl., 284 (1998), pp. 193-228.

[23] S. Mehrotra, On the implementation of a primal-dual interior point method, SIAM J. Optimization, 2 (1992), pp. 575-601.

[24] R. D. C. Monteiro, Primal-dual path-following algorithms for semidefinite programming, SIAM J. Optimization, 7 (1997), pp. 663-678.

[25] R. D. C. Monteiro, and T. Tsuchiya, Polynomial convergence of primal-dual algorithms for the second-order cone program based on the MZ-family of directions, Mathematical Programming, 88 (2000), pp. 61-83.

[26] J.W. Liu, E.G. Ng, and B.W. Peyton, On finding supernodes for sparse matrix computations, SIAM J. Matrix Anal. Appl., 1 (1993), pp. 242-252. 
[27] G. Pataki and S. Schmieta, The DIMACS library of mixed semidefinite-quadraticlinear programs.

Available at http://dimacs.rutgers.edu/Challenges/Seventh/Instances

[28] Yu. E. Nesterov and M. J. Todd, Self-scaled barriers and interior-point methods in convex programming, Math. Oper. Res., 22 (1997), pp. 1-42.

[29] J. F. Sturm, Using SeDuMi 1.02, a Matlab toolbox for optimization over symmetric cones, Optimization Methods and Software, 11 \& 12 (1999), pp. 625-653.

[30] M. J. Todd, K. C. Toh, and R. H. Tütüncü, On the Nesterov-Todd direction in semidefinite programming, SIAM J. Optimization, 8 (1998), pp. 769-796.

[31] K. C. Toh, A note on the calculation of step-lengths in interior-point methods for semidefinite programming, Computational Optimization and Applications, 21 (2002), pp. 301-310.

[32] K. C. Toh, Some new search directions for primal-dual interior point methods in semidefinite programming, SIAM J. Optimization, 11 (2000), pp. 223-242.

[33] K. C. Toh, Primal-dual path-following algorithms for determinant maximization problems with linear matrix inequalities, Computational Optimization and Applications, 14 (1999), pp. 309-330.

[34] K. C. Toh, M. J. Todd, and R. H. Tütüncü, SDPT3 - a Matlab software package for semidefinite programming, Optimization Methods and Software, 11/12 (1999), pp. 545-581.

[35] K. C. Toh, Solving large scale semidefinite programs via an iterative solver on the augmented systems, SIAM J. Optim., 14 (2003), pp. 670-698.

[36] J. Renegar, Linear programming, complexity theory, and elementary functional analysis, Mathematical Programming, 70 (1995), pp. 279-351.

[37] H. Waki, S. Kim, M. Kojima, M. Muramatsu and H. Sugimoto, SparsePOP: a Sparse Semidefinite Programming Relaxation of Polynomial Optimization Problems, ACM Transactions on Mathematical Software, 35 (2008), article 15.

[38] T. Tsuchiya, A convergence analysis of the scaling-invariant primal-dual pathfollowing algorithms for second-order cone programming, Optimization Methods and Software, 11/12 (1999), pp. 141-182.

[39] R. H. Tütüncü, K. C. Toh, and M. J. Todd, Solving semidefinite-quadratic-linear programs using SDPT3, Mathematical Programming Ser. B, 95 (2003), pp. 189217.

[40] L. Vandenberghe, S. Boyd, and S.-P. Wu, Determinant maximization with linear matrix inequalities, SIAM J. Matrix Analysis and Applications, 19 (1998), pp. 499-533.

[41] Y. Ye, M.J. Todd, and S. Mizuno, An $O(\sqrt{n} L)$-iteration homogeneous and selfdual linear programming algorithm, Mathematics of Operations Research, 19 (1994), pp. 53-67. 
Table 1: Performance of sdpt3.m. In the table, err = [pinfeas, dinfeas, relgap, relgap2], where relgap2 is the same as relgap but with the numerator replaced by $\left|\langle c, x\rangle-b^{T} y\right|$, and normXZ $=\max \left\{\left\|x^{*}\right\|,\left\|z^{*}\right\|\right\}$. We declare that $g_{P}\left(g_{D}\right)$ is $\infty$ if the computed number is larger than $10^{12}$

\begin{tabular}{|c|c|c|c|c|c|}
\hline problem & $m \mid n_{s} ; n_{q} ; n_{l} ; n_{u}$ & it. | primal obj | dual obj & err & time & $g_{P}\left|g_{D}\right|$ normXz \\
\hline $\operatorname{arch} 0$ & $174 \mid 161 ; ; 174$ & \begin{tabular}{l|l|l}
26 & $-5.66517270-1 \mid$ & $-5.66517274-1$
\end{tabular} & $\begin{array}{l}5.4-9|1.6-11| \\
\end{array} 1.9-9 \mid 1.8-9$ & 03 & $\begin{array}{ll}1.974 \mid & 2.036 \mid 5.92\end{array}$ \\
\hline $\operatorname{arch} 2$ & $174 \mid 161 ; ; 174$ & $24|-6.71515400-1|-6.71515409-1$ & $4.2-10|3.0-11| 4.0-9 \mid 3.7-9$ & 03 & $1.974|2.016| 5.72$ \\
\hline $\operatorname{arch} 4$ & $174 \mid 161 ; ; 174$ & $22|-9.72627409-1|-9.72627419-1$ & $7.3-10|1.9-11| 3.8-9 \mid 3.7-9$ & 03 & $1.974|1.966| 9.12$ \\
\hline $\operatorname{arch} 8$ & $174 \mid 161 ; ; 174$ & $23|-7.056980020|-7.056980040$ & $9.7-9|2.9-11| 1.5-9 \mid 1.2-9$ & 03 & $1.974|1.836| 6.33$ \\
\hline control1 & $21 \mid 15 ; ; ;$ & $17|-1.778462681|-1.778462671$ & $3.1-9|2.7-11| 1.8-9 \mid 3.2-10$ & 00 & $9.314|4.983| 5.75$ \\
\hline control2 & $66 \mid 30 ; ; ;$ & $21|-8.300000390|-8.299999990$ & $7.1-9|3.0-11| 9.4-11 \mid 2.3-8$ & 01 & $3.035|1.474| 6.55$ \\
\hline control3 & $136 \mid 45 ;$; ; & $22|-1.363326471|-1.363326721$ & $1.9-7|8.7-11| 1.0-7 \mid 8.9-8$ & 02 & \begin{tabular}{ll|l|l|l|l}
7.67 & 5.16 & 4 & 2.16
\end{tabular} \\
\hline control4 & $231 \mid 60 ; ;$ & $21|-1.979423251|-1.979423081$ & $1.9-7|1.5-10| 1.7-8 \mid 4.1-8$ & 04 & $1.346|4.924| 3.86$ \\
\hline control5 & $351 \mid 75 ; ;$ & $23|-1.688359361|-1.688360101$ & $4.3-7|1.6-10| 2.5-7 \mid 2.1-7$ & 11 & $2.026|6.224| 4.96$ \\
\hline control6 & $496 \mid 90 ; ;$ & $21|-3.730436481|-3.730442731$ & $2.2-7|5.8-10| 8.1-7 \mid 8.3-7$ & 22 & $3.126|9.214| 1.47$ \\
\hline control7 & $666 \mid 105 ;$; ; & $22|-2.062505811|-2.062507781$ & $5.9-8|4.9-10| 4.7-7 \mid 4.7-7$ & 43 & 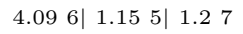 \\
\hline control8 & $861 \mid 120 ; ;$ & $23|-2.028634781|-2.028636531$ & $2.0-7|4.8-10| 4.0-7 \mid 4.2-7$ & $1: 23$ & $\begin{array}{lllll}5.53 & 6 \mid & 1.40 & 5 & 1.37\end{array}$ \\
\hline control9 & $1081 \mid 135 ;$; ; & $23|-1.467541571|-1.467542841$ & $2.7-7|4.7-10| 4.6-7 \mid 4.2-7$ & $2: 24$ & 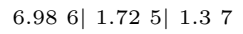 \\
\hline control10 & $1326 \mid 150 ; ; ;$ & $25|-3.853286871|-3.853305821$ & $5.0-7 \mid$\begin{tabular}{ll|ll|ll}
2 & & 2.4 & -9 & -6 & -6
\end{tabular} & 45 & $8.326|2.005| 3.77$ \\
\hline control11 & $1596 \mid 165 ; ; ;$ & $24|-3.195860901|-3.195868621$ & $5.9-7 \mid$\begin{tabular}{ll|ll|ll} 
& 1.3 & -9 & 8.7 & -7 & -6
\end{tabular} & $1: 07$ & $1.027|2.315| 3.47$ \\
\hline gpp100 & $101 \mid 100 ; ;$ & $14|4.494354791| 4.494354891$ & $2.7-10|6.2-11| 9.8-9 \mid 1.1-8$ & 00 & | $1.882 \mid 6.24$ \\
\hline gpp124-1 & $125 \mid 124 ; ; ;$ & $17|7.343075250| 7.343075710$ & $3.6-11|7.1-12| 7.6-9 \mid 2.9-8$ & 01 & | $1.922 \mid 1.75$ \\
\hline gpp124-2 & $125 \mid 124 ; ; ;$ & $15|4.686229331| 4.686229391$ & $1.1-10|2.2-11| 6.1-9 \mid 6.3-9$ & 01 & | $2.372 \mid 1.15$ \\
\hline gpp124-3 & $125 \mid 124 ; ; ;$ & $14 \mid 1.530141232$ | 1.530141242 & $4.2-10|8.5-11| 4.1-9 \mid 5.1-9$ & 01 & | $2.832 \mid 6.94$ \\
\hline gpp124-4 & $125 \mid 124 ; ;$; & $15|4.189875952| 4.189876102$ & $5.6-10|7.1-11| 6.3-10 \mid 1.8-8$ & 01 & | $3.482 \mid 2.95$ \\
\hline gpp250-1 & $251 \mid 250 ; ;$ & $18|1.544491681| 1.544491681$ & $1.3-12|1.5-12| 2.9-9 \mid 8.7-11$ & 02 & | $4.012 \mid 1.06$ \\
\hline gpp250-2 & $251 \mid 250 ; ;$ & $15|8.186895621| 8.186895741$ & $1.3-10|2.6-11| 1.2-9 \mid 7.4-9$ & 02 & | $4.762 \mid 1.65$ \\
\hline gpp250-3 & $251 \mid 250 ; ;$ & $15|3.035393172| 3.035393202$ & $3.3-10|6.7-11| 2.1-9 \mid 5.2-9$ & 02 & | $5.912 \mid 1.95$ \\
\hline gpp250-4 & $251 \mid 250 ; ;$ & $14|7.473283062| 7.473283052$ & $2.2-10|5.3-11| 4.6-9 \mid 5.8-10$ & 02 & $\mid \begin{array}{ll}7.202 \mid & 4.05\end{array}$ \\
\hline gpp500-1 & $501 \mid 500 ; ;$ & $20|2.532055081| 2.532054361$ & $1.6-12|2.6-12| 1.4-7 \mid 1.4-7$ & 12 & | $7.882 \mid 1.37$ \\
\hline gpp500-2 & $501 \mid 500 ; ; ;$ & $19|1.560603872| 1.560603872$ & $3.1-12|6.9-12| 6.4-10 \mid 7.7-11$ & 11 & | $9.572 \mid 1.66$ \\
\hline gpp500-3 & $501 \mid 500 ; ;$ & $16|5.130176102| 5.130176022$ & $4.3-12|2.0-12| 7.7-9 \mid 7.5-9$ & 10 & | $1.173 \mid 1.06$ \\
\hline gpp500-4 & $501 \mid 500 ; ;$ & $17|1.567018793| 1.567018793$ & $8.8-12|3.8-12| 9.2-10 \mid 8.2-10$ & 10 & | $1.503 \mid 7.75$ \\
\hline hinf1 & $13 \mid 14 ; ; ;$ & $23|-2.032726560|-2.032674450$ & $1.3-7|4.1-8| 3.4-6 \mid 1.0-5$ & 00 & | $7.621 \mid 9.23$ \\
\hline hinf2 & $13 \mid 16 ; ; ;$ & $16|-1.096925351|-1.096815261$ & $3.3-6|1.5-11| 2.6-9 \mid 4.8-5$ & 00 & $1.515|5.053| 4.62$ \\
\hline hinf3 & $13 \mid 16 ; ; ;$ & $21|-5.696791341|-5.695434381$ & $7.1-6|8.1-12| 3.1-9 \mid 1.2-4$ & 00 & | $1.484 \mid 4.43$ \\
\hline $\operatorname{hinf} 4$ & $13 \mid 16 ; ; ;$ & $21|-2.747657222|-2.747647912$ & 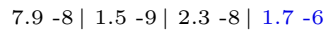 & 00 & | $1.763 \mid 4.64$ \\
\hline hinf5 & $13 \mid 16 ; ; ;$ & $21|-3.628973522|-3.625571022$ & 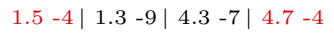 & 00 & | $1.045 \mid 2.04$ \\
\hline hinf6 & $13 \mid 16 ; ; ;$ & $22|-4.489727382|-4.489523532$ & $1.5-5|1.3-8| 3.6-6 \mid 2.3-5$ & 00 & | $6.764 \mid 1.25$ \\
\hline $\operatorname{hinf7}$ & $13 \mid 16 ; ; ;$ & $18|-3.908266762|-3.908199182$ & $9.6-6|1.8-10| 1.1-6 \mid 8.6-6$ & 00 & | $3.555 \mid 2.74$ \\
\hline hinf8 & $13 \mid 16 ; ; ;$ & $21|-1.161910712|-1.161685102$ & $2.8-5|1.0-11| 3.3-9 \mid 9.7-5$ & 00 & | $1.634 \mid 2.54$ \\
\hline hinf9 & $13 \mid 16 ; ; ;$ & $21|-2.362492772|-2.362492582$ & $1.0-6|1.4-14| 4.1-10 \mid 3.9-8$ & 00 & $3.092|1.016| 7.54$ \\
\hline hinf10 & $21 \mid 18 ; ; ;$ & $28|-1.088336662|-1.087815522$ & $1.3-7|6.8-8| 6.0-5 \mid 2.4-4$ & 00 & | $1.603 \mid 1.76$ \\
\hline hinf11 & $31 \mid 22 ; ; ;$ & $25|-6.593499481|-6.591690261$ & \begin{tabular}{ll|ll|ll|ll}
3.7 & -7 & 1.7 & -7 & 4.5 & -4 & 1.4 & -4
\end{tabular} & 01 & | $1.263 \mid 1.16$ \\
\hline hinf12 & $43 \mid 24 ; ; ;$ & $60|-6.92650138-5|-5.39089136-5$ & $8.9-12|3.0-6| \begin{array}{lllll}4 & 4.8 & -5 & 1.5 & -5\end{array}$ & 01 & | $1.423 \mid 4.111$ \\
\hline hinf13 & $57 \mid 30 ; ; ;$ & $32|-4.435396041|-4.434956521$ & $5.1-5|3.1-7| 2.0-4 \mid 4.9-5$ & 01 & $|9.374| 1.87$ \\
\hline hinf14 & $73 \mid 34 ; ; ;$ & $29|-1.299007521|-1.299006681$ & 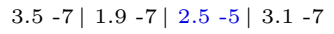 & 01 & | $3.283 \mid 8.05$ \\
\hline hinf15 & $91 \mid 37 ; ; ;$ & $30|-2.401079401|-2.400660891$ & 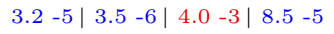 & 01 & $|1.785| 1.26$ \\
\hline $\operatorname{infd} 1$ & $10 \mid 30 ; ; ;$ & $11|-4.257208010| 1.5422863919$ & primal infeasible & 00 & \\
\hline $\operatorname{infd} 2$ & $10 \mid 30 ; ; ;$ & $11|5.260014440| 2.0993105820$ & primal infeasible & 00 & \\
\hline $\operatorname{infp} 1$ & $10 \mid 30 ; ; ;$ & $31|-9.4170967815|-9.565045090$ & dual infeasible & 00 & \\
\hline $\operatorname{infp} 2$ & $10 \mid 30 ; ; ;$ & $31|-3.0046017715|-7.565879830$ & dual infeasible & 00 & \\
\hline $\operatorname{mcp} 100$ & $100 \mid 100 ; ; ;$ & $12|-2.261573512|-2.261573522$ & $1.2-11|1.0-12| 2.0-9 \mid 2.0-9$ & 00 & $1.002|1.922| 5.01$ \\
\hline mcp124-1 & $124 \mid 124 ; ; ;$ & $12|-1.419904752|-1.419904772$ & $3.6-12|1.0-12| 7.5-9 \mid 7.5-9$ & 00 & $1.242|1.912| 6.51$ \\
\hline mcp124-2 & $124 \mid 124 ; ; ;$ & $13|-2.698801702|-2.698801712$ & $2.0-13|1.1-12| 4.1-10 \mid 4.1-10$ & 01 & \begin{tabular}{ll|ll|ll}
1.24 & 2 & 2.35 & 2 & 6.31
\end{tabular} \\
\hline mcp124-3 & $124 \mid 124 ; ; ;$ & $12|-4.677501102|-4.677501142$ & $6.5-13|1.0-12| 4.9-9 \mid 4.9-9$ & 01 & $1.242|2.822| 6.11$ \\
\hline mcp124-4 & $124 \mid 124 ; ; ;$ & $13|-8.644118632|-8.644118642$ & $3.2-12|1.5-12| 4.0-10 \mid 4.0-10$ & 01 & $1.242|3.532| 6.51$ \\
\hline mcp250-1 & $250 \mid 250 ; ;$ & $14|-3.172643402|-3.172643402$ & $6.7-13|1.0-12| 9.7-10 \mid 9.7-10$ & 01 & $\begin{array}{llll}2.50 & 2 & 4.02 & 2 \mid \\
1.32\end{array}$ \\
\hline mcp250-2 & $250 \mid 250 ; ;$ & $13|-5.319300812|-5.319300842$ & $1.4-11|1.0-12| 2.6-9 \mid 2.6-9$ & 01 & $2.502|4.762| 1.12$ \\
\hline $\mathrm{mcp} 250-3$ & $250 \mid 250 ; ; ;$ & $13|-9.811725662|-9.811725722$ & $6.9-12|1.0-12| 2.8-9 \mid 2.8-9$ & 01 & $\begin{array}{lllll}2.50 & 2 & 5.90 & 2 & 1.02\end{array}$ \\
\hline mcp250-4 & $250 \mid 250 ; ;$ & $14|-1.681960103|-1.681960113$ & $1.8-13|1.0-12| 4.5-9 \mid 4.5-9$ & 01 & $2.502|7.212| 1.12$ \\
\hline mcp500-1 & $500 \mid 500 ; ;$ & $15|-5.981485162|-5.981485172$ & $8.5-13|1.0-12| 5.5-10 \mid 5.5-10$ & 04 & $\begin{array}{lllll}5.002 \mid & 7.86 & 2 & 2.32\end{array}$ \\
\hline mcp500-2 & $500 \mid 500 ; ;$ & $16|-1.070056763|-1.070056773$ & $4.1-13|1.2-12| 1.2-9 \mid 1.2-9$ & 05 & $5.002|9.592| 2.12$ \\
\hline $\operatorname{mcp} 500-3$ & $500 \mid 500 ; ; ;$ & $14|-1.847969993|-1.847970023$ & $1.1-12|1.0-12| 9.2-9 \mid 9.2-9$ & 05 & $5.002|1.173| 1.92$ \\
\hline mcp500-4 & $500 \mid 500 ; ; ;$ & $13|-3.566737993|-3.566738053$ & $3.4-12|1.0-12| 8.8-9 \mid 8.8-9$ & 05 & $5.002|1.513| 1.92$ \\
\hline qap5 & $136 \mid 26 ; ;$ & $10|4.360000002| 4.360000002$ & $8.3-12|2.7-10| 7.5-10 \mid 4.4-10$ & 01 & | $1.353 \mid 8.73$ \\
\hline qap6 & $229 \mid 37 ; ; ;$ & \begin{tabular}{l|l|l|l|l|}
18 & 3.813931572 & 3.814161612
\end{tabular} & $4.6-7|1.8-10| 2.6-9 \mid 3.0-5$ & 02 & | $3.333 \mid 5.14$ \\
\hline
\end{tabular}


Table 1: Performance of sdpt3.m. In the table, err = [pinfeas, dinfeas, relgap, relgap2], where relgap2 is the same as relgap but with the numerator replaced by $\left|\langle c, x\rangle-b^{T} y\right|$, and normXZ $=\max \left\{\left\|x^{*}\right\|,\left\|z^{*}\right\|\right\}$. We declare that $g_{P}\left(g_{D}\right)$ is $\infty$ if the computed number is larger than $10^{12}$.

\begin{tabular}{|c|c|c|c|c|c|}
\hline problem & $m \mid n_{s} ; n_{q} ; n_{l} ; n_{u}$ & it. | primal obj | dual obj & err & time & $g_{P}\left|g_{D}\right|$ normXZ \\
\hline qap7 & $358 \mid 50 ; ;$ & \begin{tabular}{l|l|l|l} 
& 4.247881352 & 2 & 4.248041492
\end{tabular} & $3.3-7|3.0-10| 4.2-9 \mid 1.9-5$ & 01 & | $4.073 \mid 5.84$ \\
\hline qap8 & $529 \mid 65 ; ;$ & $17|7.568416472| 7.568992432$ & $9.1-7 \mid \begin{array}{lllllll}2 & 2.5 & -9 & 3.1 & -8 & 3.8 & -5\end{array}$ & 01 & | $7.073 \mid 7.84$ \\
\hline qap9 & $748 \mid 82 ; ; ;$ & \begin{tabular}{l|l|l|l|l|}
17 & 1.409918663 & 3 & 1.409929933
\end{tabular} & \begin{tabular}{lll|ll|ll|ll}
5.8 & -8 & 1.7 & -9 & 1.5 & -8 & 4.0 & -6
\end{tabular} & 02 & $\mid$\begin{tabular}{ll|l}
1.11 & $4 \mid$ & 2.75
\end{tabular} \\
\hline qap10 & $1021 \mid 101 ; ; ;$ & $17|1.092540453| 1.092574363$ & $2.7-7|1.9-9| 2.0-8 \mid 1.6-5$ & 05 & $|1.464| 1.65$ \\
\hline ss 30 & $132 \mid 294 ; ; 132$ & $22|-2.023950961|-2.023951061$ & $1.2-7|5.8-11| 3.2-8 \mid 2.4-8$ & 12 & $1.023|2.405| 1.83$ \\
\hline theta1 & $104 \mid 50 ; ;$ & $11|-2.299999971|-2.300000011$ & $1.1-11|4.6-12| 8.2-9 \mid 8.2-9$ & 00 & $5.001|1.153| 2.12$ \\
\hline theta2 & $498 \mid 100 ; ;$ & $13|-3.287916891|-3.287916901$ & $1.2-12|1.3-12| 1.4-9 \mid 1.4-9$ & 01 & $1.002|3.293| 4.32$ \\
\hline theta3 & $1106 \mid 150 ; ; ;$ & $14|-4.216698131|-4.216698151$ & $3.5-11|1.0-12| 2.6-9 \mid 2.6-9$ & 02 & $1.502|6.333| 6.62$ \\
\hline theta4 & $1949 \mid 200 ;$; ; & $14|-5.032122131| \begin{array}{l}-5.03212220 \\
14\end{array}$ & $2.4-13|1.0-12| 7.0-9 \mid 7.0-9$ & 08 & $2.002|1.014| 9.12$ \\
\hline theta5 & $3028 \mid 250 ; ; ;$ & $14|-5.723230691|-5.723230731$ & $1.8-13|1.0-12| 3.5-9 \mid 3.5-9$ & 24 & $2.502|1.434| 1.23$ \\
\hline theta6 & $4375 \mid 300 ; ; ;$ & $14|-6.347708701|-6.347708721$ & $1.4-12|1.0-12| 1.5-9 \mid 1.6-9$ & 59 & $3.002|1.904| 1.43$ \\
\hline truss 1 & $6 \mid 12 ; ; 1 ;$ & $9|8.999996510| 8.999996290$ & $2.3-9|9.8-11| 6.9-9 \mid 1.2-8$ & 00 & \begin{tabular}{ll|l|ll}
4.56 & $2 \mid$ & 1.30 & 1 & 2.41
\end{tabular} \\
\hline truss2 & $58 \mid 132 ; ; 1$ & $13|1.233803572| 1.233803562$ & $9.3-10|5.7-10| 2.2-9 \mid 3.7-9$ & 00 & $6.534|1.332| 7.12$ \\
\hline truss 3 & $27 \mid 30 ; ; 1$ & $12|9.109996270| 9.109996130$ & $3.8-14|9.9-13| 7.1-9 \mid 7.1-9$ & 00 & $\begin{array}{llll}1.14 & 3 & 3.10 & 1\end{array} \mid 2.41$ \\
\hline truss 4 & $12 \mid 18 ; ; 1$ & $11|9.009996450| 9.009996290$ & $3.8-9|1.1-11| 8.2-11 \mid 8.2-9$ & 00 & 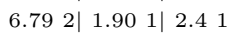 \\
\hline truss5 & $208 \mid 330 ; ; 1$ & \begin{tabular}{l|l|l|l}
15 & 1.32635678 & 2 & 1.326356782
\end{tabular} & $1.5-10|3.3-12| 5.8-10 \mid 5.7-10$ & 01 & $1.755|3.312| 7.62$ \\
\hline truss 6 & $172 \mid 450 ; ; 1$ & $25|9.010014272| 9.010013892$ & $5.5-8|2.3-11| 1.4-8 \mid 2.1-8$ & 01 & $1.626|4.512| 1.14$ \\
\hline truss 7 & $86 \mid 300 ; ; 1 ;$ & $22|9.000015512| 9.000013722$ & $1.3-8|1.7-11| 1.0-7 \mid 9.9-8$ & 00 & 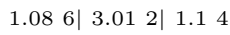 \\
\hline truss 8 & $496 \mid 627 ; ; 1$ & $16|1.331145892| 1.331145892$ & $2.2-10|8.4-12| 2.8-10 \mid 2.8-10$ & 03 & \begin{tabular}{ll|l|ll}
3.35 & 5 & 6.28 & 2 & 7.72
\end{tabular} \\
\hline $\operatorname{maxG11}$ & $800 \mid 800 ; ; ;$ & $15|-6.291647772| \begin{array}{l}-6.291647832 \\
\end{array}$ & $1.3-12|1.0-12| 4.8-9 \mid 4.8-9$ & 12 & $8.002|1.413| 3.82$ \\
\hline $\max G 32$ & $2000 \mid 2000 ; ;$ & $15|-1.567639613|-1.567639643$ & $7.0-12|1.0-12| 9.9-9 \mid 9.9-9$ & $1: 47$ & $2.003|3.563| 7.72$ \\
\hline $\operatorname{maxG51}$ & $1000 \mid 1000 ; ;$ & $17|-4.006255523|-4.006255523$ & $3.6-13|1.0-12| 2.6-10 \mid 2.6-10$ & 28 & $1.003|2.053| 3.22$ \\
\hline qpG11 & $800 \mid 1600 ; ;$ & $15|-2.448659093|-2.448659133$ & $8.6-13|1.0-12| 8.4-9 \mid 8.4-9$ & 12 & $1.603|6.503| 3.82$ \\
\hline qpG51 & $1000 \mid 2000 ; ; ;$ & 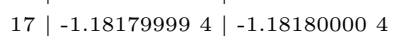 & $1.4-11|1.1-12| 2.1-9 \mid 2.1-9$ & 25 & $2.003|2.564| 9.92$ \\
\hline thetaG11 & $2401 \mid 801 ; ; ;$ & $18|-3.999999952|-4.000000002$ & $5.0-12|1.0-12| 6.3-9 \mid 6.3-9$ & 40 & $2.403|9.472| 8.02$ \\
\hline thetaG51 & $6910 \mid 1001 ; ; ;$ & $39|-3.489999802|-3.490000012$ & $4.0-8|2.3-12| 1.9-8 \mid 2.9-8$ & 18:00 & $3.104|1.103| 7.22$ \\
\hline equalG11 & $801 \mid 801 ; ; ;$ & $17|-6.291552922|-6.291552932$ & $4.6-12|9.9-13| 3.1-10 \mid 2.8-10$ & 31 & $1.603|2.213| 2.65$ \\
\hline equalG51 & $1001 \mid 1001 ; ; ;$ & \begin{tabular}{l|l|l|l}
18 & -4.00560128 & 3 & -4.00560132 \\
\end{tabular} & $5.8-11|5.1-12| 4.3-9 \mid 4.2-9$ & 59 & $2.003|3.053| 4.85$ \\
\hline bm1 & $883 \mid 882 ; ; ;$ & \begin{tabular}{l|l|l|l}
20 & 2.343983451 & 2.343981851
\end{tabular} & $5.9-12|3.1-12| 3.4-7 \mid 3.3-7$ & 44 & | $1.413 \mid 1.27$ \\
\hline copo14 & $1275 \mid 196 ; ; 364$ & $17|3.05271933-10|-8.22282898-10$ & $1.1-13|1.5-12| 1.1-9 \mid 1.1-9$ & 02 & 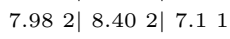 \\
\hline copo 23 & $5820 \mid 529 ; ; 1771$ & $20|2.21718748-10|-5.46605094-10$ & $7.9-13|1.0-12| 7.7-10 \mid 7.7-10$ & 57 & $2.903|3.253| 1.52$ \\
\hline hamming-7- & $1793 \mid 128 ; ;$ & $8|-4.2666666611|-4.266666681$ & $1.0-10|3.7-11| 8.3-9 \mid 8.3-9$ & 03 & $1.282|5.463| 5.02$ \\
\hline hamming-9- & $2305 \mid 512 ; ; ;$ & $9|-2.239999982|-2.240000012$ & $3.4-11|2.2-11| 6.1-9 \mid 6.1-9$ & 10 & \begin{tabular}{ll|l|l}
5.12 & $2 \mid$ & 1.15 & 5 \\
5.53
\end{tabular} \\
\hline minphase & $48 \mid 48 ; ;$ & $11|5.77209594-1| 5.77209579-1$ & $2.6-13|2.3-12| 7.0-9 \mid 6.9-9$ & 00 & $\mid$\begin{tabular}{l|l}
1.18 & $3 \mid$ \\
$\mid$ & 1.92
\end{tabular} \\
\hline torusg3-8 & $512 \mid 512 ; ; ;$ & $15|-4.834094597|-4.834094597$ & $2.5-13|1.0-12| 1.0-9 \mid 1.0-9$ & 05 & $5.122|4.707| 2.66$ \\
\hline toruspm3-8 & $512 \mid 512 ; ; ;$ & $14|-5.278086612| \begin{array}{ll}-5.278086632 \\
\end{array}$ & $4.4-11|1.0-12| 2.2-9 \mid 2.2-9$ & 05 & \begin{tabular}{ll|l|l}
5.12 & $2 \mid$ & $1.043 \mid$ & 2.12
\end{tabular} \\
\hline torusg3-15 & $3375 \mid 3375 ; ;$ & $16|-6.376218453|-6.376218553$ & $3.7-13|1.0-12| 7.4-9 \mid 7.4-9$ & $9: 31$ & $3.383|9.703| 1.13$ \\
\hline toruspm3-1 & $3375 \mid 3375 ; ; ;$ & $16|-3.475131853|-3.475131863$ & $5.7-13|1.0-12| 1.7-9 \mid 1.7-9$ & 9:39 & $3.383|6.853| 9.92$ \\
\hline filter 48 & $969 \mid 48 ; 49 ; 931$ & $41|1.416129150| 1.416128640$ & $9.5-8|1.2-9| 1.7-7 \mid 1.3-7$ & 25 & $1.148|2.303| 4.62$ \\
\hline filtinf1 & $983 \mid 49 ; 49 ; 945$ & $34|0.00000000-16| 1.206098622$ & primal infeasible & 22 & \\
\hline $\mathrm{nb}$ & $123 \mid ; 2379 ; 4$ & $25|-5.07030871-2|-5.07030950-2$ & $1.4-10|5.4-10| 7.6-9 \mid 7.1-9$ & 07 & $1.593 \mid \infty$ \\
\hline nb-L1 & $915 \mid ; 2379 ; 797$ & $36|-1.301227061|-1.301227071$ & $4.0-10|3.8-10| 9.0-9 \mid 4.1-9$ & 14 & $3.163 \mid \infty$ \\
\hline nb-L2 & $123 \mid ; 4191 ; 4$ & $19|-1.628971980|-1.628971960$ & $5.9-11|7.3-9| 1.3-9 \mid 3.0-9$ & 10 & $1.683 \mid \infty$ \\
\hline nb-L2-bess & $123 \mid ; 2637 ; 4$ & $17|-1.02569503-1|-1.02569511-1$ & $8.0-12|2.4-9| 7.3-9 \mid 6.4-9$ & 05 & $1.683 \mid \infty$ \\
\hline nql30 & $3680 \mid ; 2700 ; 3602$ & $35|-9.46028479-1|-9.46028497-1$ & $1.5-9|2.2-10| 9.2-9 \mid 6.2-9$ & 03 & $5.403 \mid \infty$ \\
\hline nql60 & $14560 \mid ; 10800 ; 14402$ & $42|-9.35052923-1|-9.35052943-1$ & $1.4-9|1.3-10| 6.9-9 \mid 7.1-9$ & 21 & $2.164 \mid \infty$ \\
\hline nql180 & $130080 \mid ; 97200 ; 129602 ;$ & $61|-9.27728615-1|-9.27728621-1$ & $5.7-9|9.7-12| 7.9-9 \mid 2.0-9$ & 5:07 & $1.945 \mid \infty$ \\
\hline qssp30 & $3691 \mid ; 7564 ; 2$ & $22|-6.496675800|-6.496675750$ & $9.1-9|4.5-10| 6.0-11 \mid 3.5-9$ & 03 & $3.783 \mid \infty$ \\
\hline qssp60 & $14581 \mid ; 29524 ; 2$ & $28|-6.562706380|-6.562706440$ & $6.7-9|3.2-10| 7.4-10 \mid 4.2-9$ & 17 & $1.484 \mid \infty$ \\
\hline qssp 180 & $130141 \mid ; 261364 ; 2$ & $37|-6.639599030|-6.639606910$ & 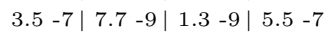 & $4: 48$ & $1.315 \mid \infty$ \\
\hline sched-50-5 & $2527 \mid ; 2477 ; 2502$ & \begin{tabular}{l|l|l}
37 & 2.667300254 & 2.667300094
\end{tabular} & \begin{tabular}{ll|ll|ll|}
5.6 & -8 & 3.1 & -8 & 3.1 & -8 \\
3.0 & -8
\end{tabular} & 02 & $9.766|2.246| 1.35$ \\
\hline sched-100- & $4844 \mid ; 4744 ; 5002$ & $34|1.819151395| 1.818847715$ & $2.2-5|1.1-6| 8.4-5 \mid 8.3-5$ & 04 & $2.957|1.536| 2.65$ \\
\hline sched-100- & $8338 \mid ; 8238 ; 10002$ & \begin{tabular}{l|l|l}
31 & 7.174990255 & 7.173626855
\end{tabular} & $2.1-5|2.3-5| 9.5-5 \mid 9.5-5$ & 09 & $2.598|7.887| 9.65$ \\
\hline sched-200- & $18087 \mid ; 17887 ; 20002$ & $39|1.413612635| 1.413601695$ & $7.1-5|2.9-6| 3.9-6 \mid 3.9-6$ & 39 & $9.077|6.806| 2.25$ \\
\hline sched-50-5 & $2526 \mid ; 2475 ; 2502$ & \begin{tabular}{l|l|l}
28 & 7.852038520 & 0.852038440
\end{tabular} & $3.1-8|7.6-10| 5.0-9 \mid 4.9-9$ & 02 & 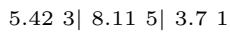 \\
\hline sched-100- & $4843 \mid ; 4742 ; 5002$ & $31|6.716503351| 6.716503071$ & $3.0-8|2.3-10| 2.3-8 \mid 2.0-8$ & 04 & $1.596|5.705| 6.04$ \\
\hline sched-100- & $8337 \mid ; 8236 ; 10002$ & $26 \mid$\begin{tabular}{l|l|l}
2.73307954 & 1 & 2.733078531
\end{tabular} & $2.4-8|4.4-10| 2.1-7 \mid 1.8-7$ & 08 & $1.994|3.707| 1.65$ \\
\hline sched-200- & $18086 \mid ; 17885 ; 20002$ & \begin{tabular}{l|l|l|l}
32 & 5.18119621 & 1 & 5.181196101
\end{tabular} & $2.4-7|1.2-10| 1.1-8 \mid 1.0-8$ & 33 & $6.304|2.766| 7.74$ \\
\hline biggs & $1819 \mid 702 ; ; ;$ & $54|-1.414258403|-1.414258403$ & $6.2-10|9.8-12| 2.1-9 \mid 2.1-9$ & $1: 22$ & $5.794|6.069| 3.57$ \\
\hline buck1 & $36 \mid 49 ; ; 36$ & $17|-1.464191522|-1.464191522$ & $3.1-10|1.5-12| 5.8-10 \mid 5.8-10$ & 01 & $1.862|2.894| 7.72$ \\
\hline buck2 & $144 \mid 193 ;$; 144; & $21|-2.923682642|-2.923682962$ & $5.3-8|4.8-10| 5.4-8 \mid 5.5-8$ & 04 & \begin{tabular}{ll|l|l}
$4.502 \mid$ & $1.316 \mid$ & 1.04
\end{tabular} \\
\hline buck3 & $544 \mid 641 ; ; 544$ & $31|-6.076016912|-6.076060372$ & $3.8-6|2.6-8| 3.5-6 \mid 3.6-6$ & 15 & $1.553|1.947| 2.25$ \\
\hline
\end{tabular}


Table 1: Performance of sdpt3.m. In the table, err = [pinfeas, dinfeas, relgap, relgap2], where relgap2 is the same as relgap but with the numerator replaced by $\left|\langle c, x\rangle-b^{T} y\right|$, and normXZ $=\max \left\{\left\|x^{*}\right\|,\left\|z^{*}\right\|\right\}$. We declare that $g_{P}\left(g_{D}\right)$ is $\infty$ if the computed number is larger than $10^{12}$

\begin{tabular}{|c|c|c|c|c|c|}
\hline problem & $m \mid n_{s} ; n_{q} ; n_{l} ; n_{u}$ & it. | primal obj | dual obj & err & time & $g_{P}\left|g_{D}\right|$ normXZ \\
\hline buck 4 & $1200 \mid 1345 ; ; 1200$ & $37|-4.861419742|-4.861419832$ & $8.0-8|7.2-10| 9.7-9 \mid 8.7-9$ & $1: 34$ & $3.393|1.758| 2.74$ \\
\hline buck5 & $3280 \mid 3521 ; ; 3280$ & $40|-4.361970412|-4.362635092$ & $1.3-6 \mid$\begin{tabular}{ll|ll|ll} 
& 1.7 & -7 & 7.6 & -5 & -5
\end{tabular} & $13: 49$ & $8.693|9.458| 6.14$ \\
\hline cnhil10 & $5005 \mid 220 ; ;$ & $33|0.00000000-16|-1.60338275-4$ & 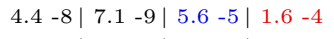 & 44 & | $3.502 \mid 9.84$ \\
\hline cnhil8 & $1716 \mid 120 ; ;$ & $31|0.00000000-16|-4.08644723-6$ & 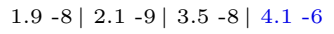 & 06 & | $2.082 \mid 1.34$ \\
\hline cphil10 & $5005 \mid 220 ; ;$ & $10|0.00000000-16|-2.45483691-10$ & $6.7-16|1.0-12| 2.6-10 \mid 2.5-10$ & 22 & $5.002|3.502| 1.41$ \\
\hline cphil12 & $12376 \mid 364 ; ; ;$ & $10|0.00000000-16|-2.43524851-9$ & $0.9-15|2.0-12| 2.5-9 \mid 2.4-9$ & $3: 13$ & $7.852|5.442| 1.51$ \\
\hline G40-mb & 2001 | 2000; ; ; & $21|-2.864322973|-2.864323233$ & $1.9-12|4.2-12| 4.5-8 \mid 4.5-8$ & $16: 23$ & | $4.913 \mid 7.47$ \\
\hline G40mc & $2000 \mid 2000 ; ; ;$ & $18|-5.729579093|-5.729579113$ & $4.8-12|1.0-12| 1.1-9 \mid 1.1-9$ & 4:02 & $2.003|7.833| 6.42$ \\
\hline $\mathrm{G} 48 \mathrm{mc}$ & $3000 \mid 3000 ;$ & $12|-1.200000004|-1.200000004$ & $2.6-13|3.2-12| 3.8-10 \mid 3.7-10$ & $4: 11$ & $3.003|9.003| 3.03$ \\
\hline mater-1 & $103 \mid 220 ; ; 2$ & $15|1.434654402| 1.434654382$ & $1.9-10|2.3-12| 8.1-9 \mid 8.2-9$ & 00 & $1.055|2.222| 1.13$ \\
\hline mater-2 & $423 \mid 1012 ; ; 2$ & $17|1.415918672| 1.415918662$ & $3.7-11|7.6-12| 1.9-9 \mid 1.9-9$ & 01 & $4.725|1.013| 2.43$ \\
\hline mater-3 & $1439 \mid 3586 ; ; 2$ & $18|1.339162572| 1.339162562$ & $1.6-10|2.0-11| 5.3-9 \mid 5.3-9$ & 05 & $1.586|3.593| 4.23$ \\
\hline mater-4 & $4807 \mid 12496 ; ; 2$ & $21|1.342627172| 1.342627162$ & $8.8-11|1.2-11| 5.0-9 \mid 5.0-9$ & 21 & $5.526|1.254| 7.93$ \\
\hline mater-5 & $10143 \mid 26818 ; ; 2$ & $23|1.338016402| 1.338016402$ & $1.0-10|1.4-11| 2.4-9 \mid 2.5-9$ & 53 & $1.187|2.684| 1.14$ \\
\hline mater-6 & $20463 \mid 54626 ; ; 2$ & $29|1.335387152| 1.335387152$ & $4.5-10|4.4-11| 2.7-9 \mid 2.7-9$ & $2: 26$ & $2.407|5.464| 1.64$ \\
\hline neosfbr 12 & $1441 \mid 122 ; ; ;$ & $17|5.293191642| 5.293191582$ & $8.5-11|1.2-12| 5.3-9 \mid 5.3-9$ & 09 & $1.064|8.153| 7.53$ \\
\hline prob-1-2-0 & $100 \mid 200 ; ; ;$ & \begin{tabular}{l|l|l}
25 & $4.3881330910 \mid$ & 4.3881330210
\end{tabular} & $1.4-8|6.8-15| 3.2-8 \mid 7.3-9$ & 24 & $5.192 \mid \infty$ \\
\hline prob-1-2-1 & $100 \mid 200 ; ;$ & $20|-5.5663592210|-5.5663593310$ & $4.4-14|1.0-12| 9.7-9 \mid 9.6-9$ & 20 & $9.492|1.228| 9.32$ \\
\hline prob-2-4-0 & $200 \mid 400 ; ;$ & $27|-6.2807066510|-6.2807074510$ & 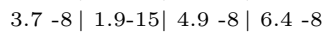 & $3: 35$ & $8.802|\infty \quad| 6.77$ \\
\hline prob-2-4-1 & $200 \mid 400 ; ;$ & $27|9.542810679| 9.542810519$ & $8.0-14|3.2-13| 9.0-9 \mid 8.2-9$ & $8: 49$ & $1.773|9.517| 1.93$ \\
\hline neu1 & $3003 \mid 252 ; ; 2$ & $41|-7.26676865-9|-1.99826835-7$ & $6.9-10|4.2-10| 4.4-7 \mid 1.9-7$ & $8: 24$ & $\infty \quad|\infty \quad| 1.13$ \\
\hline neu1g & $3002 \mid 252 ; ; ;$ & $33|1.250000072| 1.249998472$ & $7.7-10|1.0-9| 1.7-6 \mid 6.4-7$ & 7:09 & | $1.643 \mid 1.86$ \\
\hline neu2 & $3003 \mid 252 ; ; 2$ & $43|-3.96541382-4|-2.23051493-4$ & $4.4-9|1.5-10| 5.0-4 \mid 1.7-4$ & $8: 01$ & $|\infty \quad| 1.55$ \\
\hline neu2c & $3002 \mid 1253 ; ; 2$ & \begin{tabular}{l|l|l|l}
65 & 3.437133814 & 4.389963784
\end{tabular} & $3.2-5 \mid \begin{array}{lllllll} & 1.2 & -6 & 8.4 & -3 & 6.9 & -3\end{array}$ & $25: 45$ & | $1.7410 \mid 9.08$ \\
\hline neu2g & $3002 \mid 252 ; ;$ & \begin{tabular}{l|l|l|l}
33 & 3.410000474 & 3.409987964
\end{tabular} & $4.1-8|3.9-9| 4.1-6 \mid 1.8-6$ & $6: 48$ & $\mid \begin{array}{ll}1.643 \mid & 1.07\end{array}$ \\
\hline neu3 & $7364 \mid 418 ; ; 2$ & $47|3.19225137-10|-2.49655303-9$ & $1.9-12|1.2-12| 6.1-9 \mid 2.8-9$ & $5: 36$ & $|\infty \quad| 1.53$ \\
\hline neu3g & $8007 \mid 462 ; ; ;$ & $49|1.58672362-5|-9.13182744-5$ & $5.4-15|3.6-6| 2.4-4 \mid 1.1-4$ & $7: 00$ & | $2.553 \mid 1.111$ \\
\hline rendl1-600 & $601 \mid 600 ; ;$ & $15|-5.579687054|-5.579687064$ & $1.9-10|5.5-11| 2.1-9 \mid 1.3-9$ & 36 & 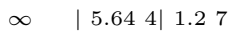 \\
\hline r1-6-0 & $601 \mid 600 ; ;$ & $15|-5.579687054|-5.579687064$ & $1.9-10|5.5-11| 2.1-9 \mid 1.3-9$ & 44 & $|5.644| 1.27$ \\
\hline $\mathrm{r} 1-6-1$ & $601 \mid 600 ; ;$ & $14|-5.580439224|-5.580439244$ & $4.3-11|1.1-12| 2.3-9 \mid 2.2-9$ & 38 & $3.605|5.644| 2.73$ \\
\hline r1-6-1e-6 & $601 \mid 600 ; ;$ & $19|-5.579687314|-5.579687314$ & $1.1-8|1.9-11| 1.3-10 \mid 2.8-10$ & 56 & | $5.644 \mid 7.75$ \\
\hline $\operatorname{rose} 13$ & $2379 \mid 105 ; ;$ & $31|1.200000051| 1.199999991$ & $5.8-10|1.9-11| 4.9-9 \mid 2.4-8$ & $1: 13$ & | $1.192 \mid 1.14$ \\
\hline rose15 & $3860 \mid 135 ; ; 2$ & $47|1.40096194-7|-2.71682585-8$ & $4.4-8|6.5-12| 2.6-8 \mid 1.7-7$ & $8: 07$ & $|\infty \quad| 7.12$ \\
\hline sdmint3 & $5255 \mid 379 ; 5255 ;$ & $37|-1.28667151 \quad 1|-1.285124391$ & $7.1-7|2.7-7| 2.9-4 \mid 5.8-4$ & $30: 52$ & | $8.864 \mid 2.45$ \\
\hline shmup1 & $16 \mid 81 ; ; 32$ & $15|-1.884148292|-1.884148332$ & $1.8-11|1.7-12| 9.6-9 \mid 9.5-9$ & 01 & $1.502|1.406| 3.03$ \\
\hline shmup2 & $200 \mid 881 ; ; 400$ & $32|-3.462426793|-3.462426833$ & $2.9-9|1.1-12| 5.9-9 \mid 5.4-9$ & 32 & $1.353|3.777| 2.55$ \\
\hline shmup3 & $420 \mid 1801 ; ; 840$ & $37|-2.098837863|-2.098837883$ & $5.8-9|8.6-13| 6.0-9 \mid 5.5-9$ & $3: 28$ & $2.743|2.917| 3.35$ \\
\hline shmup 4 & $800 \mid 3361 ; ; 1600$ & $55|-7.992551433|-7.992551543$ & $8.5-9|1.2-12| 8.7-9 \mid 7.4-9$ & $22: 20$ & $5.103|6.737| 4.25$ \\
\hline taha1a & $3002 \mid 1680 ; ;$ & $27|-9.99980977-1|-1.000075770$ & $6.2-5|2.9-10| 3.6-5 \mid 3.2-5$ & $11: 30$ & | $1.07 \quad 10 \mid 1.47$ \\
\hline taha1b & $8007 \mid 1606 ; ; 3$ & $33|-7.73287084-1|-7.73322140-1$ & $3.2-11|1.1-7| 1.4-5 \mid 1.4-5$ & $22: 00$ & $\infty \quad|2.525| 1.210$ \\
\hline trto1 & $36 \mid 25 ; ; 36$ & $13|-1.104500003|-1.104500003$ & $7.3-11|1.5-12| 1.4-9 \mid 1.4-9$ & 00 & $1.112|3.144| 4.83$ \\
\hline trto2 & $144 \mid 97 ; ; 144$ & $21|-1.279999614|-1.280000084$ & $7.6-7 \mid$\begin{tabular}{lll|ll|ll} 
& 6.0 & -9 & 1.9 & -7 & 1.8 & -7
\end{tabular} & 02 & $2.972|1.376| 1.05$ \\
\hline trto3 & $544 \mid 321 ; ; 544$ & $25|-1.279999124|-1.280000524$ & 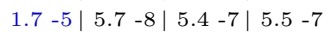 & 08 & $1.053|9.696| 2.75$ \\
\hline trto 4 & $1200 \mid 673 ; ; 1200$ & $33|-1.276580744|-1.276582884$ & $3.8-6|8.8-8| 9.2-7 \mid 8.4-7$ & 46 & $2.293|3.747| 5.35$ \\
\hline trto5 & $3280 \mid 1761 ; ; 3280$ & $35|-1.279969874|-1.280000144$ & $8.9-5 \mid \begin{array}{lllllll} & 6.7 & -7 & 1.3 & -5 & 1.2 & -5\end{array}$ & $10: 45$ & $5.993|2.398| 1.36$ \\
\hline vibra1 & $36 \mid 49 ; ; 36$ & $13|-4.081901231|-4.081901241$ & $1.5-11|1.0-12| 1.8-9 \mid 1.8-9$ & 00 & $1.852|1.634| 1.03$ \\
\hline vibra2 & $144 \mid 193 ; ; 144$ & $25|-1.660153342|-1.660153652$ & $3.9-8|7.3-10| 1.0-7 \mid 9.2-8$ & 05 & $4.502|1.346| 3.34$ \\
\hline vibra3 & $544 \mid 641 ; ; 544$ & $32|-1.726128062|-1.726130802$ & 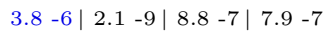 & 15 & $1.553|1.627| 1.55$ \\
\hline vibra4 & $1200 \mid 1345 ; ; 1200$ & $35|-1.276581374|-1.276582644$ & 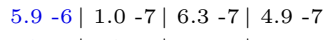 & $1: 32$ & $3.393|7.437| 5.35$ \\
\hline vibra5 & $3280 \mid 3521 ; ; 3280$ & $66|-1.659006462|-1.659034632$ & $9.7-6 \mid$\begin{tabular}{ll|ll|ll} 
& 1.7 & -8 & 9.3 & -6 & 8.5
\end{tabular} & $35: 15$ & $8.693|1.129| 5.45$ \\
\hline yalsdp & $5051 \mid 300 ; ; ;$ & $13|-1.792126720|-1.792126750$ & $2.1-10|2.1-12| 5.9-9 \mid 6.4-9$ & $8: 42$ & $2.593|4.993| 3.92$ \\
\hline checker-1. & $3970 \mid 3970 ; ;$ & $23|3.303884563| 3.303884543$ & $1.2-12|1.0-12| 2.9-9 \mid 2.9-9$ & $17: 12$ & $3.973|1.824| 2.93$ \\
\hline foot & 2209 | 2208; ; ; & $25|-5.852939685|-5.852981715$ & $3.2-6|7.9-8| 3.6-6 \mid 3.6-6$ & $13: 10$ & $|5.905| 3.78$ \\
\hline hand & $1297 \mid 1296 ; ; ;$ & $17|-2.474777904|-2.474777914$ & $1.1-11|7.6-11| 1.9-9 \mid 1.9-9$ & $4: 37$ & $2.484 \mid 2.17$ \\
\hline inc-600 & $2515 \mid 600 ; ; 2514$ & $32|-6.68278108-1|-6.68550231-1$ & $4.5-8 \mid$\begin{tabular}{lllll|ll} 
& 1.7 & -9 & 1.2 & -4 & 1.2 & -4
\end{tabular} & $2: 33$ & $|2.675| 2.47$ \\
\hline inc- 1200 & $5175 \mid 1200 ; ; 5174$ & $44|-1.157387630|-1.157470450$ & $5.0-8|1.6-9| 3.2-5 \mid 2.5-5$ & $10: 25$ & $6.255 \mid 9.67$ \\
\hline tiger-text & $1802 \mid 1801 ; ;$ & $36|3.448425402| 3.443608152$ & $7.3-8 \mid \begin{array}{llllllll} & 1.6 & -8 & 1.0 & -3 & 7.0 & -4\end{array}$ & $6: 59$ & | $3.333 \mid 1.09$ \\
\hline butcher & $6434 \mid 330 ; ; 22512$ & $53|-1.399998951|-1.399999991$ & $3.0-6|6.4-10| 1.4-7 \mid 3.6-7$ & $34: 56$ & $2.284 \mid \infty$ \\
\hline rabmo & $5004 \mid 220 ; ; 6606$ & $37|-3.727252670|-3.727246670$ & $4.0-7 \mid \begin{array}{lllllll} & 7.5 & -8 & 8.7 & -7 & 7.1 & -7\end{array}$ & $6: 40$ & $6.833 \mid \infty$ \\
\hline chs -500 & $9974 \mid 4980 ; ;$ & $24|7.18222801-10|-6.43232980-9$ & $1.2-15|1.0-12| 7.3-9 \mid 7.2-9$ & 12 & $7.35 \quad 7 \mid \begin{array}{lll}6.97 & 3 \mid 2.21\end{array}$ \\
\hline nonc-500 & $4990 \mid 2998 ; ; ;$ & $23 \mid 6.25761441-2$ | 6.25594864-2 & $5.0-10|5.9-11| 1.5-5 \mid 1.5-5$ & 03 & $2.264|6.003| 6.61$ \\
\hline ros -500 & $4988 \mid 2992 ; ; ;$ & $17|2.494999440| 2.494999390$ & $8.2-10|3.4-12| 7.1-9 \mid 7.8-9$ & 02 & $3.185|4.493| 1.32$ \\
\hline
\end{tabular}


Table 1: Performance of sdpt3.m. In the table, err = [pinfeas, dinfeas, relgap, relgap2], where relgap2 is the same as relgap but with the numerator replaced by $\left|\langle c, x\rangle-b^{T} y\right|$, and normXZ $=\max \left\{\left\|x^{*}\right\|,\left\|z^{*}\right\|\right\}$. We declare that $g_{P}\left(g_{D}\right)$ is $\infty$ if the computed number is larger than $10^{12}$.

\begin{tabular}{|c|c|c|c|c|c|}
\hline problem & $m \mid n_{s} ; n_{q} ; n_{l} ; n_{u}$ & it. | primal obj | dual obj & err & time & $g_{P}\left|g_{D}\right|$ normXZ \\
\hline fp210 & $1000 \mid 176 ; ; ; 66$ & $25|3.75000000-1| 3.75000001-1$ & $2.9-11|1.3-9| 1.2-10 \mid 7.4-10$ & 09 & | 1.51 \\
\hline fp22 & $14 \mid 15 ; ; ;$ & $12|-7.999999990|-8.000000020$ & $2.1-11|1.3-11| 2.1-9 \mid 1.7-9$ & 00 & $1.142|1.125| 6.71$ \\
\hline fp23 & $209 \mid 119 ; ; ;$ & \begin{tabular}{l|l|l|}
27 & 2.130000002 & 2.130000002
\end{tabular} & 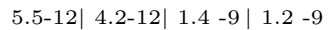 & 01 & $\mid \begin{array}{lll}2.95 & 3 \mid 2.35\end{array}$ \\
\hline fp24 & $2379 \mid 595 ; ; ;$ & $22|1.950000002| 1.949999982$ & $1.7-11|1.0-12| 7.8-9 \mid 7.3-9$ & 52 & $8.153 \mid 9.64$ \\
\hline fp25 & $209 \mid 133 ; ; ;$ & $18|1.100000001| 1.100000001$ & $9.9-13|9.9-13| 1.4-9 \mid 1.3-9$ & 01 & $5.943 \mid 6.93$ \\
\hline fp26 & $1000 \mid 407 ; ; ;$ & $22|2.680146322| 2.680146302$ & $1.5-10|1.1-11| 5.5-9 \mid 4.5-9$ & 09 & | $5.185 \mid 3.35$ \\
\hline $\mathrm{fp} 27$ & $1000 \mid 341 ; ; ;$ & $22|3.900000021| 3.899999961$ & $7.4-11|6.4-12| 7.5-9 \mid 6.5-9$ & 08 & $|2.435| 8.45$ \\
\hline fp32 & $3002 \mid 1155 ; ;$ & $43|-7.049183820|-7.049831210$ & $5.4-4|6.6-10| 6.1-5 \mid 4.3-5$ & $7: 38$ & 1.18 \\
\hline fp33 & $125 \mid 117 ; ; ;$ & $42|-1.012659054|-1.012660444$ & $1.7-7|2.3-9|$\begin{tabular}{lll|}
8 & 8.6 & -7 \\
6.9 & -7
\end{tabular} & 01 & $\mid$\begin{tabular}{ll|l}
3.30 & $10 \mid$ & 3.49
\end{tabular} \\
\hline fp34 & $209 \mid 140 ; ;$ & $22|1.720000002| 1.720000002$ & $7.5-13|1.0-12| 7.7-10 \mid 6.4-10$ & 01 & | $1.084 \mid 2.64$ \\
\hline fp35 & $164 \mid 195 ; ;$ & $19|3.999999990| 3.999999990$ & $1.3-9|2.2-12| 2.2-9 \mid 6.7-10$ & 03 & $|2.855| 5.44$ \\
\hline $\mathrm{fp} 410$ & $14 \mid 18 ; ; ; 1$ & $17|1.673889321| 1.673889321$ & $2.9-9|3.1-9| 3.1-10 \mid 3.2-10$ & 00 & 1.61 \\
\hline fp42 & $6 \mid 10 ; ; ;$ & $10|7.587312370| 7.587312360$ & $2.2-12|2.5-11| 8.5-10 \mid 7.8-10$ & 00 & $7.191|9.781| 5.51$ \\
\hline $\mathrm{fp} 43$ & $50 \mid 76 ; ; ;$ & $16|6.635000602| 6.635001242$ & $2.7-9|1.9-10| 4.2-10 \mid 4.9-8$ & 01 & $3.443 \mid \infty$ \\
\hline $\mathrm{fp} 44$ & $6 \mid 10 ; ; ;$ & \begin{tabular}{l|l|l}
21 & 4.436716882 & 2.436717042
\end{tabular} & $4.8-9|2.4-10| 2.4-9 \mid 1.7-8$ & 00 & $|1.223| 6.84$ \\
\hline $\mathrm{fp} 45$ & $4 \mid 7 ; ;$; & $12|2.32020677-9|-8.03431099-10$ & $8.1-11|2.5-11| 4.2-9 \mid 3.1-9$ & 00 & $8.301|2.961| \begin{array}{lll}1.9 & 1\end{array}$ \\
\hline fp46 & $27 \mid 22 ; ; ;$ & $20|5.45358326-10|-2.96042821-9$ & $2.8-11|1.1-11| 3.9-9 \mid 3.5-9$ & 00 & | $1.492 \mid 2.33$ \\
\hline $\mathrm{fp} 47$ & $6 \mid 10 ; ;$ & $13 \mid 2.429999952$ | 2.429999992 & $1.6-9|2.2-9| 9.3-9 \mid 8.5-9$ & 00 & $1.523|7.991| 9.62$ \\
\hline fp48 & $4 \mid 7 ; ;$ & $9|7.500000010| 7.499999990$ & $4.7-11|4.3-11| 9.1-10 \mid 8.5-10$ & 00 & $9.681|2.961| 1.51$ \\
\hline fp49 & $14 \mid 18 ; ; ; 1$ & $17|1.673889321| 1.673889321$ & $2.9-9|3.1-9| 3.1-10 \mid 3.2-10$ & 00 & | 1.61 \\
\hline 11 & $14 \mid 6 ; ;$ & $9|4.92634655-1| 4.92634654-1$ & $9.9-13|1.5-12| 5.6-10 \mid 5.5-10$ & 00 & $1.491|9.000| 5.70$ \\
\hline 12 & $14 \mid 6 ; ; ;$ & \begin{tabular}{l|l|l|l|l}
8 & 1.14580631 & 1 & 1.14580631 & 1
\end{tabular} & $2.4-11|3.9-10| 1.4-9 \mid 1.1-9$ & 00 & $6.831|9.000| 1.41$ \\
\hline 14 & $152 \mid 45 ; ;$ & $21|3.70371192-2| 3.70377081-2$ & $7.3-11|4.9-11| 5.1-7 \mid 5.5-7$ & 01 & $\infty \quad|1.064| 7.93$ \\
\hline 15 & $14 \mid 15 ; ; ;$ & $12|-7.999999990|-8.000000020$ & $2.1-11|1.3-11| 2.1-9 \mid 1.7-9$ & 00 & $1.142|1.125| 6.71$ \\
\hline $5 \mathrm{n}$ & $31 \mid 26 ; ; ;$ & $9|2.240000010| 2.239999980$ & $9.1-11|1.0-11| 5.4-9 \mid 5.3-9$ & 00 & $2.821|2.601| \begin{array}{lll}\mid & 1.1 & 1\end{array}$ \\
\hline a12 & $793 \mid 79 ; ;$ & $12|2.100000001| 2.100000001$ & $1.1-11|1.5-12| 1.3-9 \mid 1.2-9$ & 02 & $1.002|7.901| 4.41$ \\
\hline aw29 & $465 \mid 130 ; ; ;$ & $11|3.000000000| 3.000000000$ & $4.7-12|2.9-11| 4.5-10 \mid 4.4-10$ & 07 & 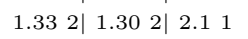 \\
\hline c5 & $31 \mid 26 ; ; ;$ & $8|1.500000000| 1.500000000$ & $3.0-12|3.4-12| 4.2-10 \mid 4.2-10$ & 00 & $2.751|2.601| 8.60$ \\
\hline fp1131 & $847 \mid 176 ; ; ;$ & $11|4.500000010| 4.499999950$ & $3.9-11|1.0-12| 6.3-9 \mid 6.2-9$ & 17 & $1.812|1.762| 6.21$ \\
\hline fp1132 & $847 \mid 176 ; ; ;$ & $12|1.550000001| 1.549999991$ & $1.0-11|7.8-12| 4.6-9 \mid 4.6-9$ & 18 & $1.922|1.762| 1.42$ \\
\hline fp1133 & $847 \mid 176 ; ; ;$ & $12 \mid$\begin{tabular}{ll|l}
1.75000000 & 1 & 1.749999991
\end{tabular} & $8.2-12|4.4-12| 2.2-9 \mid 2.2-9$ & 18 & $1.942|1.762| 1.42$ \\
\hline fp1134 & $847 \mid 176 ; ;$ & \begin{tabular}{l|l|l}
12 & 1.950000001 & 1.949999981
\end{tabular} & $3.8-11|1.0-12| 7.0-9 \mid 6.9-9$ & 18 & $1.962|1.762| 1.42$ \\
\hline fp1135 & $847 \mid 176 ; ; ;$ & $12|2.200000001| 2.199999991$ & $9.4-12|3.9-12| 1.9-9 \mid 1.8-9$ & 22 & \begin{tabular}{ll|l|l|l}
1.98 & $2 \mid$ & 1.76 & 2 & 5.81
\end{tabular} \\
\hline fp1136 & $847 \mid 176 ; ; ;$ & $12|1.450000001| 1.449999991$ & $9.0-12|3.8-12| 3.5-9 \mid 3.5-9$ & 19 & $1.912|1.762| 1.42$ \\
\hline fp1137 & $847 \mid 176 ; ; ;$ & $12 \mid$\begin{tabular}{ll|l}
1.65000000 & 1 & 1.649999991
\end{tabular} & $7.8-12|7.5-12| 3.0-9 \mid 3.0-9$ & 19 & $1.932|1.762| 1.12$ \\
\hline fp1138 & $847 \mid 176 ; ;$ & $12|1.750000001| 1.749999971$ & $4.6-11|1.0-12| 8.6-9 \mid 8.4-9$ & 18 & $1.942|1.762| 1.42$ \\
\hline fp1139 & $847 \mid 176 ; ; ;$ & $12|2.300000001| 2.299999981$ & $7.2-12|6.6-12| 4.7-9 \mid 4.7-9$ & 18 & \begin{tabular}{ll|ll|l}
1.99 & $2 \mid$ & 1.76 & 2 & 8.51
\end{tabular} \\
\hline $\mathrm{k} 5$ & $31 \mid 31 ; ; ;$ & $8 \mid$\begin{tabular}{l|l|l} 
& 1.00000000 & 0
\end{tabular} & $8.5-13|2.6-12| 1.1-9 \mid 1.1-9$ & 00 & $3.201|3.101| 7.00$ \\
\hline $\mathrm{p} 10$ & $847 \mid 176 ; ;$ & $11|4.500000010| 4.499999950$ & $4.1-11|1.0-12| 6.3-9 \mid 6.2-9$ & 16 & \begin{tabular}{ll|ll|ll}
1.81 & $2 \mid$ & 1.76 & $2 \mid$ & 6.21
\end{tabular} \\
\hline bifur & $454 \mid 84 ; ; ; 1661$ & $28|-3.37301696-1|-3.37301694-1$ & $2.2-9|1.4-9| 3.6-9 \mid 9.1-10$ & 07 & $3.413|\infty \quad| 4.11$ \\
\hline boom & $3002 \mid 210 ; ; ; 8764$ & $36|-3.237072452|-3.237072452$ & $4.8-9|7.0-10| 1.0-10 \mid 2.8-10$ & $4: 57$ & $1.774|6.7211| 2.82$ \\
\hline brown & $461 \mid 56 ; ; ; 925$ & $27|2.09326284-10| 0.00000000-16$ & $2.2-11|3.6-9| 2.1-10 \mid 2.1-10$ & 04 & | 5.71 \\
\hline butcher & $6434 \mid 330 ; ; ; 11256$ & $59|-1.399999991|-1.400000001$ & $2.1-6|7.8-12| 9.4-10 \mid 2.1-9$ & $30: 44$ & $2.284|1.918| 2.73$ \\
\hline camera1s & $209 \mid 28 ; ; ; 168$ & $44|-1.786865144|-1.786865134$ & $1.9-6|3.8-11| 3.6-11 \mid 4.8-9$ & 01 & $3.642 \mid \infty$ \\
\hline caprasse & $209 \mid 35 ; ; ; 60$ & $16|-2.36780177-1|-2.36780177-1$ & $1.2-9|6.1-10| 7.2-12 \mid 1.5-10$ & 02 & $1.552 \mid \infty$ \\
\hline cdpm5 & $125 \mid 21 ; ; ; 5$ & $14|4.50956437-12| 6.11518578-9$ & $2.8-9|5.0-9| 1.2-10 \mid 6.1-9$ & 00 & $3.101 \mid \infty$ \\
\hline chemequ & $461 \mid 56 ; ; ; 525$ & $16|-2.778203377|-1.627044757$ & dual infeasible & 04 & \\
\hline chemequs & $125 \mid 21 ; ; ; 45$ & $9|-5.753128728|-6.249334576$ & dual infeasible & 00 & \\
\hline $\operatorname{cohn} 2$ & $209 \mid 35 ; ; ; 4$ & $36|4.57937090-9| 1.29980577-7$ & $3.8-7|6.9-7| 1.4-7 \mid 1.3-7$ & 06 & $4.301 \mid \infty$ \\
\hline cohn3 & $209 \mid 35 ; ; ; 4$ & $35|7.86066516-9| 8.98299495-9$ & $2.1-6|3.4-7| 2.3-7 \mid 1.1-9$ & 06 & $4.301 \mid \infty$ \\
\hline comb3000 & $1000 \mid 66 ; ; ; 595$ & $25|-4.80138795-10| \quad 1.17568288-9$ & $6.3-12|4.8-9| 3.2-10 \mid 1.7-9$ & 09 & $1.263|4.889| 6.50$ \\
\hline conform 1 & $83 \mid 20 ; ; ; 30$ & $9|-1.697231997|-1.793531396$ & dual infeasible & 01 & \\
\hline conform 3 & $285 \mid 56 ; ; ; 630$ & $21|1.01682942-13| 0.00000000-16$ & $2.0-13|3.6-10| 2.2-11 \mid 1.0-13$ & 02 & | 2.50 \\
\hline conform 4 & $454 \mid 84 ; ; ; 1890$ & $21|4.05675465-11| 0.00000000-16$ & $3.4-11|4.5-9| 4.4-10 \mid 4.1-11$ & 06 & $\infty \quad \mid \infty$ \\
\hline $\operatorname{des} 22-24$ & $1000 \mid 66 ; ; ; 660$ & $37|-6.741663653|-6.741663653$ & $2.0-8|4.1-12| 2.1-13 \mid 1.4-12$ & 14 & $\begin{array}{llll}1.39 & 3 \mid 2.27 & 10 \mid & 6.73\end{array}$ \\
\hline discret 3 & $44 \mid 9 ; ; ; 8$ & $24|-3.700331091|-3.700330911$ & $2.4-7|1.5-8| 4.0-10 \mid 2.4-8$ & 00 & $2.501 \mid \infty$ \\
\hline eco5 & $461 \mid 56 ; ; ; 525$ & $26|-1.204633113|-1.204633113$ & $3.2-9|5.8-9| 1.4-10 \mid 1.2-10$ & 03 & $1.113|5.2110| 1.23$ \\
\hline eco6 & $923 \mid 84 ; ; ; 924$ & $37|-1.002815594|-1.002815594$ & $6.5-9|1.3-9| 1.4-10 \mid 9.8-11$ & 14 & \begin{tabular}{ll|ll|l}
1.93 & 3 & 5.76 & $10 \mid$ & 1.04
\end{tabular} \\
\hline eco7 & $1715 \mid 120 ; ; ; 1512$ & $37|-3.915310473|-3.915310473$ & $5.3-9|2.8-9| 1.7-10 \mid 4.2-12$ & 47 & \begin{tabular}{ll|ll|l}
3.14 & 3.28 & 11 & 3.93
\end{tabular} \\
\hline eco8 & $3002 \mid 165 ; ; ; 2340$ & $37|-5.820384183|-5.820384183$ & $5.2-9|3.3-9| 1.2-10 \mid 2.6-11$ & $4: 19$ & \begin{tabular}{ll|ll|l}
4.85 & 3 & 2.96 & $11 \mid$ & 5.83
\end{tabular} \\
\hline fourbar & $69 \mid 15 ; ; ; 4$ & $14|1.01084283-12| 1.17629630-9$ & $1.9-10|7.4-10| 1.9-11 \mid 1.2-9$ & 00 & $2.301 \mid \infty$ \\
\hline geneig & $923 \mid 84 ; ; ; 546$ & $25|-2.526630140|-2.526630140$ & $2.2-10|2.3-9| 3.6-10 \mid 2.5-10$ & 09 & \begin{tabular}{ll|ll|ll}
1.18 & 3 & 1.38 & $11 \mid$ & 8.9 & 0
\end{tabular} \\
\hline
\end{tabular}


Table 1: Performance of sdpt3.m. In the table, err = [pinfeas, dinfeas, relgap, relgap2], where relgap2 is the same as relgap but with the numerator replaced by $\left|\langle c, x\rangle-b^{T} y\right|$, and normXZ $=\max \left\{\left\|x^{*}\right\|,\left\|z^{*}\right\|\right\}$. We declare that $g_{P}\left(g_{D}\right)$ is $\infty$ if the computed number is larger than $10^{12}$.

\begin{tabular}{|c|c|c|c|c|c|c|}
\hline problem & $m \mid n_{s} ; n_{q} ; n_{l} ; n_{u}$ & it. | primal obj | dual obj & err & time & $g_{P}\left|g_{D}\right|$ & normXZ \\
\hline heart & $3002 \mid 165 ; ; ; 4320$ & $32|-8.709274201|-8.709274211$ & $7.2-9|3.7-9| 2.8-10 \mid 4.9-10$ & $3: 41$ & $8.813 \mid 2.79$ & $11 \mid 1.42$ \\
\hline i1 & $1000 \mid 66 ; ; ; 10$ & $17|-1.667752720|-1.667752690$ & $3.2-9|5.1-9| 7.8-10 \mid 6.2-9$ & 06 & $8.601 \mid \infty$ & 9.40 \\
\hline ipp & $494 \mid 45 ; ; ; 360$ & $22|-1.311588531|-1.311588531$ & \begin{tabular}{ll|l|l|l|}
$4.2-9$ & $5.8-9$ & $2.4-10 \mid 3.1-11$
\end{tabular} & 02 & $7.652 \mid \infty$ & | 1.71 \\
\hline katsura5 & $209 \mid 28 ; ; ; 168$ & $20|-8.16044579-2|-8.16044568-2$ & $2.3-10|1.3-9| 9.7-11 \mid 9.1-10$ & 01 & $3.642 \mid \infty$ & 5.40 \\
\hline kinema & $714 \mid 55 ; ; ; 495$ & $37|-4.196839634|-4.196839634$ & $1.2-7|2.9-12| 1.7-12 \mid 2.6-13$ & 07 & $1.053 \mid \infty$ & 4.24 \\
\hline ku10 & $1000 \mid 66 ; ; ; 660$ & $34|-7.139000003|-7.139000003$ & $1.5-7|3.8-10| 2.8-11 \mid 1.5-10$ & 12 & $1.393 \mid \infty$ & | 7.13 \\
\hline lorentz & $69 \mid 15 ; ; ; 60$ & $17|-5.000000000|-4.999999970$ & $8.1-10|6.3-9| 7.7-11 \mid 2.0-9$ & 00 & $1.352 \mid \infty$ & | 6.10 \\
\hline manocha & $90 \mid 28 ; ; ; 42$ & $36|-2.46011903-1|-2.46013262-1$ & $3.0-5|7.5-9| 3.3-8 \mid 9.1-7$ & 02 & $1.122 \mid \infty$ & 1.43 \\
\hline noon3 & $83 \mid 20 ; ; ; 30$ & $18|-2.086950331|-2.086950331$ & $1.1-9|1.0-9| 2.3-11 \mid 4.7-12$ & 01 & $8.001 \mid \infty$ & | 2.61 \\
\hline noon4 & $209 \mid 35 ; ; ; 60$ & $18|-1.712837591|-1.712837591$ & $9.6-10|1.1-9| 2.6-11 \mid 7.3-11$ & 01 & $1.552 \mid \infty$ & 1.91 \\
\hline noon5 & $461 \mid 56 ; ; ; 105$ & $18|-1.585242431|-1.585242431$ & $7.5-10|1.5-9| 1.9-11 \mid 3.9-11$ & 02 & $2.662 \mid \infty$ & 1.71 \\
\hline proddeco & $69 \mid 15 ; ; ; 4$ & $18|1.29678458-11| 4.63789541-10$ & $1.8-10|8.9-10| 1.5-10 \mid 4.5-10$ & 00 & $2.301 \mid \infty$ & 3.30 \\
\hline puma & $3002 \mid 165 ; ; ; 8280$ & $31|-3.052994891|-3.052994891$ & $2.9-9|9.8-11| 5.5-11 \mid 3.8-10$ & 3:38 & $1.674 \mid 9.28$ & $11 \mid 3.41$ \\
\hline quadfor2 & $209 \mid 35 ; ; ; 270$ & $19|-6.185185180|-6.185185180$ & $1.3-9|7.9-10| 4.1-11 \mid 1.1-10$ & 03 & $5.752 \mid \infty$ & | 1.91 \\
\hline quadgrid & $461 \mid 56 ; ; ; 505$ & $17|-1.088015887|-8.429373246$ & dual infeasible & 02 & & \\
\hline rabmo & $5004 \mid 220 ; ; ; 3303$ & $42|-3.727253050|-3.727251760$ & $6.7-8|1.7-8| 1.1-7 \mid 1.5-7$ & 7:09 & $6.833 \mid \infty$ & 4.52 \\
\hline $\mathrm{rbpl}$ & $923 \mid 84 ; ; ; 546$ & $26|-7.940633770|-7.940633770$ & $2.6-9|7.5-10| 6.7-11 \mid 2.3-10$ & 10 & $1.183 \mid \infty$ & | 3.11 \\
\hline redeco5 & $20 \mid 6 ; ; ; 5$ & $13|-2.53906248-1|-2.53906249-1$ & $2.3-9|7.6-10| 4.6-11 \mid 8.9-10$ & 00 & $1.601 \mid \infty$ & 2.40 \\
\hline redeco6 & $27 \mid 7 ; ; ; 6$ & $13|-2.01599999-1|-2.01599999-1$ & $1.5-9|7.7-10| 2.8-11 \mid 4.2-10$ & 00 & $1.901 \mid \infty$ & 2.60 \\
\hline redeco7 & $35 \mid 8 ; ; ; 7$ & $13|-1.67438272-1|-1.67438268-1$ & $7.4-11|5.9-9| 9.1-11 \mid 2.3-9$ & 00 & $2.201 \mid \infty$ & 2.70 \\
\hline redeco 8 & $44 \mid 9 ; ; ; 8$ & $13|-1.43273635-1|-1.43273635-1$ & $1.6-9|6.1-10| 1.5-11 \mid 3.0-10$ & 00 & $2.501 \mid \infty$ & 2.90 \\
\hline rediff3 & $9 \mid 4 ; ; ; 3$ & $16|7.61297271-13| 3.66436581-9$ & $1.1-9|2.5-9| 8.4-12 \mid 3.7-9$ & 00 & $1.001 \mid \infty$ & | 1.70 \\
\hline rose & $679 \mid 120 ; ; ; 2281$ & $53|-1.743790540|-1.743763460$ & $2.5-4|4.7-9| 1.2-4 \mid 6.0-6$ & 29 & $4.683 \mid \infty$ & 2.85 \\
\hline s9-1 & $494 \mid 45 ; ; ; 360$ & $22|-4.273695630|-4.273695640$ & $5.5-9|1.6-9| 4.3-10 \mid 1.4-9$ & 02 & $7.652 \mid \infty$ & | 9.40 \\
\hline sendra & $65 \mid 21 ; ; ; 12$ & $21|-2.376875421|-2.376875421$ & $5.9-9|4.4-10| 1.6-11 \mid 9.2-11$ & 01 & $4.501 \mid \infty$ & 4.31 \\
\hline solotarev & $69 \mid 15 ; ; ; 32$ & $18|-5.889613330|-5.889613330$ & $5.7-10|3.1-10| 7.7-12 \mid 9.8-11$ & 01 & $7.901 \mid \infty$ & 1.11 \\
\hline stewart1 & $714 \mid 55 ; ; ; 495$ & $28|-8.765852780|-8.765852780$ & $8.6-9|2.0-10| 1.1-11 \mid 3.0-11$ & 05 & $1.053 \mid \infty$ & 2.11 \\
\hline stewart2 & $1819 \mid 91 ; ; ; 910$ & $28|-1.275313851|-1.275313861$ & $9.9-9|7.6-10| 2.3-10 \mid 1.8-9$ & 34 & $1.913 \mid \infty$ & 1.81 \\
\hline trinks & $209 \mid 28 ; ; ; 141$ & $27|-2.43523491-1|-2.43523224-1$ & $6.2-9|8.8-9| 1.0-8 \mid 1.8-7$ & 01 & $3.102 \mid \infty$ & 2.81 \\
\hline visasoro & $44 \mid 9 ; ; ; 8$ & $15|1.73075654-13| 1.66876819-9$ & $1.0-9|6.8-10| 4.7-12 \mid 1.7-9$ & 00 & $2.501 \mid \infty$ & 2.80 \\
\hline wood & $69 \mid 15 ; ; ; 32$ & $18|-6.64233344-2|-6.64233342-2$ & $1.4-11|1.4-9| 1.5-11 \mid 1.7-10$ & 01 & $7.901 \mid \infty$ & | 3.70 \\
\hline wright & $20 \mid 6 ; ; ; 5$ & $17|-2.000000001|-1.999999991$ & $2.0-9|6.2-9| 1.3-9 \mid 1.1-9$ & 00 & $1.601 \mid \infty$ & 2.11 \\
\hline nql30o & $3680 \mid ; 2700 ; 3602$ & $37|-9.46028486-1|-9.46028499-1$ & $1.0-9|1.6-10| 7.1-9 \mid 4.4-9$ & 04 & $5.403 \mid \infty$ & 5.41 \\
\hline nql60o & $14560 \mid ; 10800 ; 14402$ & $42|-9.35052921-1|-9.35052943-1$ & $1.4-9|1.4-10| 8.2-9 \mid 7.6-9$ & 27 & $2.164 \mid \infty$ & 1.12 \\
\hline nq190o & $32640 \mid ; 24300 ; 32402$ & $49|-9.31383156-1|-9.31383163-1$ & $5.1-10|3.2-11| 6.7-9 \mid 2.4-9$ & 1:01 & $4.864 \mid \infty$ & 1.62 \\
\hline nql120o & $57920 \mid ; 43200 ; 57602$ & $51|-9.29550226-1|-9.29550233-1$ & $4.9-9|2.5-11| 7.4-9 \mid 2.4-9$ & 2:03 & $8.644 \mid \infty$ & 2.12 \\
\hline nql180o & $130080 \mid ; 97200 ; 129602$ & $60|-9.27728615-1|-9.27728621-1$ & $5.1-9|1.0-11| 7.6-9 \mid 2.0-9$ & 5:07 & $1.945 \mid \infty$ & 3.22 \\
\hline qs $30 \circ$ & $1861 \mid ; 3844 ; 2$ & $22|-6.295315770|-6.295315620$ & $2.6-8|1.2-9| 2.0-12 \mid 1.1-8$ & 02 & $1.923 \mid \infty$ & 4.41 \\
\hline qs 600 & $7321 \mid ; 14884 ; 2$ & $28|-6.382104310|-6.382103770$ & $5.0-8|2.2-9| 1.1-10 \mid 3.9-8$ & 10 & $7.443 \mid \infty$ & | 8.71 \\
\hline qs90o & $16381 \mid ; 33124 ; 2$ & $30|-6.423774500|-6.423773610$ & $5.7-8|2.4-9| 5.5-10 \mid 6.5-8$ & 27 & $1.664 \mid \infty$ & | 1.32 \\
\hline qs 120 o & $29041 \mid ; 58564 ; 2$ & $31|-6.450146480|-6.450143990$ & $1.3-7|5.1-9| 2.5-10 \mid 1.8-7$ & 53 & $2.934 \mid \infty$ & 1.72 \\
\hline qs180o & $65161 \mid ; 131044 ; 2$ & $34|-6.483517410|-6.483511690$ & $2.0-7|7.9-9| 2.5-10 \mid 4.1-7$ & $2: 36$ & $6.554 \mid 1.33$ & $11 \mid 2.62$ \\
\hline q30o & $7482 \mid ; 11163 ; 2$ & $35|-9.36404974-1|-9.36405064-1$ & $5.5-9|5.2-10| 1.1-8 \mid 3.1-8$ & 29 & $7.443 \mid \infty$ & | 7.81 \\
\hline q60o & $29362 \mid ; 43923 ; 2$ & $44|-9.44560086-10|-2.85627290-6$ & $2.6-7|6.8-9| 7.9-9 \mid 2.9-6$ & $3: 25$ & $2.934 \mid \infty$ & 1.32 \\
\hline dsNRL & $406 \mid ; 15897 ;$ & $35|-5.57425079-5|-5.57492923-5$ & $3.3-12|1.0-12| 6.8-9 \mid 6.8-9$ & $10: 56$ & $1.054 \mid 2.10$ & $4 \mid 1.92$ \\
\hline firL1Linfa & $6224 \mid ; 35532 ;$ & $30|-2.56478569-3|-2.56479294-3$ & $1.8-11|3.3-12| 7.2-9 \mid 7.2-9$ & $17: 40$ & $1.185 \mid 2.38$ & $4 \mid 1.50$ \\
\hline firL1Linfe & $5685 \mid ; 11172 ; 1 ;$ & $42|-3.31229666-3|-3.31231116-3$ & $1.1-12|3.6-12| 1.4-8 \mid 1.4-8$ & 48 & $1.534 \mid 3.64$ & $4 \mid 5.70$ \\
\hline firL1 & $6223 \mid ; 17766 ;$ & $22|-2.92575478-4|-2.92580521-4$ & $1.1-11|1.6-12| 5.0-9 \mid 5.0-9$ & $7: 44$ & $2.414 \mid 1.18$ & $4 \mid 4.7-1$ \\
\hline firL2a & $2002 \mid ; 2003 ; ;$ & $7|-5.07008269-4|-5.07012684-4$ & $7.4-14|9.5-10| 5.9-9 \mid 4.4-9$ & $3: 00$ & $2.000 \mid 2.00$ & $0 \mid 1.40$ \\
\hline firL2L1alp & $5868 \mid ; 9611 ; 1$ & $15|-5.76343274-5|-5.76374679-5$ & $7.1-12|3.9-12| 3.1-9 \mid 3.1-9$ & 23 & $1.194 \mid 3.85$ & $3 \mid 1.80$ \\
\hline firL2L1eps & $8303 \mid ; 24108 ;$ & $23|-5.35470923-4|-5.35471575-4$ & $2.3-13|1.0-12| 6.5-10 \mid 6.5-10$ & 19:06 & $3.904 \mid 1.58$ & $4 \mid 3.1-1$ \\
\hline firL2Linfa & $303 \mid ; 13629 ;$ & $27|-6.79117295-3|-6.79117919-3$ & $5.2-12|1.2-12| 6.2-9 \mid 6.2-9$ & $4: 05$ & $1.784 \mid 8.93$ & $3 \mid 7.7-1$ \\
\hline firL2Linfe & $6086 \mid ; 14711 ;$ & $17|-1.48919871-3|-1.48920537-3$ & $9.4-11|5.2-12| 7.0-9 \mid 6.6-9$ & 1:53 & $5.893 \mid 5.83$ & $5 \mid 1.12$ \\
\hline firL2 & $102 \mid ; 103 ;$ & $7|-3.11866437-3|-3.11866351-3$ & $4.7-14|8.8-10| 4.7-10 \mid 8.6-10$ & 00 & $2.000 \mid 2.00$ & $0 \mid 1.40$ \\
\hline firLinf & $402 \mid ; 11886 ;$ & $23|-1.00681681-2|-1.00681770-2$ & $8.6-10|3.8-11| 8.7-9 \mid 8.7-9$ & $5: 20$ & $7.923 \mid 7.96$ & $3 \mid 8.1-1$ \\
\hline wbNRL & $460 \mid ; 1578 ; 17177$ & $30|-4.15006324-5|-4.15040502-5$ & $1.9-11|1.0-12| 3.4-9 \mid 3.4-9$ & $14: 14$ & $1.724 \mid 6.51$ & $5 \mid 7.30$ \\
\hline BeH-2Sigma & $948 \mid 1406 ; ; ;$ & $30|1.669356401| 1.669356391$ & $1.4-12|1.0-12| 3.6-9 \mid 3.6-9$ & $3: 29$ & $1.433 \mid 1.34$ & $10 \mid 2.41$ \\
\hline BH-1Sigma+ & $948 \mid 1406 ; ; ;$ & $30|2.720633771| 2.720633751$ & $7.0-12|1.2-12| 4.0-9 \mid 3.9-9$ & $3: 28$ & $1.443 \mid 3.71$ & $10 \mid 2.51$ \\
\hline $\mathrm{BH} 2-2 \mathrm{~A} 1-\mathrm{ST}$ & $1743 \mid 2166 ; ; ;$ & $30|3.043011671| 3.043011661$ & $3.7-10|1.6-11| 1.0-9 \mid 9.1-10$ & $15: 16$ & $2.203 \mid 4.79$ & $9 \mid 2.12$ \\
\hline $\mathrm{BH}+-2$ Sigma & $948 \mid 1406 ; ; ;$ & $29|2.697966601| 2.697966571$ & $1.6-12|1.0-12| 4.7-9 \mid 4.7-9$ & $3: 23$ & $1.443 \mid 1.34$ & $10 \mid 2.41$ \\
\hline $\mathrm{CH}+-1$ Sigma & $948 \mid 1406 ;$; ; & $29|4.069278791| 4.069278731$ & $2.4-12|1.0-12| 7.9-9 \mid 7.9-9$ & $3: 22$ & $1.453 \mid 3.71$ & $10 \mid 2.61$ \\
\hline $\mathrm{CH} 2-1 \mathrm{~A} 1-\mathrm{ST}$ & $1743 \mid 2166 ; ;$ & \begin{tabular}{l|l|l|l}
28 & 4.48537630 & 1 & 4.485376251
\end{tabular} & $4.6-10|1.3-10| 5.8-9 \mid 5.2-9$ & $14: 17$ & $2.213 \mid 4.95$ & $9 \mid 6.21$ \\
\hline
\end{tabular}


Table 1: Performance of sdpt3.m. In the table, err = [pinfeas, dinfeas, relgap, relgap2], where relgap2 is the same as relgap but with the numerator replaced by $\left|\langle c, x\rangle-b^{T} y\right|$, and normXZ $=\max \left\{\left\|x^{*}\right\|,\left\|z^{*}\right\|\right\}$. We declare that $g_{P}\left(g_{D}\right)$ is $\infty$ if the computed number is larger than $10^{12}$

\begin{tabular}{|c|c|c|c|c|c|c|}
\hline problem & $m \mid n_{s} ; n_{q} ; n_{l} ; n_{u}$ & & it. | primal obj | dual obj & err & time & $g_{P}\left|g_{D}\right|$ normXZ \\
\hline $\mathrm{CH} 2-3 \mathrm{~B} 1-\mathrm{ST}$ & $1743 \mid 2166 ; ; ;$ & $29 \mid$ & $4.502913291 \mid 4.502913271$ & $2.8-10|3.8-11| 1.7-9 \mid 1.5-9$ & $14: 45$ & $2.223|4.739| 1.32$ \\
\hline $\mathrm{CH}-2 \mathrm{Pi}-\mathrm{STO}$ & $948 \mid 1406 ;$; ; & $28 \mid$ & $4.102221781 \mid 4.102221761$ & $2.6-11|4.4-12| 2.3-9 \mid 2.2-9$ & $3: 18$ & \begin{tabular}{ll|ll|l}
1.45 & 3 & 1.59 & $10 \mid$ & 4.41
\end{tabular} \\
\hline $\mathrm{CH}-3$ Sigma & $948 \mid 1406 ; ; ;$ & $28 \mid$ & $4.090709131 \mid 4.090709091$ & $8.4-12|1.5-12| 5.5-9 \mid 5.5-9$ & $3: 16$ & \begin{tabular}{ll|ll|l}
1.45 & 3 & 1.66 & $10 \mid$ & 3.21
\end{tabular} \\
\hline $\mathrm{H} 2 \mathrm{O}-1 \mathrm{~A} 1-\mathrm{ST}$ & $1743 \mid 2166 ; ; ;$ & 28 & 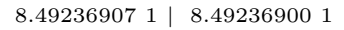 & $3.7-12|2.0-12| 3.9-9 \mid 3.9-9$ & $14: 15$ & \begin{tabular}{lll|l|l|}
2.24 & 3 & 6.09 & 9 & 4.41
\end{tabular} \\
\hline $\mathrm{H} 2 \mathrm{O}+-2 \mathrm{~B} 1-\mathrm{S}$ & $1743 \mid 2166 ; ; ;$ & $29 \mid$ & $8.421637641 \mid 8.421637591$ & $4.6-11|1.1-11| 2.8-9 \mid 2.8-9$ & $14: 47$ & $2.243|5.499| 6.71$ \\
\hline HF-1Sigma+ & $948 \mid 1406 ;$; ; & $27 \mid$ & $1.047204542 \mid 1.047204522$ & $2.5-12|1.0-12| 8.7-9 \mid 8.7-9$ & $3: 08$ & $1.493|1.749| 3.71$ \\
\hline $\mathrm{HF}+-2 \mathrm{Pi}-\mathrm{ST}$ & $948 \mid 1406 ; ; ;$ & $26 \mid$ & $1.038856682 \mid 1.038856662$ & $3.2-11|1.0-12| 9.4-9 \mid 9.5-9$ & $3: 01$ & \begin{tabular}{ll|ll|ll}
1.49 & 3 & 1.75 & $10 \mid$ & 3.2 & 1
\end{tabular} \\
\hline LiH-1Sigma & $948 \mid 1406 ; ; ;$ & 29 | & 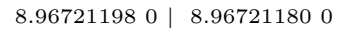 & $9.1-12|2.5-12| 9.2-9 \mid 9.2-9$ & $3: 23$ & 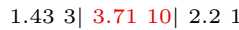 \\
\hline NH2-2B1-ST & $1743 \mid 2166 ; ; ;$ & $29 \mid$ & $6.297980181 \mid 6.297980151$ & $7.5-11|1.7-11| 2.7-9 \mid 2.7-9$ & $14: 49$ & $2.233|5.499| 6.81$ \\
\hline $\mathrm{NH}+-2 \mathrm{Pi}-\mathrm{ST}$ & $948 \mid 1406 ;$; ; & 28 & \begin{tabular}{ll|l}
5.78593622 & 1 & 5.785936191
\end{tabular} & 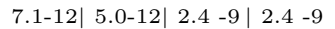 & $3: 16$ & \begin{tabular}{ll|ll|l}
1.46 & 3 & 1.59 & $10 \mid$ & 4.01
\end{tabular} \\
\hline $\mathrm{NH}-2 \mathrm{Pi}-\mathrm{ST}$ & $948 \mid 1406 ; ; ;$ & $28 \mid$ & \begin{tabular}{ll|l}
5.805463961 & 5.805463881
\end{tabular} & $9.7-11|1.0-12| 6.9-9 \mid 6.9-9$ & $3: 15$ & 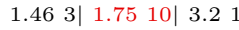 \\
\hline NH-3Sigma- & $948 \mid 1406 ; ;$ & $27 \mid$ & $5.839100251 \mid 5.839100161$ & $3.4-11|1.6-12| 7.7-9 \mid 7.7-9$ & $3: 08$ & \begin{tabular}{ll|ll|ll}
1.46 & 3 & 1.66 & $10 \mid$ & 3.2 & 1
\end{tabular} \\
\hline OH-1Sigma & $948 \mid 1406 ;$; ; & $29 \mid$ & $7.916806021 \mid 7.916805861$ & $1.5-12|1.5-12| 1.0-8 \mid 1.0-8$ & $3: 23$ & \begin{tabular}{ll|l|l|l}
1.48 & 3 & 1.74 & 9 & 3.7
\end{tabular} \\
\hline $\mathrm{OH}-2 \mathrm{Pi}-\mathrm{STO}$ & $948 \mid 1406 ; ; ;$ & $27 \mid$ & $7.946707711 \mid 7.946707631$ & $2.3-12|1.0-12| 5.4-9 \mid 5.4-9$ & $3: 10$ & \begin{tabular}{ll|ll|ll}
1.48 & 3 & 1.75 & $10 \mid$ & 3.2 & 1
\end{tabular} \\
\hline $\mathrm{OH}+-3 \mathrm{Sigma}$ & $948 \mid 1406 ; ; ;$ & $27 \mid$ & \begin{tabular}{ll|ll}
7.88863798 & 1 & 7.88863789 & 1
\end{tabular} & $1.8-11|1.5-12| 5.6-9 \mid 5.6-9$ & $3: 09$ & \begin{tabular}{ll|ll|ll}
1.48 & 3 & 1.66 & $10 \mid$ & 3.2 & 1
\end{tabular} \\
\hline Li.2S.STO6 & $465 \mid 780 ; ; ; 35$ & 351 & $\begin{array}{lll}7.40023852 & 0 & 7.400238280\end{array}$ & 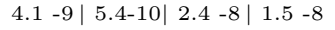 & 46 & $7.862 \mid \infty$ \\
\hline Be.1S.STO6 & $465 \mid 780 ; ; ; 35$ & $38 \mid$ & $1.455608981 \mid 1.455608851$ & $4.7-8|7.9-10| \begin{array}{llll}4 & 4 & -8 & -8\end{array}$ & 53 & $7.872 \mid \infty$ \\
\hline $\mathrm{BeH}+.1$ Sigm & $948 \mid 1312 ; ; ; 47$ & $40 \mid$ & $1.645750961 \mid 1.645750711$ & $8.3-8|8.4-10| 1.0-7 \mid 7.4-8$ & $4: 17$ & $1.323 \mid \infty$ \\
\hline $\mathrm{H} 3.2 \mathrm{~A} 1 . \mathrm{DZ}$. & $948 \mid 1312 ; ; ; 47$ & $38 \mid$ & $3.364654330 \mid 3.364647990$ & $5.2-8 \mid \begin{array}{llllllll}5 & 5.1 & -9 & 1.6 & -6 & 8.2 & -7\end{array}$ & $3: 59$ & $1.323 \mid \infty$ \\
\hline $\mathrm{FH} 2+.1 \mathrm{~A} 1 . \mathrm{S}$ & $1743 \mid 2044 ; ; ; 61$ & $42 \mid$ & \begin{tabular}{ll|l}
1.099904092 & 1.099904012
\end{tabular} & $3.1-8|3.5-10| 5.1-8 \mid 3.4-8$ & $20: 37$ & $2.063 \mid \infty$ \\
\hline NH2-.1A $1 . \mathrm{S}$ & $1743 \mid 2044 ; ; ; 61$ & $41 \mid$ & $6.270621741 \mid 6.270621391$ & $2.7-8|2.9-10| 5.0-8 \mid 2.7-8$ & $20: 15$ & $2.063 \mid \infty$ \\
\hline quadknap-1 & $5984 \mid 189 ; ; 5814$ & $45 \mid$ & $1.204672773 \mid 1.204672773$ & $1.6-8|1.2-13| 2.1-10 \mid 1.6-10$ & $9: 21$ & $1.614 \mid \infty$ \\
\hline quadknap-1 & $5984 \mid 189 ; ; 5814$ & 35 & $\begin{array}{l}4.927359123 \mid 4.927368063\end{array}$ & $8.9-7|4.8-8| 3.5-6 \mid 9.1-7$ & $7: 26$ & $1.594 \mid \infty$ \\
\hline quadknap-1 & $5984 \mid 189 ; ; 5814$ & $37 \mid$ & $4.848814983 \mid 4.848823473$ & $9.5-7|3.8-8| 2.5-6 \mid 8.8-7$ & $11: 18$ & $1.614 \mid \infty$ \\
\hline quadknap-1 & $5984 \mid 189 ; ; 5814$ & $44 \mid$ & $7.885015072 \mid 7.885015062$ & $7.7-8|1.9-13| 5.7-10 \mid 3.1-10$ & $12: 07$ & $1.704 \mid \infty$ \\
\hline quadknap-1 & $5984 \mid 189 ; ; 5814$ & $39 \mid$ & $5.375147863 \mid 5.375155993$ & $7.9-7|3.8-8| \begin{array}{lllll} & 1.6 & -6 & 7.6 & -7\end{array}$ & $11: 56$ & $1.704 \mid \infty$ \\
\hline quadknap-1 & $5984 \mid 189 ; ; 5814$ & 39 & \begin{tabular}{ll|l|l}
2.40839711 & 3 & 2.408397583
\end{tabular} & $2.5-7|1.3-8| 3.3-7 \mid 9.8-8$ & $8: 13$ & $1.514 \mid \infty$ \\
\hline quadknap-1 & $5984 \mid 189 ; ; 5814$ & $39 \mid$ & $8.048000023 \mid 8.048000003$ & $5.4-8|1.2-11| 4.3-10 \mid 9.6-10$ & $8: 17$ & $1.874 \mid \infty$ \\
\hline quadknap-1 & $5984 \mid 189 ; ; 5814$ & $38 \mid$ & $5.851000083 \mid 5.851000003$ & $2.9-8|5.8-11| 7.7-10 \mid 6.5-9$ & $8: 02$ & $1.584 \mid \infty$ \\
\hline quadknap-1 & $5984 \mid 189 ; ; 5814$ & $37 \mid$ & $\begin{array}{l}5.132591903 \mid \\
\end{array}$ & $2.0-7|6.2-8| 3.7-7 \mid 3.8-7$ & $7: 46$ & $1.634 \mid \infty$ \\
\hline quadknap-1 & $5984 \mid 189 ; ; 5814$ & $40 \mid$ & $6.775000033 \mid 6.775000003$ & $1.5-6|3.6-11| 9.6-10 \mid 1.8-9$ & $8: 22$ & $1.944 \mid \infty$ \\
\hline stable-17- & $5984 \mid 477 ; ; 342$ & 29 & $-1.42857143-1 \mid-1.42857142-1$ & $3.6-10|6.2-10| 1.3-9 \mid 3.2-10$ & $6: 20$ & $\infty \quad \mid \infty$ \\
\hline stable-17- & $5984 \mid 477 ; ; 342$ & $30 \mid$ & $-1.98434223-1 \mid-1.98434223-1$ & $7.5-11|1.9-11| 3.4-10 \mid 7.7-11$ & $6: 33$ & 1.12 \\
\hline stable-17- & $5984 \mid 477 ; ; 342$ & $28 \mid$ & $-1.66666666-1 \mid-1.666666666-1$ & $2.6-10|7.2-10| 1.2-9 \mid 6.5-10$ & $6: 13$ & 6.81 \\
\hline stable-17- & $5984 \mid 477 ; ; 342$ & $36 \mid$ & $-1.66131915-1 \mid-1.66131921-1$ & $4.2-9|2.8-11| 9.9-9 \mid 3.9-9$ & $7: 48$ & 1.62 \\
\hline stable-17- & $5984 \mid 477 ; ; 342$ & $34 \mid$ & $-1.95961595-1 \mid-1.95961595-1$ & $7.6-10|4.7-12| 6.1-10 \mid 1.2-12$ & $7: 15$ & 1.62 \\
\hline stable-17- & $5984 \mid 477 ; ; 342$ & $36 \mid$ & $-1.95658388-1 \mid-1.95658390-1$ & $7.8-10|1.9-11| 3.8-9 \mid 1.4-9$ & $7: 36$ & 1.82 \\
\hline stable-17- & $5984 \mid 477 ; ; 342$ & $39 \mid$ & $-1.66561011-1 \mid-1.66561013-1$ & $2.8-8|6.6-13| 5.1-10 \mid 1.3-9$ & $9: 03$ & 1.72 \\
\hline stable-17- & $5984 \mid 477 ; ; 342$ & $28 \mid$ & $-1.66666667-1 \mid-1.666666666-1$ & $1.2-10|3.9-10| 5.3-10 \mid 4.0-10$ & $6: 04$ & | 6.61 \\
\hline stable-17- & $5984 \mid 477 ; ; 342$ & $32 \mid$ & $-1.66138271-1 \mid-1.66138271-1$ & $9.8-11|1.6-11| 6.8-10 \mid 2.5-10$ & $7: 01$ & | 1.42 \\
\hline stable-17- & $5984 \mid 477 ; ; 342$ & $29 \mid$ & $-1.66138270-1 \mid-1.66138270-1$ & $6.6-10|8.8-10| 4.9-9 \mid 3.5-10$ & $6: 05$ & 9.91 \\
\hline MaxCut-100 & $6252 \mid 1850 ; ; 4188$ & $35 \mid$ & $1.460726202 \mid 1.460679652$ & $1.2-5 \mid$\begin{tabular}{ll|ll|ll} 
& 2.9 & -6 & 8.7 & -5 & -5
\end{tabular} & 50 & $9.593 \mid \infty$ \\
\hline MaxCut-100 & $6252 \mid 1850 ; ; 4188$ & $35 \mid$ & $1.460726202 \mid 1.460679652$ & $1.2-5|2.9-6| 8.7-5 \mid 1.6-5$ & 50 & $9.593 \mid \infty$ \\
\hline MaxCut-100 & $7767 \mid 2134 ; ; 4590$ & $44 \mid$ & \begin{tabular}{ll|l}
1.480434352 & 1.480434392
\end{tabular} & $5.8-6|3.9-10| 3.7-9 \mid 1.2-8$ & $3: 00$ & $1.094 \mid \infty$ \\
\hline MaxCut-100 & $5679 \mid 1775 ; ; 3876$ & 36 & \begin{tabular}{l|l}
$1.470650952 \mid$ & 1.470637932
\end{tabular} & $1.4-5|2.8-6| 3.4-5 \mid 4.4-6$ & 42 & $9.033 \mid \infty$ \\
\hline MaxCut-100 & $6717 \mid 1877 ; ; 4476$ & $36 \mid$ & $1.340599302 \mid 1.340570812$ & 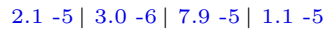 & $1: 46$ & $9.953 \mid \infty$ \\
\hline MaxCut-100 & 6059 | 1759; ; 4044; & $34 \mid$ & $1.470284562 \mid 1.470284782$ & 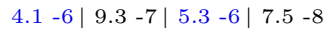 & $1: 21$ & $9.193 \mid \infty$ \\
\hline MaxCut-100 & $7221 \mid 2103 ; ; 4900$ & $35 \mid$ & $1.480382762 \mid 1.480360862$ & $2.0-5 \mid$\begin{tabular}{ll|ll|ll} 
& 4.3 & -6 & 7.2 & -5 & -6
\end{tabular} & $1: 59$ & $1.104 \mid \infty$ \\
\hline MaxCut-100 & $7375 \mid 2121 ; ; 4400$ & $35 \mid$ & $1.470512362 \mid 1.470512412$ & $1.7-6 \mid$\begin{tabular}{ll|ll|ll} 
& 3.2 & -7 & -6 & -6 & -6
\end{tabular} & $1: 22$ & $1.064 \mid \infty$ \\
\hline MaxCut-100 & $6495 \mid 1937 ; ; 4328$ & $35 \mid$ & \begin{tabular}{ll|l}
1.340967962 & 1.340947922
\end{tabular} & 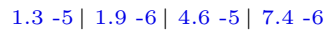 & $1: 37$ & $9.953 \mid \infty$ \\
\hline MaxCut-100 & $7228 \mid 1923 ; ; 4816$ & $35 \mid$ & $1.450155922 \mid 1.450119272$ & 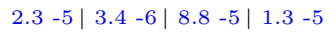 & $2: 04$ & $1.054 \mid \infty$ \\
\hline Bex2-1-1.g & $251 \mid 252 ; ; 182$ & $22 \mid$ & 3.40000004-1 | 3.39999990-1 & $5.1-10|1.9-11| 9.6-9 \mid 8.5-9$ & 02 & $6.212|9.144| 3.31$ \\
\hline Bex2-1-2.g & $65 \mid 80 ; ; 52$ & $17 \mid$ & $1.065000000 \mid 1.065000000$ & $4.9-13|1.0-12| 5.9-10 \mid 5.8-10$ & 00 & $1.752|3.343| 1.01$ \\
\hline Bex2-1-3.g & $134 \mid 193 ;$; 113 ; & $18 \mid$ & $1.875000000 \mid 1.875000000$ & $4.0-13|1.0-12| 4.9-10 \mid 4.8-10$ & 01 & \begin{tabular}{ll|ll|ll}
4.44 & 2 & 7.70 & $4 \mid 2.81$
\end{tabular} \\
\hline Bex2-1-4.g & $83 \mid 126 ; ; 61$ & $17 \mid$ & $1.375000010 \mid 1.374999980$ & $1.6-12|1.0-12| 7.8-9 \mid 7.8-9$ & 00 & 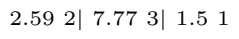 \\
\hline Bex2-1-5.g & $285 \mid 352 ; ; 295$ & $21 \mid$ & $2.977940360 \mid 2.977940340$ & $1.1-9|1.2-11| 3.2-9 \mid 3.1-9$ & 02 & \begin{tabular}{ll|ll|ll}
9.80 & 2 & 5.30 & 5 & 7.6 & 1
\end{tabular} \\
\hline Bex2-1-8.g & $1789 \mid 798 ; ; 2655 ; 596$ & $37 \mid$ & $-7.85090352-1 \mid-7.85090364-1$ & $5.4-10|1.3-9| 9.0-9 \mid 4.7-9$ & 25 & $5.103 \mid \infty$ \\
\hline Bex3-1-1.g & $310 \mid 300 ; ; 604$ & $24 \mid$ & 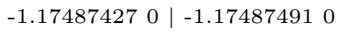 & $2.9-7|9.7-12| 2.5-7 \mid 1.9-7$ & 02 & $1.093|2.1310| 4.33$ \\
\hline Bex3-1-2.g & 111 | 110; ; 212; & $19 \mid$ & $-9.33357008-1 \mid-9.33357019-1$ & $1.5-10|8.1-12| 4.3-9 \mid 3.9-9$ & 01 & $3.602|4.545| 5.81$ \\
\hline Bex3-1-4.g & $164 \mid 215 ; ; 73$ & $21 \mid$ & $\begin{array}{llll}1.00009958 & 0 & 1.000105210\end{array}$ & $1.2-5|3.9-10| 2.6-6 \mid 1.9-6$ & 03 & \begin{tabular}{ll|ll|ll}
4.52 & 2 & 1.42 & 6 & 2.23
\end{tabular} \\
\hline Bex5-2-2-c & $300 \mid 212 ; ; 359 ; 220$ & $40 \mid$ & 8.33333333-2 | 8.33333333-2 & $1.1-8|4.1-15| 5.0-13 \mid 1.5-13$ & 02 & $1.153 \mid \infty$ \\
\hline Bex5-2-2-c & $300 \mid 212 ; ; 359 ; 220$ & 36 & $\begin{array}{l}7.50000087-2 \mid 7.50000039-2\end{array}$ & 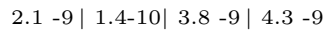 & 02 & $1.153 \mid \infty$ \\
\hline
\end{tabular}


Table 1: Performance of sdpt3.m. In the table, err = [pinfeas, dinfeas, relgap, relgap2], where relgap2 is the same as relgap but with the numerator replaced by $\left|\langle c, x\rangle-b^{T} y\right|$, and normXZ $=\max \left\{\left\|x^{*}\right\|,\left\|z^{*}\right\|\right\}$. We declare that $g_{P}\left(g_{D}\right)$ is $\infty$ if the computed number is larger than $10^{12}$

\begin{tabular}{|c|c|c|c|c|c|}
\hline problem & $m \mid n_{s} ; n_{q} ; n_{l} ; n_{u}$ & it. | primal obj | dual obj & err & time & $g_{P}\left|g_{D}\right|$ normXZ \\
\hline Bex5-2-2-c & $300 \mid 212 ; ; 359 ; 220$ & $27|1.92307719-1| 1.92307733-1$ & 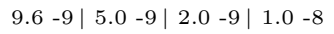 & 02 & $1.153 \mid \infty$ \\
\hline Bex5-3-2.g & $1131 \mid 495 ; ; 1637 ; 530$ & $46|-1.30519579-1|-1.30523903-1$ & 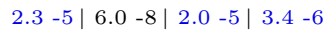 & 06 & $3.603 \mid \infty$ \\
\hline Bex5-4-2.g & $310 \mid 300 ; ; 604$ & $28|-7.51223010-1|-7.51223017-1$ & $4.2-8|6.2-14| 2.9-9 \mid 2.7-9$ & 02 & $1.093|2.028| 4.23$ \\
\hline Bex9-1-1.g & $489 \mid 368 ; ; 548 ; 699$ & $29|2.16666669-1| 2.16666667-1$ & $1.0-9|8.9-9| 3.7-9 \mid 1.0-9$ & 04 & $2.533 \mid \infty$ \\
\hline Bex9-1-2.g & $241 \mid 223 ; ; 227 ; 310$ & $28|1.066666670| 1.066666670$ & $2.7-9|1.6-10| 5.1-11 \mid 2.3-9$ & 01 & \begin{tabular}{llll|ll}
1.20 & 3 & 1.59 & $10 \mid$ & 1.5 & 1
\end{tabular} \\
\hline Bex9-1-4.g & $241 \mid 223 ; ; 227 ; 310$ & $24|6.60872427-1| 6.60872425-1$ & $6.5-10|2.6-9| 4.4-10 \mid 6.6-10$ & 02 & $1.203 \mid \infty$ \\
\hline Bex9-1-5.g & $489 \mid 368 ; ; 548 ; 700$ & $28|2.01411716-2| 2.01411716-2$ & $3.6-11|2.6-10| 4.4-11 \mid 3.2-11$ & 04 & $2.523 \mid \infty$ \\
\hline Bex9-1-8.g & $402 \mid 330 ; ; 434 ; 522$ & $29|6.50000034-1| 6.50000005-1$ & $1.3-8|1.9-8| 6.5-9 \mid 1.2-8$ & 03 & $2.013|4.42 \quad 10| 1.71$ \\
\hline Bex9-2-1.g & $241 \mid 223 ; ; 227 ; 310$ & \begin{tabular}{l|l|l}
25 & $4.59183756-2 \mid$ & $4.59183697-2$
\end{tabular} & $4.4-9|5.8-9|$\begin{tabular}{lll|l}
5 & 1.9 & -9 & 5.4
\end{tabular} & 02 & $1.183 \mid \infty$ \\
\hline Bex9-2-2.g & $137 \mid 156 ; ; 109 ; 168$ & \begin{tabular}{l|l|l|}
42 & $2.87494906-6$ & $25999495-6$
\end{tabular} & 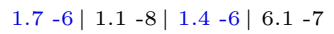 & 02 & $6.862|1.0811| 5.12$ \\
\hline Bex9-2-3.g & $866 \mid 549 ; ; 1408 ; 1176$ & $35|-1.199999940|-1.200000000$ & $1.4-10|5.8-10| 1.7-9 \mid 1.7-8$ & 11 & $6.153|3.31 \quad 10| 4.42$ \\
\hline Bex9-2-4.g & $146 \mid 149 ; ; 276 ; 215$ & $26|1.75001038-4| 1.75000009-4$ & $2.9-10|6.5-10| 2.6-9 \mid 1.0-9$ & 02 & $9.092 \mid \infty$ \\
\hline Bex9-2-5.g & $137 \mid 147 ; ; 109 ; 168$ & $24|8.00000007-2| 8.00000001-2$ & $5.1-10|1.8-10| 2.1-11 \mid 5.7-10$ & 01 & $6.662 \mid \infty$ \\
\hline Bex9-2-6.g & $402 \mid 317 ; ; 434 ; 522$ & $28|2.42789755-5| 2.42661365-5$ & $6.9-9 \mid$\begin{tabular}{ll|ll|ll} 
& 1.5 & -9 & 1.9 & -9 & -8
\end{tabular} & 03 & $1.963 \mid \infty$ \\
\hline Bex9-2-7.g & $241 \mid 223 ; ; 227 ; 310$ & $26|4.59183796-2| 4.59183677-2$ & $7.6-9 \mid$\begin{tabular}{ll|ll|l} 
& 2.5 & -9 & 1.3 & -9 \\
& 1.1 & -8
\end{tabular} & 01 & \begin{tabular}{ll|lll}
1.18 & 3 & 1.57 & $10 \mid$ & 1.31
\end{tabular} \\
\hline Bex9-2-8.g & $19 \mid 28 ; ; 8 ; 50$ & $16 \mid$\begin{tabular}{l|l}
$1.70246919-11 \mid 3.26220519-10$
\end{tabular} & $2.9-12|6.3-9| 1.2-10 \mid 3.1-10$ & 00 & $1.462|\infty \quad| 2.80$ \\
\hline Balkyl.gms & $834 \mid 572 ; ; 1640 ; 310$ & $32|5.89807109-2| 5.89807733-2$ & $2.7-9|4.3-10| 4.4-9 \mid 5.6-8$ & 05 & \begin{tabular}{llll|ll}
3.09 & 3 & 7.12 & $10 \mid$ & 4.9 & 1
\end{tabular} \\
\hline Bst-bpaf1a & $195 \mid 245 ; ; 205$ & $31|1.13449275-1| 1.13449275-1$ & $1.2-10|1.1-14| 3.9-10 \mid 3.4-10$ & 02 & $6.072|3.648| 2.23$ \\
\hline Bst-bpaf1b & $195 \mid 245 ;$; 205; & $28|1.07406396-1| 1.07406388-1$ & $2.7-10|1.3-13| 7.4-9 \mid 6.5-9$ & 01 & $6.082|3.648| 4.13$ \\
\hline Bst-e05.gm & $40 \mid 44 ; ; 70 ; 22$ & $23|-1.94462048-1|-1.94462044-1$ & $8.7-10|7.0-10| 2.4-10 \mid 3.4-9$ & 00 & \begin{tabular}{ll|l}
$1.772 \mid \infty$ & 2.11
\end{tabular} \\
\hline Bst-e07.gm & $178 \mid 161 ; ; 189 ; 82$ & $25|3.76913266-1| 3.76913266-1$ & $1.6-10|2.7-9| 5.4-10 \mid 1.8-10$ & 01 & $6.452 \mid \infty$ \\
\hline Bst-jcbpaf & $285 \mid 374 ; ; 295$ & $25|7.94855924-2| 7.94855883-2$ & $1.1-11|2.0-12| 3.6-9 \mid 3.5-9$ & 02 & $8.762|6.545| 1.71$ \\
\hline Bhaverly.g & $274 \mid 206 ; ; 331 ; 154$ & \begin{tabular}{l|l|l}
46 & $8.00000000-2$ & $8.00000000-2$
\end{tabular} & $2.4-7|1.6-14| 5.5-12 \mid 1.6-13$ & 02 & $9.822 \mid \infty$ \\
\hline alkylation & $530 \mid 457 ; ; 1040 ; 155$ & $44|1.18826559-2| 1.18824854-2$ & $3.1-7|7.0-11| 6.7-9 \mid 1.7-7$ & 06 & $2.003|5.95 \quad 10| 2.82$ \\
\hline Bst-bpk1.g & $34 \mid 55 ; ; 30$ & $14|1.300000001| 1.300000001$ & $7.5-13|1.0-12| 1.6-9 \mid 1.6-9$ & 00 & $7.012|3.282| 1.62$ \\
\hline Bst-bpk2.g & $34 \mid 55 ; ; 30$ & $14|1.300000001| 1.300000001$ & $7.5-13|1.0-12| 1.6-9 \mid 1.6-9$ & 00 & \begin{tabular}{ll|l|l}
7.012 & 2 & 3.28 & 2 \\
1.62
\end{tabular} \\
\hline Bst-bpv1.g & $29 \mid 56 ; ; 17$ & $13|-3.70370361-2|-3.70370407-2$ & $4.6-10|1.7-12| 6.7-9 \mid 4.3-9$ & 00 & \begin{tabular}{ll|l}
$1.012 \mid \infty$ & 5.72
\end{tabular} \\
\hline Bst-bpv2.g & $25 \mid 52 ; ; 14$ & $14|4.00000003-1| 3.99999993-1$ & $1.1-13|1.0-12| 5.7-9 \mid 5.7-9$ & 00 & $8.661|2.28 \quad 3| 5.90$ \\
\hline Bst-e42.gm & $104 \mid 78 ; ; 103 ; 56$ & $33|-1.878419991|-1.878420001$ & $1.3-8|1.8-9| 5.4-9 \mid 2.3-9$ & 01 & \begin{tabular}{l|l|l}
$\infty$ & $\infty$ & 9.83
\end{tabular} \\
\hline Bst-robot. & $494 \mid 189 ; ; 972 ; 360$ & $23|2.25821389-4| 2.25821334-4$ & $1.3-12|6.5-10| 8.1-11 \mid 5.4-11$ & 02 & $1.983|1.6311| 4.31$ \\
\hline Bprolog.gm & $833 \mid 533 ; ; 1023$ & $91|5.22890377-4|-2.14756935-6$ & $9.8-13|1.6-7| 6.7-4 \mid 5.2-4$ & 14 & | $3.955 \mid 3.310$ \\
\hline st-cqpjk2. & $19 \mid 32 ; ; 8$ & $13|8.33333343-1| 8.33333317-1$ & $1.2-11|1.0-12| 9.9-9 \mid 9.9-9$ & 00 & $5.611 \mid \begin{array}{lllll}5.32 & 2 & 4.9 & 0\end{array}$ \\
\hline st-e01.gms & $12 \mid 19 ; ; 8$ & $18|1.111111130| 1.111111120$ & $4.9-13|1.0-12| 4.0-9 \mid 4.0-9$ & 00 & $3.501|6.332| 3.90$ \\
\hline st-e09.gms & $25 \mid 38 ; ; 12$ & $14|2.50000000-1| 2.50000000-1$ & $1.8-11|1.0-12| 3.9-10 \mid 3.5-10$ & 00 & $6.761|2.304| 2.61$ \\
\hline st-e10.gms & $10 \mid 16 ; ; 3 ; 1$ & $17|6.97453884-1| 6.97453928-1$ & $2.0-9|5.7-9| 4.7-10 \mid 1.8-8$ & 00 & $2.941 \mid \infty$ \\
\hline st-e20.gms & $111 \mid 84 ; ; 210 ; 72$ & $23|2.67968679-1| 2.67968687-1$ & $1.6-9|2.9-9| 6.9-10 \mid 4.9-9$ & 01 & $4.842 \mid \infty$ \\
\hline st-e23.gms & $9 \mid 21 ; ; 3 ;$ & $14|4.33333353-2| 4.33333298-2$ & $2.5-11|1.5-12| 5.1-9 \mid 5.1-9$ & 00 & $2.871|2.904| 1.01$ \\
\hline st-e34.gms & $164 \mid 125 ; ; 187$ & $20|-3.62873428-3|-3.62873545-3$ & $2.6-14|1.0-12| 1.2-9 \mid 1.2-9$ & 01 & $4.432|4.573| 9.30$ \\
\hline st-e42.gms & $104 \mid 78 ; ; 103 ; 56$ & $33|-1.878419991|-1.878420001$ & $1.3-8|1.8-9| 5.4-9 \mid 2.3-9$ & 02 & 9.83 \\
\hline st-fp5.gms & $285 \mid 352 ; ; 295$ & $21|2.977940360| 2.977940340$ & $1.1-9|1.2-11| 3.2-9 \mid 3.1-9$ & 02 & $9.802|5.305| 7.61$ \\
\hline st-glmp-fp & $34 \mid 55 ; ; 14 ; 30$ & $19|-9.999999940|-9.999999920$ & $1.7-9|5.5-9| 6.6-10 \mid 9.9-10$ & 00 & $6.942 \mid \infty$ \\
\hline st-glmp-fp & $125 \mid 180 ; ; 36 ; 140$ & $29|-7.344545410|-7.344545420$ & $1.0-10|3.7-10| 4.2-10 \mid 3.8-10$ & 02 & $1.664 \mid \infty$ \\
\hline st-glmp-fp & $34 \mid 55 ; ; 14 ; 30$ & $20|1.200000001| 1.200000001$ & $1.4-9|3.2-10| 1.4-11 \mid 1.6-10$ & 00 & $1.093 \mid \infty$ \\
\hline st-glmp-kk & $40 \mid 44 ; ; 14 ; 36$ & $20|-3.000000000|-2.999999990$ & $4.9-10|7.4-10| 2.7-11 \mid 1.4-9$ & 01 & $6.172 \mid \infty$ \\
\hline st-glmp-kk & $34 \mid 55 ; ; 14 ; 30$ & \begin{tabular}{l|l|l|l|l}
20 & 1.20000000 & 1 & 1.20000000 & 1
\end{tabular} & $2.3-9|2.2-9| 1.7-10 \mid 6.4-10$ & 00 & $7.312 \mid \infty$ \\
\hline st-glmp-kk & $69 \mid 62 ; ; 14 ; 70$ & $29|2.500000030| 2.500000050$ & $2.4-9|1.8-10| 4.6-9 \mid 2.6-9$ & 01 & $8.303 \mid \infty$ \\
\hline st-glmp-ss & $40 \mid 64 ; ; 14 ; 36$ & \begin{tabular}{l|l|l|l}
21 & 2.45714286 & 1 & 2.457142861
\end{tabular} & $1.6-9|7.2-10| 7.0-11 \mid 1.0-10$ & 00 & $4.063 \mid \infty$ \\
\hline st-glmp-ss & $40 \mid 49 ; ; 14 ; 36$ & $20|-2.999999960|-2.999999960$ & $5.8-9|1.9-9| 1.7-10 \mid 2.6-10$ & 00 & $1.283 \mid \infty$ \\
\hline st-iqpbk 1 . & $164 \mid 216 ; ; 312$ & \begin{tabular}{l|l|l|l} 
& 4.15321990 & 0 & 4.153219890
\end{tabular} & $4.5-13|1.0-12| 6.2-10 \mid 6.1-10$ & 01 & $6.942|1.764| 5.41$ \\
\hline st-iqpbk2. & $164 \mid 216 ; ; 312$ & $19|3.993670310| 3.993670310$ & $3.4-13|1.0-12| 7.3-10 \mid 7.3-10$ & 01 & $6.942|1.764| 5.41$ \\
\hline st-jcbpaf2 & $285 \mid 374 ; ; 295$ & $25|7.94855924-2| 7.94855883-2$ & $1.1-11|2.0-12| 3.6-9 \mid 3.5-9$ & 02 & $8.762|6.545| 1.71$ \\
\hline st-jcbpafe & $9 \mid 21 ; ; 3 ;$ & $14|4.33333353-2| 4.33333298-2$ & $2.5-11|1.5-12| 5.1-9 \mid 5.1-9$ & 00 & 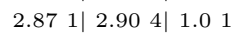 \\
\hline qp5.gms & $108 \mid ; ; 109 ; 30$ & $23|-4.31455897-1|-4.31455880-1$ & $1.5-9|9.2-10| 6.3-10 \mid 9.5-9$ & 00 & $\begin{array}{lll}1.772 \mid \infty & 4.01\end{array}$ \\
\hline Rosenbrock & $1988 \mid 1195 ; ; 3 ;$ & $18|9.95000004-1| 9.94999999-1$ & $1.4-10|1.4-12| 5.8-10 \mid 1.8-9$ & 01 & $1.285|2.353| 8.51$ \\
\hline BroydenBan & $923 \mid 84 ; ; ;$ & $17|2.40000000-1| 2.39999999-1$ & $7.4-12|1.0-12| 4.9-10 \mid 4.9-10$ & 06 & \begin{tabular}{ll|ll|ll}
5.16 & 2 & 1.54 & 2 & 5.7 & 0
\end{tabular} \\
\hline BroydenTri & $3974 \mid 1984 ; ; 3$ & $16 \mid$\begin{tabular}{l|l|l}
1.11111111 & 1 & 1.111111111
\end{tabular} & $4.3-12|3.3-12| 4.8-10 \mid 4.8-10$ & 03 & $1.754|3.343| 6.11$ \\
\hline ChainedSin & $3974 \mid 1980 ;$ & $24|2.88480646-10|-2.56585137-9$ & $4.5-14|1.0-12| 2.9-9 \mid 2.9-9$ & 03 & $2.927|2.773| 1.41$ \\
\hline ChainedWoo & $899 \mid 697 ; ; ;$ & $11|1.094210531| 1.094210521$ & $3.5-12|1.0-12| 6.4-9 \mid 6.4-9$ & 00 & $4.435|9.792| 5.01$ \\
\hline nondquar(2 & $3974 \mid 1980 ;$ & $24|2.34095452-6|-1.86415946-6$ & $2.5-9|2.2-10| 8.1-6 \mid 4.2-6$ & 04 & | $2.773 \mid 1.15$ \\
\hline $\operatorname{nonscomp}(8$ & $110 \mid 138 ;$; 204; & $23|1.56250002-2| 1.56249998-2$ & $1.4-9|7.6-12| 7.5-10 \mid 3.9-10$ & 01 & $4.472 \mid \begin{array}{lllll}5.12 & 3 & 1.6 & 1\end{array}$ \\
\hline optControl & $2682 \mid 533 ; ; ; 682$ & $25|3.30330643-1| 3.30330643-1$ & $5.3-11|3.1-10| 4.2-10 \mid 7.5-11$ & 32 & $3.953 \mid \infty$ \\
\hline optControl & $4759 \mid 1587 ; ; ; 1986$ & $36|-3.066894792|-3.066894792$ & $1.1-7|2.2-10| 8.8-11 \mid 1.3-10$ & 06 & 4.93 \\
\hline
\end{tabular}


Table 1: Performance of sdpt3.m. In the table, err = [pinfeas, dinfeas,relgap,relgap2], where relgap2 is the same as relgap but with the numerator replaced by $\left|\langle c, x\rangle-b^{T} y\right|$, and normXZ $=\max \left\{\left\|x^{*}\right\|,\left\|z^{*}\right\|\right\}$. We declare that $g_{P}\left(g_{D}\right)$ is $\infty$ if the computed number is larger than $10^{12}$.

\begin{tabular}{|c|c|c|c|c|c|c|}
\hline problem & $m \mid n_{s} ; n_{q} ; n_{l} ; n_{u}$ & & it. | primal obj | dual obj & err & time & $g_{P}\left|g_{D}\right|$ normXZ \\
\hline randomUnco & $264 \mid 130 ; ; ;$ & $17 \mid$ & 6.33323683-4| 6.33323351-4 & $2.7-14|1.0-12| 3.4-10 \mid 3.3-10$ & 01 & $2.142|1.882| 4.20$ \\
\hline randomCons & $650 \mid 298 ; ; ;$ & $17 \mid$ & $3.945591820 \mid 3.945591800$ & $2.2-12|1.0-12| 2.1-9 \mid 2.1-9$ & 01 & $4.272|9.573| 2.71$ \\
\hline randomwith & $430 \mid 130 ; ; ; 395$ & $22 \mid$ & $3.13274316-4 \mid 3.13274418-4$ & $7.5-13|3.8-10| 1.6-11 \mid 1.0-10$ & 01 & $9.992 \mid \infty$ \\
\hline
\end{tabular}

